\title{
Time-resolved optical conductivity and Higgs oscillations in two-band dirty superconductors
}

\author{
Rafael Haenel $\odot,{ }^{1,2,3}$ Paul Froese $\odot,{ }^{1,2}$ Dirk Manske, ${ }^{1}$ and Lukas Schwarz $\odot^{1}$ \\ ${ }^{1}$ Max Planck Institute for Solid State Research, 70569 Stuttgart, Germany \\ ${ }^{2}$ Department of Physics and Astronomy, University of British Columbia, Vancouver V6T 1Z4, Canada \\ ${ }^{3}$ Quantum Matter Institute, University of British Columbia, Vancouver V6T 1Z4, Canada
}

(Received 1 February 2021; revised 9 June 2021; accepted 14 September 2021; published 5 October 2021)

\begin{abstract}
Recent studies have emphasized the importance of impurity scattering for the optical Higgs response of superconductors. In the dirty limit, an additional paramagnetic coupling of light to superconducting condensate arises, which drastically enhances excitation. So far, most work concentrated on the periodic driving with light, where the third-harmonic generation (THG) response of the Higgs mode was shown to be enhanced. In this paper, we extend this analysis by calculating full temperature and frequency dependence of THG to better compare the theory with current experimental setups. We additionally calculate the time-resolved optical conductivity of single- and two-band superconductors in a two-pulse quench-probe setup, where we find good agreement with existing experimental results. We use the Mattis-Bardeen approach to incorporate impurity scattering and calculate explicitly the time-evolution of the system. In contrast to previous work we calculate the response not only within a time-dependent density-matrix formalism but also in a diagrammatic picture derived from an effective action formalism, which gives a deeper insight into the microscopic processes.
\end{abstract}

DOI: 10.1103/PhysRevB.104.134504

\section{INTRODUCTION}

When a continuous symmetry is spontaneously broken, collective excitations emerge. In the case of a superconductor, where the complex order parameter $\Delta e^{i \theta}$ acquires a finite value below a critical temperature $T_{C}$, two bosonic modes appear: the massive Higgs mode and a massless Goldstone mode [1,2]. They may be seen as amplitude $\delta \Delta$ and phase $\delta \theta$ fluctuations of the complex order parameter in the Mexican hat-shaped free-energy potential. When coupled to a gauge field, the Goldstone mode is shifted to the plasma frequency by means of the Anderson-Higgs mechanism while the Higgs mode remains a stable gapped excitation in the Terahertz regime [3].

In a two-band superconductor, two gapped Higgs modes and two phase modes exist. While the global phase fluctuation occurs again only at energies close to the plasma frequency for a charged condensate, the relative phase fluctuation, quantized as the Leggett mode, persists as a gapped excitation at low energies [4].

Experimental observation of Higgs and Leggett collective modes is difficult. Since these fields are scalar quantities, no linear coupling to the electromagnetic field exists at zero momentum [2]. Thus, there are no direct experimental signatures in linear response. As a consequence, experiments need to

Published by the American Physical Society under the terms of the Creative Commons Attribution 4.0 International license. Further distribution of this work must maintain attribution to the author(s) and the published article's title, journal citation, and DOI. Open access publication funded by the Max Planck Society. be performed in the nonlinear regime. Here, the challenge is twofold: Intense light sources are required but experiments also have to be performed on energy scales mostly within the superconducting gap such that optical excitation of quasiparticles does not deplete the condensate.

Recent developments in ultrafast Terahertz spectroscopy have caused a surge in interest to study collective excitations in non-equilibrium superconductors both in theory [5-10] and experiment [11-18], where first experimental signatures of the Higgs mode have been observed in various materials. The main excitation schemes so far consist of two approaches. First, samples are illuminated in a pump-probe setup where an excitation of the Higgs mode by a single-cycle $\mathrm{THz}$ pump acting as a quench appears as an oscillation of the probe signal as a function of pump-probe delay [11]. In a second type of experiment, the Higgs mode is resonantly driven by an intense multicycle pulse that yields an electrical field component of three times the pump frequency in the reflected or transmitted beam $[12,15,17]$.

The fact that characteristics of the Higgs mode in superconductors are observable in experiments is not self-evident. Early theoretical calculations in the clean limit predicted extremely weak experimental signatures that relied on breaking of the particle-hole symmetry. Therefore, the first observations [12] of the third-harmonic response generated by the Higgs mode was doubted [19] as it should be overlaid by much stronger charge fluctuations. Only recently, the role of impurities has been appreciated as it drastically enhances the coupling of light to the Higgs mode due to an additional paramagnetic coupling absent in the clean limit [20-23]. This coupling becomes the dominant contribution even for small disorder. It was further shown that impurity scattering yields qualitatively different 
behavior in the polarization dependence of the driving pulses [23].

Previous studies on third-harmonic generation with impurities concentrated mostly on the frequency dependence for fixed temperature [20-23] or temperature dependence for fixed frequency [21]. Here, we extend the analysis and calculate the full $2 \mathrm{~d}$ temperature- and frequency-dependent THG signal, which allows to better understand and compare the theory with current experimental setups where only the temperature can be sweeped for fixed driving frequency. We also calculate the individual contributions of density fluctuations, Leggett mode and Higgs mode to the third-harmonic generation response. Our results support the findings of a recent paper, where the third-harmonic response in the two-band superconductor $\mathrm{MgB}_{2}$ shows a resonance only for the lower gap [17]. This can be understood from the fact that the upper band is either in the clean limit or that the Fermi surface is very small.

In addition to the investigations of previous work on the effect of impurities in the periodic driving scheme, in this paper, we also explore the excitation with a two-pulse quench-probe scheme. We consider both one- and two-band superconductors where the bands can be in different impurity regimes. To calculate the time-resolved optical conductivity, we extend the density-matrix approach of [20] to a two-pulse excitation scheme. Here, the short first pulse acts as a quench, while the second probe pulse with variable time-delay probes the dynamics of the system.

We incorporate the effect of impurities in our model using the Mattis-Bardeen approximation [24]. This approach constitutes an excellent description for many conventional superconductors at least in the linear regime [25]. It has also been used in [20] but differs from the self-consistent Born approximation in [21] and [22].

Compared to the density-matrix approach in [20], we additionally consider a diagrammatic approach derived from an effective action formalism, where the Mattis-Bardeen ansatz is incorporated by an effective finite momentum interaction vertex. We show that this diagrammatic approach is equivalent to the density-matrix formalism. There are several advantages of this approach. First of all, it allows to understand the involved processes in more detail due to a disentangling with individual diagrams. Secondly, as the Matsubara sums in the expression can be analytically evaluated, this approach is computationally more efficient and allows calculations with more accuracy and greater variation of parameters as a full $2 \mathrm{~d}$ temperature and frequency sweep. And finally, in the diagrammatic approach it is natural to include Coulomb interaction to perform a fully gauge-invariant calculation.

This article is organized as follows. In Sec. II we formulate the model (a) in terms of a diagrammatic expansion of an effective action and (b) in terms of a density-matrix equation of motion approach that was previously established by Murotani and Shimano [20]. The two formulations are equivalent. We then discuss results in the case of a single-band superconductor in a pump-probe scenario in Sec. III. In Sec. IV we study in detail the case of a two-band superconductor motivated by material parameters of $\mathrm{MgB}_{2}$. Here we focus on both pumpprobe and third-harmonic generation (THG) experiments. We summarize all results in Sec. V.

\section{MODEL}

\section{A. Hamiltonian}

We consider the BCS multiband Hamiltonian

$$
\mathcal{H}_{0}=\sum_{i \mathbf{k} \sigma} \epsilon_{i \mathbf{k}} c_{i \mathbf{k} \sigma}^{\dagger} c_{i \mathbf{k} \sigma}-\sum_{i j \mathbf{k} \mathbf{k}^{\prime}} U_{i j} c_{i \mathbf{k} \uparrow}^{\dagger} c_{i-\mathbf{k} \downarrow}^{\dagger} c_{j-\mathbf{k}^{\prime} \downarrow} c_{j \mathbf{k}^{\prime} \uparrow},
$$

where $\epsilon_{i \mathbf{k}}=s_{i}\left(\mathbf{k}^{2} / 2 m_{i}-\epsilon_{F_{i}}\right)$ is the parabolic dispersion of the $i$ th band with Fermi-energy $\epsilon_{F_{i}}$ and electron mass $m_{i}$. The factor $s_{i}= \pm$ determines electron- or hole-like character of the respective band.

At the mean-field level the interacting term is decoupled in the pairing channel,

$$
\sum_{i \mathbf{k}} \Delta_{i} c_{i-\mathbf{k} \uparrow}^{\dagger} c_{i \mathbf{k} \downarrow}^{\dagger}+\text { H.c. }
$$

where order parameters $\Delta_{i}$ are self-consistently determined by the BCS gap equation $\Delta_{i}=\sum_{j \mathbf{k}} U_{i j}\left\langle c_{j-\mathbf{k} \downarrow} c_{j \mathbf{k} \uparrow}\right\rangle$ [26]. The order parameters of different bands are mixed by off-diagonal terms in the coupling matrix $U_{i j}$. In the present paper, we parametrize gap-mixing by a parameter $v$ and define

$$
U_{i j}=\left(\begin{array}{cc}
U_{11} & v U_{11} \\
v U_{11} & U_{22}
\end{array}\right)
$$

For given $\Delta_{i}$ and $v$ we can find $U_{11}$ and $U_{22}$ such that the gap equation is satisfied.

To model an experimental probe with a laser pulse, we introduce a time-dependent vector potential $\mathbf{A}(t)=A(t) \mathbf{e}$ with polarization vector e by means of minimal coupling,

$$
\mathcal{H}_{1}=-\sum_{i \mathbf{k}^{\prime} \sigma} \mathbf{J}_{i \mathbf{k k}^{\prime}} \cdot \mathbf{A} c_{i \mathbf{k} \sigma}^{\dagger} c_{i \mathbf{k}^{\prime} \sigma}+\sum_{i \mathbf{k} \sigma} \frac{s_{i} e^{2}}{2 m_{i}} \mathbf{A}^{2} c_{i \mathbf{k} \sigma}^{\dagger} c_{i \mathbf{k} \sigma},
$$

where $J_{i \mathbf{k} \mathbf{k}^{\prime}}=\left\langle i \mathbf{k}\left|\frac{e \mathbf{p}_{i}}{m_{i}}\right| i \mathbf{k}^{\prime}\right\rangle$ are intraband transition matrix elements of the current operator. Here, we have neglected interband excitation, which for materials like $\mathrm{MgB}_{2}$ is strongly suppressed by the separation of Fermi surfaces in the Brillouin zone. The two terms in $\mathcal{H}_{1}$ corresponds to the paramagnetic and diamagnetic coupling of the laser field, respectively. The full Hamiltonian is given by $\mathcal{H}=\mathcal{H}_{0}+\mathcal{H}_{1}$.

\section{B. Impurity scattering}

In a clean system momentum conservation yields $\mathbf{J}_{i \mathbf{k} \mathbf{k}^{\prime}} \sim$ $\delta_{\mathbf{k k}^{\prime}}$, or $\mathbf{J}_{i \mathbf{k} \mathbf{k}^{\prime}} \sim \delta_{\mathbf{k}, \mathbf{k}^{\prime} \pm \mathbf{q}}$ if a photon wave vector $\mathbf{q}$ is considered. In disordered systems, translational invariance is broken, so that transitions between states of different momenta are allowed. Here, we adopt the approach of Murotani and Shimano [20] and model the effects of impurities within the Mattis-Bardeen (MB) approximation [24]. Explicitly, impurities enter through the approximation

$$
\begin{aligned}
\left\langle\left|\mathbf{e} \cdot \mathbf{J}_{i \mathbf{k} \mathbf{k}^{\prime}}\right|^{2}\right\rangle_{\mathrm{Av}} & =\int \frac{d \Omega_{\mathbf{k}}}{4 \pi} \frac{d \Omega_{\mathbf{k}^{\prime}}}{4 \pi}\left|\mathbf{e} \cdot \mathbf{J}_{i \mathbf{k} \mathbf{k}^{\prime}}\right|^{2} \\
& \approx \frac{\left(e v_{F_{i}}\right)^{2}}{3 N_{i}(0)} W\left(\epsilon_{i \mathbf{k}}, \epsilon_{i \mathbf{k}^{\prime}}\right), \\
W\left(\epsilon_{i \mathbf{k}}, \epsilon_{i \mathbf{k}^{\prime}}\right) & =\frac{1}{\pi} \frac{\gamma_{i}}{\left(\epsilon_{i|\mathbf{k}|}-\epsilon_{i\left|\mathbf{k}^{\prime}\right|}\right)^{2}+\gamma_{i}^{2}}
\end{aligned}
$$


with Fermi velocity $v_{F_{i}}$, density of states at the Fermi level $N_{i}(0)$, and impurity scattering rate $\gamma_{i}$. A derivation of this matrix element is given in Ref. [20].

The Mattis-Bardeen approximation is applicable in the regime $\gamma_{i} \ll \epsilon_{F_{i}}$ for isotropic impurity scattering, and uniform charge density [24]. These conditions are met for the superconductors considered in the present paper, where the dirty limit is already achieved when the impurity concentration $\gamma$ is of the order of the superconducting gap $\Delta$.

We see that impurity scattering broadens the $\delta_{\mathbf{k k}^{\prime}}$ distribution into a Lorentzian of width $\gamma_{i}$ centered at zero momentum transfer. The bandstructure defined by $\mathcal{H}_{0}$ remains unaffected in this approximation. Instead of broadening the momentum resolution of the bandstructure, one may view impurities as effectively broadening the momentum of the photon.

While the simplicity of the Mattis-Bardeen approach is appealing, more realistic models of impurities rely on exact numerical treatment [23] or diagrammatic summation of impurity ladder corrections [21,22].

\section{Effective action}

We first present a perturbative solution of above Hamiltonian by a path-integral formalism in imaginary time $\tau$ [27-30]. The full problem is formally captured by the partition function $\mathcal{Z}=\int \mathcal{D}\left(c^{\dagger} c\right) e^{-S}$ with the euclidean action

$$
S=\int_{0}^{\beta} d \tau\left(\sum_{i \mathbf{k} \sigma} c_{i \mathbf{k} \sigma}^{\dagger} \partial_{\tau} c_{i \mathbf{k} \sigma}+\mathcal{H}\right) .
$$

As detailed in Appendix A, we decouple the interacting part of $\mathcal{H}$ in the paring channel, introducing collective fields $\Delta_{i}\left(\omega_{n}\right) \exp \left(i \theta_{i}\left(\omega_{n}\right)\right) . \Delta_{i}$ and $\theta_{i}$ describe amplitude and phase fluctuations, respectively, of the superconducting condensate. These collective fluctuations are dependent on time only, i.e. the Hamiltonian supports only $\mathbf{k}=0$ excitations of Higgs and phase fields.

Performing the fermionic path integral results in an effective action $S\left[\Delta_{i}, \theta_{i}, \mathbf{A}\right]$ in bosonic and classical EM fields [see Eq. (A13)], where now $\mathcal{Z}=\int \prod_{i} \mathcal{D} \Delta_{i} \mathcal{D}_{i} \theta_{i} e^{-S\left[\Delta_{i}, \theta_{i}, \mathbf{A}\right]}$. We only keep terms quadratic in collective fields $\Delta_{i}, \theta_{i}$ and to fourth order in $\mathbf{A}$. The resulting terms are diagrammatically presented in Fig. 1 and Fig. 2 and their integral expressions are derived in Appendix A.

The diagrammatic representation contains Higgs fields $\Delta_{i}(\omega)$ (blue-dashed lines), phase fields (green-dotted lines), and EM fields (wavy lines). Paramagnetic coupling to the photon field corresponds to vertices with a single photon field line, implying the factor $A(\omega)$. Diamagnetic vertices with two photon field lines contribute the term $A^{2}(\omega)=$ $\int d \omega^{\prime} A\left(\omega-\omega^{\prime}\right) A\left(\omega^{\prime}\right)$. Numeric labels $(0,1,3)$ indicate Pauli matrices $\sigma_{0}, \sigma_{1}, \sigma_{3}$ in Nambu space where $\sigma_{0}$ is the identity. Only paramagnetic vertices introduce external momentum. Solid black lines correspond to mean-field Nambu Green's functions

$$
G_{0, i}=\left[i \omega_{n}-\epsilon_{i \mathbf{k}} \sigma_{3}+\Delta_{i} \sigma_{1}\right]^{-1}
$$

and loops imply a trace over Nambu indices, frequencies, and momenta. (a)

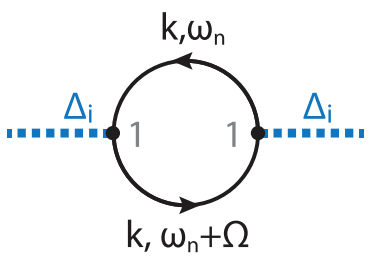

(c)

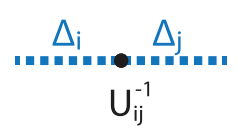

(e)

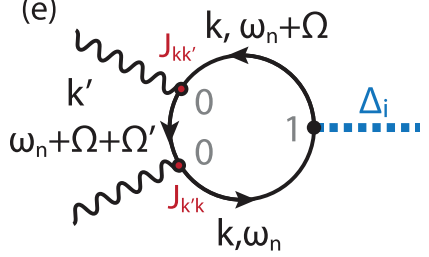

(b)

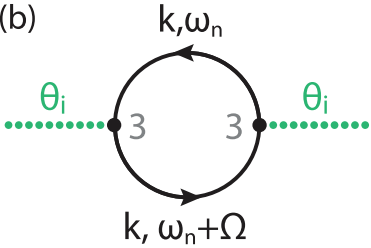

(d)

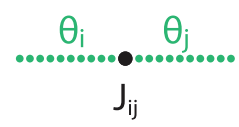

(f)

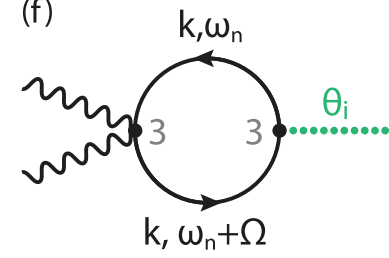

FIG. 1. Diagrammatic representation of terms in the quadratic effective action $S\left[\Delta_{i}, \theta_{i}, \boldsymbol{A}\right]$ in Eq. (A13) involving Higgs fields (left column) and phase fields (right column). Bubbles correspond to susceptibilities listed in Eqs. (A23)-(A27). The blue (green) dotted lines represent Higgs (Leggett) propagators, the wavy black line represents the electromagnetic field and the solid black line the Nambu Greens function. Numbers $0,1,3$ at the vertices represent Pauli matrices $\sigma_{0}, \sigma_{1}, \sigma_{3}$ acting in Nambu space. [(a),(b)] Higgs and phase susceptibilities $\chi_{i}^{\sigma_{1} \sigma_{1}}, \chi_{i}^{\sigma_{3} \sigma_{3}}$. (c) Coupling of Higgs modes where vertex is the inverse of Eq. (3). (d) Josephson coupling of phase modes responsible for Leggett mode. The coupling matrix $J$ is defined in Eq. (A17). (e) Paramagnetic coupling of Higgs modes with susceptibility $\chi^{\sigma_{0} \sigma_{0} \sigma_{1}}$. (f) Diamagnetic coupling of phase modes with $\chi^{\sigma_{3} \sigma_{3}}$. Other couplings at Gaussian level vanish in the presence of particle-hole symmetry. (a)

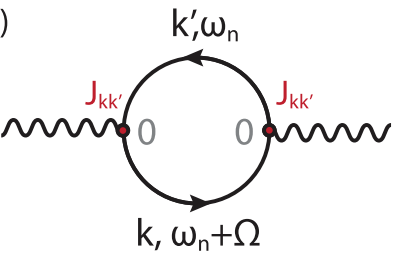

(c)

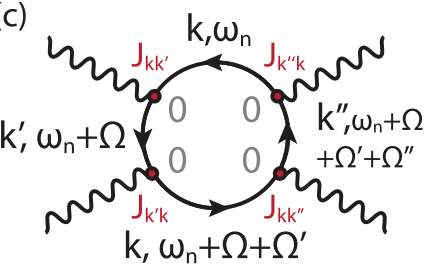

(b)

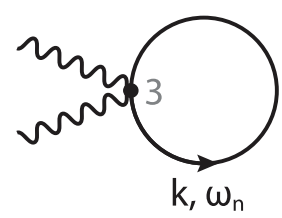

(d)

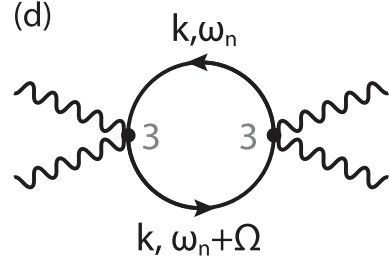

FIG. 2. Diagrammatic representation of density-fluctuation contributions in the effective action $S\left[\Delta_{i}, \theta_{i}, \mathbf{A}\right]$ Eq. (A13). Paramagnetic (a) and diamagnetic (b) terms defining the linear response current $\left.\mathbf{j}\right|_{1}$. The paramagnetic contribution (a) vanishes in the clean limit. Paramagnetic (c) and diamagnetic (d) terms contributing to nonlinear current $\left.\mathbf{j}\right|_{3}$. 
(a)

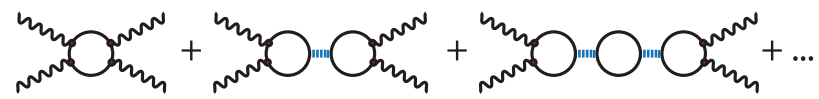

(b)

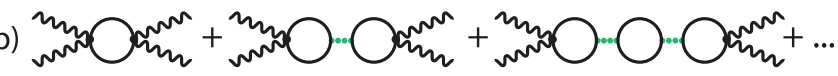

FIG. 3. RPA summation of collective fields in the effective action. (a) Higgs modes renormalize paramagnetic nonlinear current. Here, blue dashed lines correspond to the coupling matrix $U / 2$. (b) Phase modes renormalize diamagnetic current. Green dotted lines denote coupling matrix $J^{-1}$.

Figures 1 and 2 are a complete representation of all terms in the quadratic action in the presence of particle-hole symmetry and impurities in the MB approximation.

In the clean limit paramagnetic photon lines no longer carry momentum and, as a consequence, diagrams 1(e) and 2(a) vanish. The inclusion of paramagnetic diagrams with vertices $J_{i \mathbf{k} \mathbf{k}^{\prime}}$ determined by the MB model is the main difference of the diagramatic formalism from other literature [19,31].

Absence of diagram 1(e) in the clean limit implies that the Higgs mode does not couple to light without impurities. However, when a non-parabolicity of the bandstructure is taken into account, a diamagnetic coupling to the Higgs mode arises, yielding an additional, nonvanishing diagram $[7,19,20]$.

We note that paramagnetic and diamagnetic terms do not mix in the present model. Consequently, the partition function factors into two contributions $\mathcal{Z}=\mathcal{Z}_{\text {para }} \mathcal{Z}_{\text {dia }}$. Since only the paramagnetic part is affected by impurities, and since $\mathcal{Z}_{\text {para }}$ does not contain phase contributions, we conclude that only the Higgs mode and density fluctuations are sensitive to impurity scattering in the MB approximation.

The path integrals over $\Delta_{i}, \theta_{i}$ can be performed exactly at Gaussian level. This is equivalent to an RPA renormalization of the density fluctuation terms diagrammatically represented in Fig. 3 where the dashed and dotted lines correspond to coupling matrices $U_{i j} / 2$ and Josephson coupling matrices $J_{i j}^{-1}$, respectively. After Gaussian integration, one is left with $S[A(\omega)]$, explicitly given in Eq. (A28). A functional derivative with respect to $A(\omega)$ gives the current

$$
j(\omega)=-\frac{\delta S[A(\omega)]}{\delta A(\omega)} .
$$

In the single-band case, the diamagnetic RPA series Fig. 3(b) is exactly zero. This results from the cancellation of the diamagnetic density-fluctuation contribution 2(d) with the phase mode contribution, shown in Eq. (A31) of Appendix A. The cancellation is exact in the continuum limit and does not rely on particle-hole symmetry. For a lattice model, the cancellation is imperfect away from low or large filling. At half filling, where particle-hole symmetry is exact, the phase mode contribution vanishes and the diamagnetic current remains finite [19], hence no cancellation occurs.

In the two-band case, the phase supports both a Goldstone mode and the Leggett mode. Here, the Goldstone contribution cancels the diamagnetic third-order current, while the Leggett mode is the remaining source of the total diamagnetic current.

The present results are insensitive to long-range Coulomb interaction. We show in Appendix B how, in the presence of particle-hole symmetry, the same expression as in the uncharged case are derived for the continuum model.

\section{Density-matrix equations of motion}

We solve for the time dynamics of above Hamiltonian using a density-matrix approach. To this end, we define the density matrix $\rho=\left|\psi_{0}\right\rangle\left\langle\psi_{0}\right|$, or, in the basis of Bogoliubovde Gennes,

$$
\left(\begin{array}{c}
\psi_{i \mathbf{k}}^{1} \\
\psi_{i \mathbf{k}}^{2}
\end{array}\right)=\left(\begin{array}{cc}
u_{i \mathbf{k}} & -v_{i \mathbf{k}} \\
v_{i \mathbf{k}}^{*} & u_{i \mathbf{k}}
\end{array}\right)\left(\begin{array}{c}
c_{i \mathbf{k} \uparrow} \\
c_{i(-\mathbf{k}) \downarrow}^{\dagger}
\end{array}\right),
$$

we have

$$
\rho=\left(\begin{array}{cc}
\rho_{i \mathbf{k} \mathbf{k}^{\prime}}^{11} & \rho_{i \mathbf{k} \mathbf{k}^{\prime}}^{12} \\
\rho_{i \mathbf{k} \mathbf{k}^{\prime}}^{21} & \rho_{i \mathbf{k} \mathbf{k}^{\prime}}^{22}
\end{array}\right)=\left(\begin{array}{cc}
\left\langle\psi_{i \mathbf{k}}^{1 \dagger} \psi_{i \mathbf{k}^{\prime}}^{1}\right\rangle & \left\langle\psi_{i \mathbf{k}}^{1 \dagger} \psi_{i \mathbf{k}^{\prime}}^{2}\right\rangle \\
\left\langle\psi_{i \mathbf{k}}^{2 \dagger} \psi_{i \mathbf{k}^{\prime}}^{1}\right\rangle & \left\langle\psi_{i \mathbf{k}}^{2 \dagger} \psi_{i \mathbf{k}^{\prime}}^{2}\right\rangle
\end{array}\right) .
$$

The time dependence of $\rho$ is given by Heisenberg's equation of motion,

$$
i \partial_{t} \rho=[\rho, H],
$$

where $H$ is the operator $\mathcal{H}$ in the $\mathrm{BdG}$ basis.

We are interested in computing the dynamics of the current $\mathbf{j}=-\left\langle\frac{\delta \mathcal{H}}{\delta \mathbf{A}}\right\rangle=\mathbf{j}_{P}+\mathbf{j}_{D}$, consisting of a paramagnetic and diamagnetic contribution,

$$
\begin{gathered}
\mathbf{j}_{P}=\sum_{i \mathbf{k} \mathbf{k}^{\prime}} \mathbf{J}_{i \mathbf{k} \mathbf{k}^{\prime} \sigma}\left\langle c_{i \mathbf{k} \sigma}^{\dagger} c_{i \mathbf{k}^{\prime} \sigma}\right\rangle, \\
\mathbf{j}_{D}=-\sum_{i \mathbf{k} \sigma} \frac{s_{i} e^{2}}{m_{i}} \mathbf{A}\left\langle c_{i \mathbf{k} \sigma}^{\dagger} c_{i \mathbf{k} \sigma}\right\rangle,
\end{gathered}
$$

as well as the dynamics of the superconducting order parameter

$$
\Delta_{i}=\sum_{j \mathbf{k}} U_{i j}\left\langle c_{j(-\mathbf{k}) \downarrow} c_{j \mathbf{k} \uparrow}\right\rangle .
$$

To apply the MB substitution, we further expand above equations of motion in orders of $A(t)$. To account for effects of a THG response, we consider terms up to third order. As detailed in Appendix E, the current only has odd order components $\mathbf{j}=\left.\mathbf{j}\right|_{0}+\left.\mathbf{j}\right|_{3}+\ldots$ and the gap contains even contributions of $A, \Delta=\left.\Delta\right|_{0}+\left.\delta \Delta\right|_{2}+\ldots$.

Finally, we exploit the rotational invariance of our model and perform the integral over angular degrees of freedom explicitly. Thus, by replacing all momentum summations by an integral $\sum_{\mathbf{k}} \rightarrow N_{i}(0) \int d \epsilon_{i \mathbf{k}} \int \frac{d \Omega_{\mathbf{k}}}{4 \pi}$, we effectively reduce the model to a one-dimensional system. Note that rotational invariance of our continuum model neglects polarization dependence of observable quantities.

We are left to compute the equations of motion of the first-order expectation values, $\left.\rho_{i \mathbf{k} \mathbf{k}^{\prime}}\right|_{1}$, and the angle-averaged quantities

$$
\begin{aligned}
& R_{i}^{a b}\left(\epsilon_{i|\mathbf{k}|}, \epsilon_{i\left|\mathbf{k}^{\prime}\right|}\right)= \frac{1}{\int \frac{d \Omega_{\mathbf{k}}}{4 \pi} \frac{d \Omega_{\mathbf{k}^{\prime}}}{4 \pi}\left|\mathbf{J}_{i \mathbf{k} \mathbf{k}^{\prime}} \cdot \mathbf{e}\right|^{2}} \\
& \times\left.\int \frac{d \Omega_{\mathbf{k}}}{4 \pi} \frac{d \Omega_{\mathbf{k}^{\prime}}}{4 \pi} \mathbf{e} \cdot \mathbf{J}_{i \mathbf{k} \mathbf{k}^{\prime}} \rho_{i \mathbf{k} \mathbf{k}^{\prime}}^{a b}\right|_{3}, \\
& r_{i}^{a b}\left(\epsilon_{i|\mathbf{k}|}\right)=\left.\int \frac{d \Omega_{\mathbf{k}}}{4 \pi} \rho_{i \mathbf{k k}}^{a b}\right|_{2} .
\end{aligned}
$$


We solve them numerically using a Runge-Kutta solver on a discretized energy grid $\epsilon_{\left|\mathbf{k}_{i}\right|}$ of up to $10^{3}$ points in the interval $\left[-\omega_{D}, \omega_{D}\right]$. A detailed derivation and explicit presentation of the full equations of motion is given in Appendix E.

\section{SINGLE-BAND SUPERCONDUCTIVITY}

Motivated by the experiment of Matsunaga et al. [11] we choose parameters $\Delta=1.3 \mathrm{meV}, \epsilon_{F}=1 \mathrm{eV}, m=0.78 m_{e}$, $s=1$, and $\omega_{D}=20 \mathrm{meV}$ that reflect measurements and $a b$ initio calculations on $\mathrm{NbN}$ [32].

\section{A. Optical conductivity}

We begin by computing the optical conductivity in linear response,

$$
\sigma(\omega)=\frac{\left.j(\omega)\right|_{1}}{i \omega A(\omega)}
$$

This can be done in either of two ways. First, by implementing a time-dependent density-matrix simulation with pulse $A(t)$. The numerically evaluated current $\left.j(t)\right|_{1}$ and the pulse are then Fast-Fourier transformed and Eq. (18) is evaluated. Here, one needs to choose a pulse of sufficient $\omega$ bandwidth such that the region of interest is covered.

The second way involves the functional derivative of the diagrams in Figs. 2(a) and 2(b) according to Eq. (9). At $T=0$ one obtains the expression for the real part

$$
\begin{aligned}
\sigma^{\prime}(\omega)= & \frac{1}{i \omega} \frac{v_{F}^{2}}{3 N} \int d \epsilon d \epsilon^{\prime} W\left(\epsilon, \epsilon^{\prime}\right) \chi^{\prime \prime \sigma_{0} \sigma_{0}}\left(\epsilon, \epsilon^{\prime}, \omega\right) \\
= & \frac{1}{i \omega} \frac{v_{F}^{2} N}{3} \int d \epsilon d \epsilon^{\prime} W\left(\epsilon, \epsilon^{\prime}\right)\left(1-\frac{\epsilon \epsilon^{\prime}+\Delta^{2}}{E E^{\prime}}\right) \\
& \times \frac{E+E^{\prime}}{(w+i \eta)^{2}-\left(E+E^{\prime}\right)^{2}},
\end{aligned}
$$

where $E^{\prime}=\sqrt{\Delta^{2}+\epsilon^{\prime 2}}, W\left(\epsilon, \epsilon^{\prime}\right)$ is the Lorentzian of Eq. (6), $\mathrm{N}$ the density of states at the Fermi surface, and $\eta$ is an infinitesimal positive constant.

We can understand the analytical structure of $\sigma^{\prime}(\omega)$ by inspecting the susceptibility $\chi^{\prime \prime \sigma_{0} \sigma_{0}}\left(\epsilon, \epsilon^{\prime}, \omega\right)$. For $\omega<2 \Delta$ it vanishes exactly. For $\omega>2 \Delta$ its structure is exemplary shown in Fig. 4. We observe two straight spectral lines at $\epsilon^{\prime}=$ $\pm \omega+\epsilon$. These features can be understood in the picture of a particle-hole or hole-particle excitation process, illustrated in Fig. 4(a). $\chi^{\prime \prime \sigma_{0} \sigma_{0}}\left(\epsilon, \epsilon^{\prime}, \omega\right)$ has nonzero spectral weight at given $\epsilon, \epsilon^{\prime}$ if an occupied state at $\epsilon$ can be excited into a state at $\epsilon^{\prime}$ by a photon of frequency $\omega$. Multiplication of the integrand in Eq. (19) with $W\left(\epsilon-\epsilon^{\prime}\right)$ enforces momentum conservation.

In this picture it is easy to see that the total spectral weight $\chi^{\prime \sigma_{0} \sigma_{0}}(\omega)=\int d \epsilon d \epsilon^{\prime} \chi^{\prime \sigma_{0} \sigma_{0}}\left(\epsilon, \epsilon^{\prime}, \omega\right)$ should be approximately proportional to $\Theta(\omega-2 \Delta)(\omega-2 \Delta)$, where $\Theta$ is the Heaviside function. Since $W\left(\epsilon-\epsilon^{\prime}\right)$ is constant along contours $\epsilon^{\prime}= \pm \omega+\epsilon$, we find the simple analytical approximation

$$
\sigma^{\prime}(\omega) \propto \Theta(\omega-2 \Delta)(\omega-2 \Delta) \frac{\gamma}{\omega^{2}+\gamma^{2}}
$$

that holds for $\omega \gg 2 \Delta$ in the dirty limit $\gamma \gg \Delta$.

In Fig. 5 we plot numerically evaluated real and imaginary parts $\sigma^{\prime}(\omega), \sigma^{\prime \prime}(\omega)$ of the optical conductivity for various
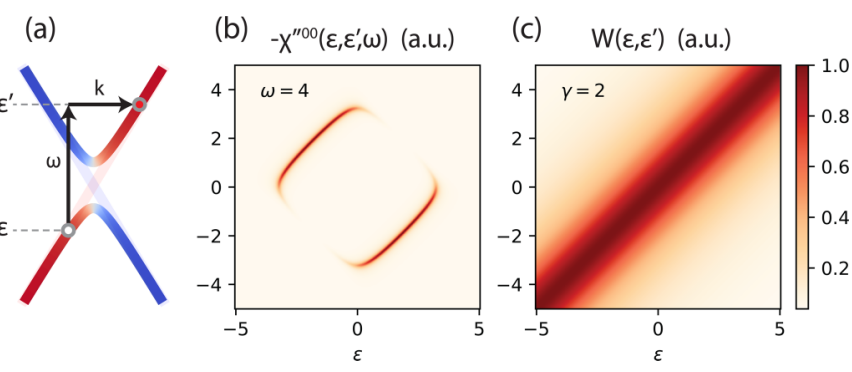

FIG. 4. (a) Schematic of a particle-hole excitation process where the pulse contributes energy $\omega$ and momentum $k$. Red (blue) colors indicate electron (hole) character. (b) The susceptibility $-\chi^{\prime \prime \sigma_{0} \sigma_{0}}$ has finite weight only for $\epsilon, \epsilon^{\prime}$ corresponding to valid state in an excitation process with $\omega=4$. Rounded features are a result of the gap $2 \Delta$. For $\omega<2 \Delta, \chi^{\prime \prime \sigma_{0} \sigma_{0}}$ is identically zero since no optical excitation is not possible. (c) Momentum conservation is enforced by the factor $W\left(\epsilon-\epsilon^{\prime}\right)$ in Eq. (19).

impurity concentrations and temperatures. $\sigma^{\prime}$ shows a clear conductivity gap below $2 \Delta$. In the clean limit, a pronounced coherence peak is observed around $2 \Delta$, reflecting the additional density of states amassed above the quasiparticle gap. The conductivity peak grows and shifts to higher $\omega$ as $\gamma$ is increased. It then broadens into the characteristic dome shape frequently observed in experiment $[11,24,33]$. In the $T \rightarrow 0$ limit, the conductivity is expected to show a condensate $\delta$ peak at $\omega=0$, which is not numerically resolvable. Instead, we observe a buildup of spectral weight around $\omega=0$ as the condensate peak is broadened at finite temperatures. The imaginary part $\sigma^{\prime \prime}$ follows a $1 / \omega$ power law as expected for a superconducting state.

The linear response optical conductivity contains information of the bandstructure only and is unaffected by collective
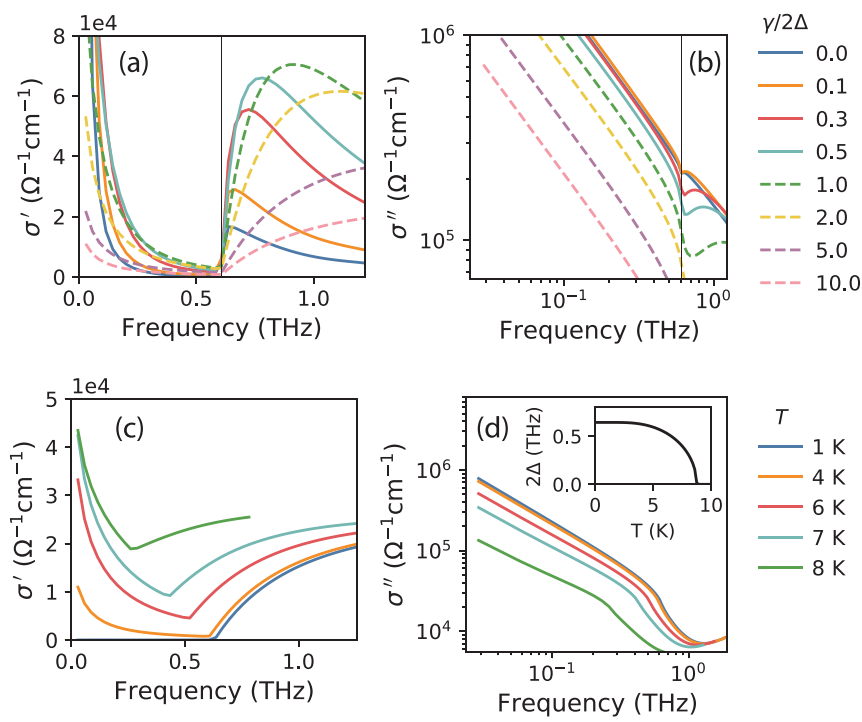

FIG. 5. Real part $\sigma^{\prime}$ and imaginary part $\sigma^{\prime \prime}$ of the optical conductivity to first order in the vector potential $A$. [(a),(b)] Impurity scattering rates dependence for fixed temperature $T=4 \mathrm{~K}$. [(c),(d)] Temperature dependence for fixed scattering rate $\gamma / 2 \Delta=10 . \sigma^{\prime}$ shows a characteristic conductivity gap below $T_{C}$ and both $\sigma^{\prime}, \sigma^{\prime \prime}$ diverge in the static limit. The inset in (d) shows the temperature dependence of the gap. 

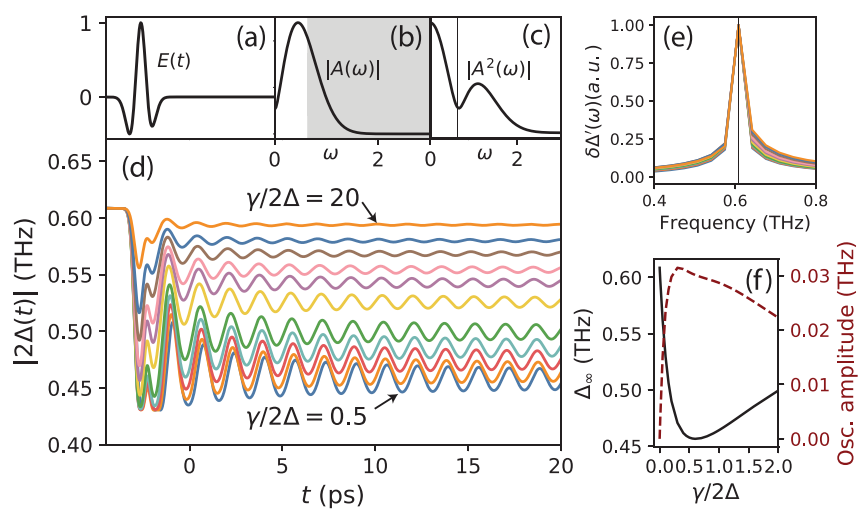

FIG. 6. (a) Pulse field $E(t)$ realizing a quench. (b) Spectral composition $|A(\omega)|$. The gray shaded area illustrates the quasi-particle continuum. (c) Spectral composition $\left|A^{2}(\omega)\right|=$ $\left|\int d \omega^{\prime} A\left(\omega-\omega^{\prime}\right) A\left(\omega^{\prime}\right)\right|$ of the second-order component $A^{2}(t)$ responsible for excitation of collective modes. The peak around zero frequency corresponds to a DFG process while the peak at finite 1.2 $\mathrm{THz}$ is a SFG process. (d) Evolution of the magnitude of the order parameter $|2 \Delta(t)|$ for impurity strength varying from $\gamma / 2 \Delta=$ 0.5 to 20 and Fourier spectrum of the gap oscillations (e). (f) Relaxation value $\Delta_{\infty}$ and amplitude of oscillation show a very similar dependence as a function of disorder strength, which has maximum effect at around $\gamma \approx \Delta$.

modes. This can be inferred from the diagrammatic description where all terms in the RPA renormalization of diagram Fig. 2(a) containing $\mathbf{k}=0$ collective fluctuations vanish exactly. To reveal the presence of collective modes, we turn to the dynamics of the superconducting order parameter and the nonlinear current $\left.j\right|_{3}$ and additionally model realistic $\mathrm{THz}$ pulses in a pump-probe setting.

\section{B. Excitation of Higgs mode}

We choose the electromagnetic pulse form $A(t)=$ $A_{0} \exp \left(-\left(t-t^{\prime}\right)^{2} / 2 \tau^{2}\right) \cos \Omega t$ with coefficients to match the reported data of Ref. [11]. The resulting waveform is shown in Fig. 6(a).

A characteristic property of a pump pulse is its pulse length $\tau$ compared to the natural timescale of the superconductor $1 / \Delta$. For $\tau \ll 1 / \Delta$ the superconductor is quenched, while it is adiabatically driven in the opposite limit of $\tau \gg 1 / \Delta$.

The different behavior in the two limits can be intuitively understood within the diagrammatic picture. Here, the pulse induced change of the order parameter $\delta \Delta(\omega)$ is given by the (a)

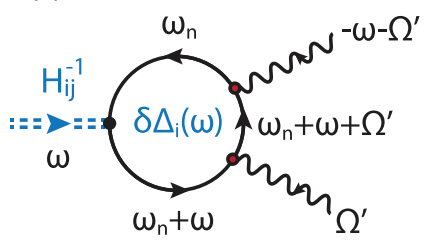

(b)

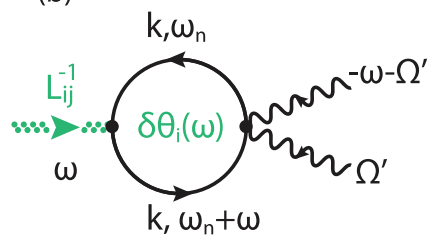

FIG. 7. Diagrammatic representation of (a) $\delta \Delta_{i}(\omega)$ and (b) $\delta \theta_{i}(\omega)$. Double lines correspond to the RPA summation of Fig. 3. diagram in Fig. 7(a), which has the integral expression

$$
\begin{aligned}
\delta \Delta(\omega)= & \frac{1}{2} \int d \omega^{\prime} \sum_{\mathbf{k} \mathbf{k}^{\prime}}\left|J_{\mathbf{k} \mathbf{k}^{\prime}}\right| \frac{\chi^{\sigma_{0} \sigma_{0} \sigma_{1}}\left(\omega, \omega^{\prime}, \mathbf{k}, \mathbf{k}^{\prime}\right)}{\chi^{\sigma_{1} \sigma_{1}}(\omega)+2 / U} \\
& \times A\left(\omega^{\prime}\right) A\left(-\omega-\omega^{\prime}\right) .
\end{aligned}
$$

Presence of a collective Higgs mode translates into a peak of the kernel $K(\omega)=\left(\chi^{\sigma_{1} \sigma_{1}}(\omega)+2 / U\right)^{-1}$ at the characteristic mode energy $\omega_{H}=2 \Delta$. Excitation of the collective mode, however, is only possible if energy conservation is satisfied, i.e., if $A\left(\omega^{\prime}\right) A\left(-\omega_{H}-\omega^{\prime}\right)$ is finite for some $\omega^{\prime}$. Higgs oscillations are therefore expected when the Fourier transform of the squared vector potential $A^{2}(\omega)=\int d \omega^{\prime} A\left(\omega-\omega^{\prime}\right) A\left(\omega^{\prime}\right)$ overlaps with the mode-energy $\omega_{H}$. The double-peaked structure of $A(\omega)$ is shown in Fig. 6(c). The first peak, centered at $\omega=0$, corresponds to a difference frequency generation process (DFG), while the second peak at $\omega=2 \Omega$ corresponds to a sum frequency generation process (SFG). The resonance frequency of the Higgs mode $\omega_{H}$ is illustrated by a vertical line. Remaining terms in Eq. (21) describe the coupling to light in presence of impurities and ensure momentum conservation in a virtual two step excitation process.

Let us now consider two limiting cases of the optical pulse width. For $\Delta \tau \ll 1$, the frequency spectrum of $A^{2}(\omega)$ is very broad. The response of $\delta \Delta(\omega)$ is then dominated by the sharp resonance peak of $K(\omega)$ giving rise to pronounced $2 \Delta$-oscillations of the superconducting gap in the time domain. Since the DFG peak is guaranteed to overlap with the Higgs resonance, these oscillations will always be present, independent of the frequency of the optical pulse. The SFG process only contributes if the pulse frequency lies in the vicinity of $\Omega \approx \Delta$.

In the transient limit, $\Delta \tau \gg 1$, the spectrum of $\delta \Delta(\omega)$ is finite only for a narrow region around $2 \Omega$. In the time domain, the gap shows forced $2 \Omega$ oscillations, which are resonantly enhanced for $2 \Omega \approx 2 \Delta$.

Following Matsunaga [11], we choose a pulse with $\Delta \tau=$ 0.68 , closest to the quench scenario, and perform simulations within the density-matrix formulation. The order parameter responds to the $\mathrm{THz}$ pulse by a marked drop followed by damped oscillations around a new asymptotic value $\Delta_{\infty}=\Delta(t \rightarrow \infty)$ of frequency $2 \Delta=0.6 \mathrm{THz}$ as displayed in Figs. 6(d) and 6(e). The drop of the equilibrium gap is captured by the $\omega=0$ component of $\delta \Delta$. Evaluating Eq. (21) for $\omega=0$, one finds that $\chi^{\sigma_{0} \sigma_{0} \sigma_{1}}\left(\omega=0, \omega^{\prime}, \mathbf{k}, \mathbf{k}^{\prime}\right)$ is finite only for $\omega^{\prime}>2 \Delta$, similar to the discussion in Sec. III A. Consequently, $\delta \Delta(0)$ is nonzero only if $|A(\omega)|^{2}$ overlaps with the quasiparticle continuum, which is illustrated in Fig. 6(b). In physical terms, depletion of the superconducting order parameter is a consequence of quasiparticle excitation by $A(\omega)$.

Both the oscillation amplitude and $\Delta_{\infty}$ show a strong dependence on the impurity scattering rate and are peaked at $\gamma \approx \Delta$ as shown in Fig. 6(f). This is a consequence of momentum conservation. For $\gamma \rightarrow 0$, Higgs oscillations vanish exactly.

We note that order parameter dynamics are expected to show oscillations of frequency $2 \Delta_{\infty}$ and not, as in our case, $2 \Delta(t=0)[6,34] .2 \Delta_{\infty}$ oscillations have also been observed in experiment [11]. The discrepancy can be attributed to the expansion in powers of the pump field $A(t)$ performed in the 
time-dependent density-matrix formalism. If contributions to $\delta \Delta$ beyond the second order are considered, the oscillation frequency of the order parameter should correctly reflect the non-equilibrium value $2 \Delta_{\infty}$.

From the diagrammatic point of view this discrepancy arises since calculations are performed within a quasiequilibrium framework. Here, we are capturing the nonlinear response of the $U(1)$ symmetry broken equilibrium ground state. An intense pump pulse is known to weaken this ground state, i.e., it decreases the gap $\Delta$. Strictly speaking the experimental nonlinear response is then measured with respect to the weakened ground state. Our theoretical description does not capture this induced change. It fully loses its validity when the pump induces a phase transition from the superconducting to the normal state [35]. It is nevertheless interesting that present day pump probe experiments are performed in a regime where the induced change of the ground state only quantitatively affects the nonlinear response. Hence, the quasi-equilibrium effective action approach has been established as a description not only of THG but also of pump probe experiments in the literature [35].

\section{Pump-probe spectroscopy}

Higher orders of the optical conductivity include contributions of collective modes that smooth out the absorption edge and add spectral weight inside the conductivity gap. Here, we calculate the nonlinear contribution,

$$
\sigma\left(\omega, \delta t_{p p}\right)=\frac{\left.j(\omega)\right|_{1}+\left.j(\omega)\right|_{3}}{i \omega A(\omega)}
$$

in a pump-probe setting of the time-dependent density-matrix formalism. To this end, we pump the system with an intense pulse of fluence $A_{0}=0.5 \times 10^{-8} \mathrm{Js} / \mathrm{C} / \mathrm{m}$ and, after a delay $\delta t_{p p}$, apply a weak probe pulse. Following experimental schemes [36], we perform two simulations. First, we simulate both a pump and a probe pulse to compute $j_{p p}$. In a second simulation we apply the pump only, obtaining $j_{p}$. We then compute the optical conductivity from the difference in currents $j=j_{p p}-j_{p}$. This ensures that resilient contributions of the pump do not affect the optical conductivity.

Figures 8(a) and 8(b) show the real and imaginary part of the optical conductivity $\sigma\left(\omega, \delta t_{p p}\right)$ as a function of frequency and pump-probe delay. It can be seen that the third-order contribution $\left.j\right|_{3}$ adds spectral weight to the conductivity below absorption gap. The conductivity shows clear oscillations in $\delta t_{p p}$, as emphasized in Figs. 8(c) and 8(e) where only the nonlinear contribution is plotted for the contributions from Higgs and density fluctuations, respectively. A Fourier transform of these oscillations, shown in Figs. 8(d) and 8(e), reveals that the oscillation frequency matches the resonance frequency of the Higgs mode $2 \Delta$. Additionally, the Higgs signal exceeds the density fluctuations by one order of magnitude.

Our results show that signatures of the Higgs mode are measurable in the pump-probe response of the optical conductivity. Yet, to excite the Higgs mode, impurities are crucial. We find that the calculated time-resolved optical response of a single-band superconductor in the dirty-limit is in good agreement with the experimentally measured response [11].
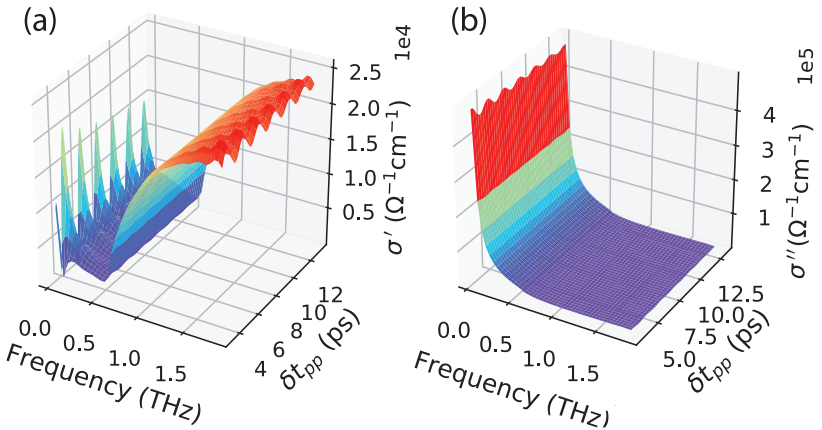

(c)

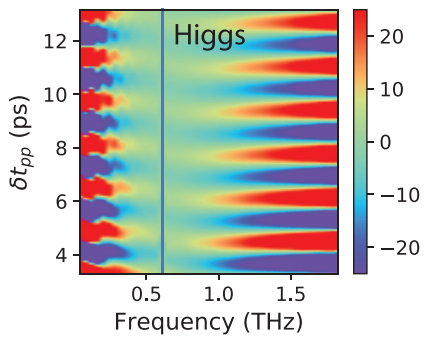

(d)

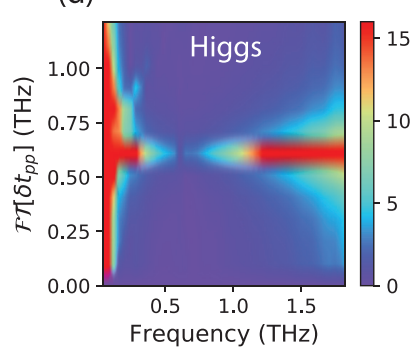

(e)

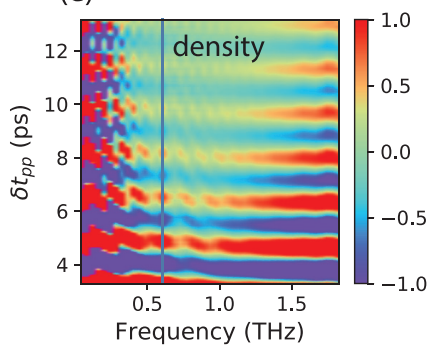

(f)

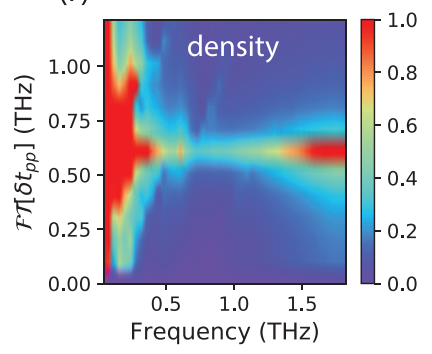

FIG. 8. [(a),(b)] Real and imaginary part of conductivity spectra for sweeped pump-probe delay $\delta t_{\mathrm{pp}}$ including the nonlinear contribution in $A$. (c) Real part of isolated nonlinear Higgs contribution and (d) Fourier transform showing that frequency of conductivity oscillation is peaked at $2 \Delta$. (e) Real part of the nonlinear contribution from density fluctuations and (f) Fourier transform. This Higgs contribution exceeds the charge density contribution by one magnitude.

\section{MULTIBAND SUPERCONDUCTIVITY}

Motivated by the good agreement of the theory with experimental data for a single-band superconductor, we now turn to the case of a two-band superconductor. For concreteness, we focus on the superconducting state of $\mathrm{MgB}_{2}$. We model the $\pi$ and $\sigma$ bands believed to be responsible for superconductivity by choosing material parameters $\Delta_{\pi}=3 \mathrm{meV}, \Delta_{\sigma}=7 \mathrm{meV}$, $\epsilon_{F, \pi}=2.9 \mathrm{eV}, \epsilon_{F, \sigma}=0.7 \mathrm{eV}, m_{\pi}=0.85 m_{e}, m_{\sigma}=1.38 m_{e}$, $\omega_{D}=50 \mathrm{meV}, s_{\pi}=1$, and $s_{\sigma}=-1$ [37].

Convincing evidence for the two-band character of $\mathrm{MgB}_{2}$ has been found in tunneling measurements [38,39] and ARPES [40]. However, optical linear response probes have only revealed signatures of a superconducting gap in the $\pi$ band [17,41]. A recent paper [17] on third-harmonic generation suggests strong evidence of a collective Higgs resonance in the $\pi$ band, but no collective response in the $\sigma$ band was observed. 

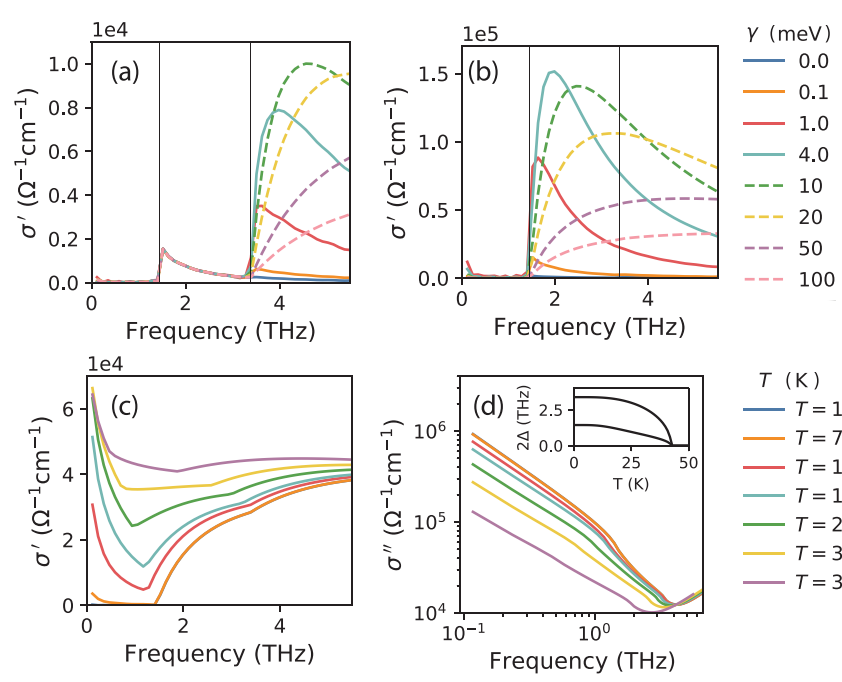

$T(\mathrm{~K})$

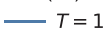

$T=7$

$-T=13$

$T=19$

$-T=25$

$T=31$

$T=37$

FIG. 9. [(a),(b)] Real part $\sigma^{\prime}$ of linear response optical conductivity of a two-band superconductor for various impurity scattering rates at $T=4 \mathrm{~K}$. In panel (a) the impurity of concentration of the first band is $\gamma_{\pi}=0.01 \mathrm{meV}$ and the second-band impurity scattering rates are given by the legend. In panel (b) the legend specifies $\gamma_{\pi}$ and $\gamma_{\sigma}=0.1 \mathrm{meV}$. [(c),(d)] $\sigma^{\prime}$ and $\sigma^{\prime \prime}$ for various temperatures at $\gamma_{1}=100 \mathrm{meV}$ and $\gamma_{2}=50 \mathrm{meV}$. The imaginary part follows a $1 / \omega$ power law at small frequencies. (inset) BCS temperature dependence of the two gaps.

\section{A. Optical conductivity}

The linear response optical conductivity of multiband superconductors is additively composed of contributions from the two bands, $\sigma(\omega)=\sigma_{\pi}+\sigma_{\sigma}$, where the band-specific conductivities are determined by a straightforward generalization of Eq. (19). Figures 9(a) and 9(b) show optical conductivities for various different combinations of band impurity concentrations.

Experimental measurements of the optical conductivity of $\mathrm{MgB}_{2}$ below $T_{C}$ show a clear absorption gap below $2 \Delta_{\pi}$ and a dome shaped onset above $2 \Delta_{\pi}$. A second onset at $\omega=2 \Delta_{\sigma}$ has so far not been observed. Our simulations reproduce these findings in two different parameter regimes: in the dirty-clean limit, where only the first gap contributes to $\sigma(\omega)$, and in the dirty-dirty limit shown in Figs. 9(c) and 9(d). Latter case only shows a weak onset of the $\sigma$ gap, which may be unnoticeable with experimental uncertainties. The reason of the subdominant contribution of the second gap lies in the small Fermi surface of the $\sigma$ band. Explicitly, this can be seen from the prefactor $v_{F_{i}} N_{i}$ in Eq. (19). For our choice of parameters, which include a high estimate of $\epsilon_{F_{\sigma}}$, this yields a suppression of the $\sigma$-gap conductivity by a factor $v_{F_{\pi}} N_{\pi} / v_{F_{\sigma}} N_{\sigma}=6.6$. For a more conservative estimate of $\epsilon_{F_{\sigma}}$, the suppression should be even more pronounced.

\section{B. Collective modes}

Pulse induced changes of the two order parameters $\Delta_{i}$ with $i=\pi, \sigma$ in the two-band case are given by

$$
\begin{aligned}
\delta \Delta_{i}(\omega)= & \frac{1}{2} \sum_{j \mathbf{k k ^ { \prime }}} H_{i j}^{-1}(\omega)\left|J_{j \mathbf{k} \mathbf{k}^{\prime}}\right|^{2} \int d \omega^{\prime} \chi_{j}^{\sigma_{0} \sigma_{0} \sigma_{1}}\left(\omega, \omega^{\prime}, \mathbf{k}, \mathbf{k}^{\prime}\right) \\
& \times A\left(\omega^{\prime}\right) A\left(-\omega-\omega^{\prime}\right),
\end{aligned}
$$

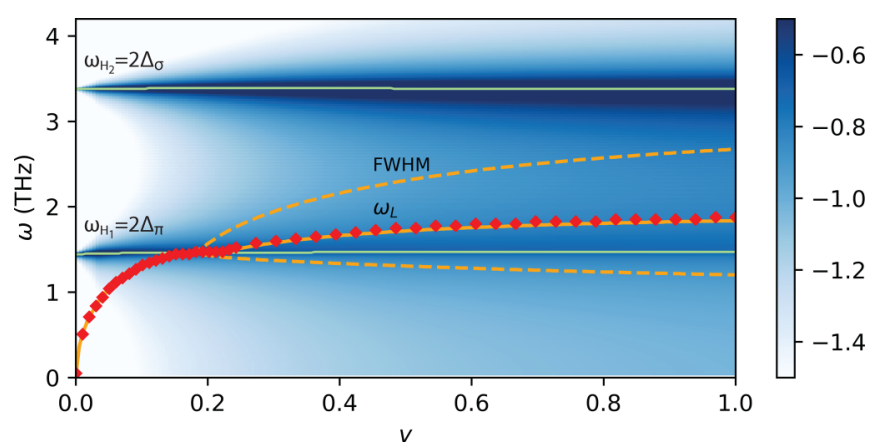

FIG. 10. Logarithmic plot of resonance spectrum of Higgs and Leggett modes as a function of interband coupling parameter $v$. False-color plot was computed within the effective action formalism. Solid-green line shows the frequency of the Higgs resonances. The solid- and dashed-orange lines mark the maximum and width of the Leggett mode. Red diamonds mark the Leggett oscillation frequencies extracted from a pumped time-dependent density-matrix simulation. The two approaches show excellent agreement.

where

$$
H=\left(\begin{array}{cc}
\chi_{1}^{\sigma_{1} \sigma_{1}}+2 U_{22} / \operatorname{det} U & -2 U_{12} / \operatorname{det} U \\
-2 U_{21} / \operatorname{det} U & \chi_{2}^{\sigma_{1} \sigma_{1}}+2 U_{11} / \operatorname{det} U
\end{array}\right)
$$

and where susceptibilities $\chi_{i}^{\sigma_{0} \sigma_{0} \sigma_{1}}, \chi_{i}^{\sigma_{1} \sigma_{1}}$ are listed in Appendix A. The gaps exhibit two resonances, which are determined by the Higgs propagator. In Fig. 10 we show a logarithmic false-color plot of the quantity $|\operatorname{det} H|^{-1}$, responsible for any divergence, as a function of frequency $\omega$ and interband coupling strength $v$. As expected the two resonance energies are at $2 \Delta_{\pi}$ and $2 \Delta_{\sigma}$, illustrated by solid green horizontal lines. Resonances are sharp at small $v$ but decrease and broaden in the strong interband coupling regime.

Energy conservation in Eq. (23) is established by the factor $A\left(\omega^{\prime}\right) A\left(-\omega-\omega^{\prime}\right)$. Oscillation of the gaps is therefore only possible for a finite overlap of $A^{2}(\omega)$ with the resonance frequencies. The matrix structure of $H_{i j}$ further implies that both gaps will oscillate with all excited modes at finite $v$. Dynamics of the phase modes $\theta_{i}$ in the frequency domain are determined by

$$
\delta \theta_{i}(\omega)=\frac{1}{2} \sum_{j} \frac{s_{j} e^{2}}{2 m_{j} \omega^{2}} L_{i j}^{-1}(\omega) \chi_{j}^{\sigma_{3} \sigma_{3}}(\omega) A^{2}(\omega) .
$$

Due to the Anderson-Higgs mechanism only the dynamics of the phase difference $\delta \varphi=\delta \theta_{\pi}-\delta \theta_{\sigma}$ is physical. Inserting Eq. (25) yields the expression

$$
\begin{aligned}
\delta \varphi(\omega)= & \frac{1}{4} A^{2}(\omega)\left(\frac{s_{\pi}}{m_{\pi}}-\frac{s_{\sigma}}{m_{\sigma}}\right) \\
& \times\left[\omega^{2}+\frac{8 \Delta_{\pi} \Delta_{\sigma} v}{U_{\sigma \sigma}-v^{2} U_{\pi \pi}} \frac{\chi_{\pi}^{\sigma_{3} \sigma_{3}}+\chi_{\sigma}^{\sigma_{3} \sigma_{3}}}{\chi_{\pi}^{\sigma_{3} \sigma_{3}} \chi_{\sigma}^{\sigma_{3} \sigma_{3}}}\right]^{-1} .
\end{aligned}
$$

Solid- and dashed-orange lines in Fig. 10 trace the maximum and full width at half $\max (\mathrm{FWHM})$ of $\delta \varphi(\omega) / A^{2}(\omega)$. Red diamonds are the dominant oscillation frequency of the phase

$$
\delta \varphi(t) \approx \frac{\delta \Delta_{\pi}^{\prime \prime}}{\Delta_{\pi}}-\frac{\delta \Delta_{\sigma}^{\prime \prime}}{\Delta_{\sigma}}
$$


(a)

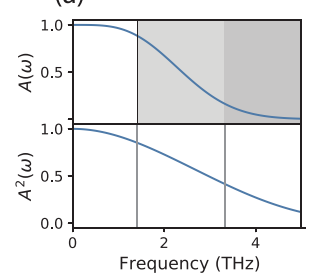

(h)

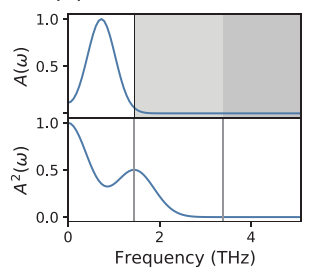

(o)

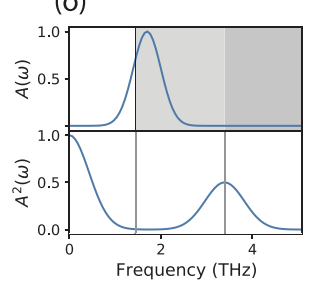

(b)

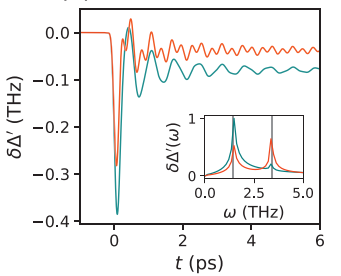

(i)
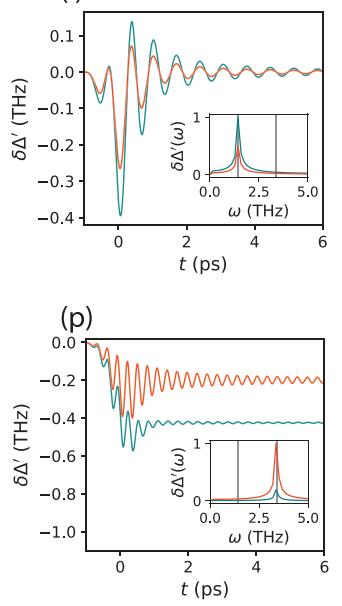

(c)

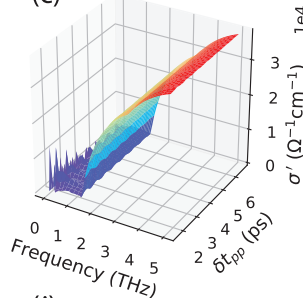

(j)

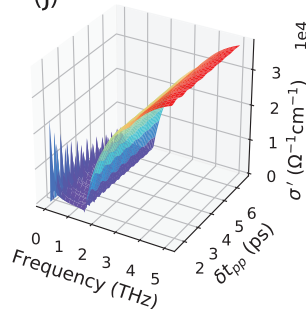

(q)

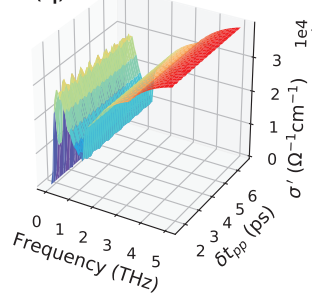

(d)

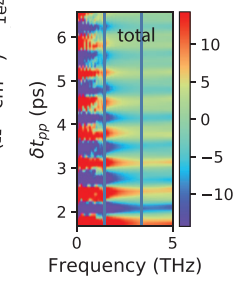

(k)

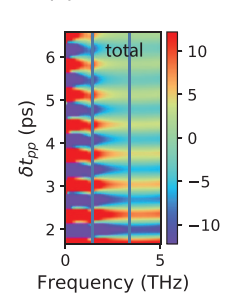

(r)

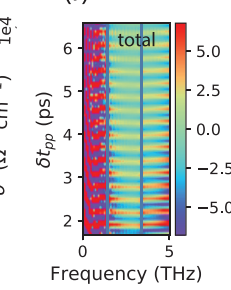

(e)

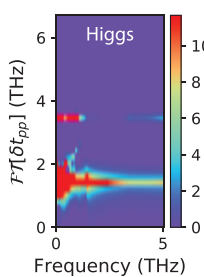

(I)

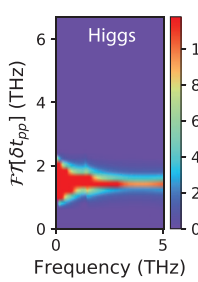

(s)

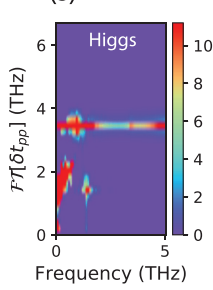

(f)

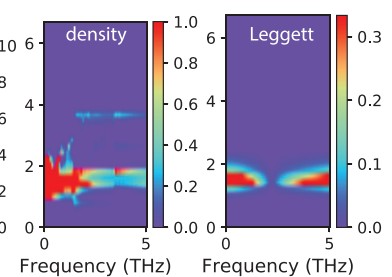

(m)

(n)

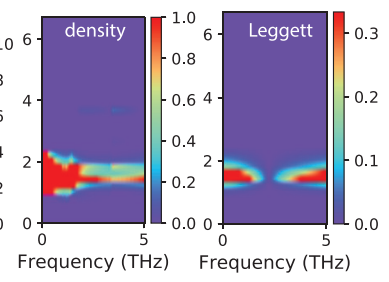

(t)

(u)

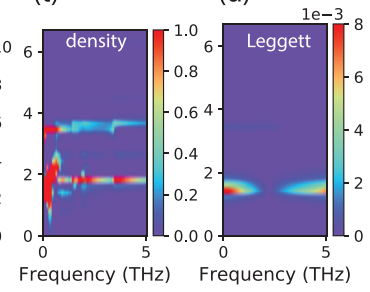

FIG. 11. Time-resolved optical conductivity [(c),(j),(q)] for three optical pulses that resonantly excite (a) both Higgs modes, (h) the lower $\pi$-band Higgs resonance, and (o) the $\sigma$-Higgs mode for an interband coupling strength $v=0.2$ in the dirty-dirty limit with $\gamma_{\pi}=100$ meV and $\gamma_{\sigma}=50 \mathrm{meV}$. [(b),(i),(p)] show the gap oscillations $\delta \Delta^{\prime}(t)$ as a response to the pump pulse only. [(d),(k),(r)] show the background subtracted nonlinear optical conductivity. Their Fourier transforms, separated into Higgs, density fluctuation, and Leggett contributions are shown in the last three columns. The color scales are comparable within each row. They show that the Higgs mode is the dominant contribution for all three pulses whereas the Leggett mode is negligible. The Leggett frequency is identical to $2 \Delta_{\pi}$ for the considered interband coupling $v=0.2$.

evaluated by computing $\delta \Delta_{i}^{\prime \prime}$ in a time-dependent densitymatrix formulation for a broadband optical pulse. The two methods show excellent agreement. At small coupling the phase exhibits completely undamped oscillations due to the absence of decay channels. The Leggett frequency $\omega_{L}$ increases for stronger coupling. Once its energy reaches the quasiparticle threshold it is increasingly damped and the resonance broadens.

The present results reproduce the findings of Refs. [31,42], which were obtained in the clean limit. This should come at no surprise since impurities do not change the frequency of the collective resonance within the MB approach and additionally the Leggett mode only couples diamagnetically to electromagnetic fields.

\section{Pump-probe simulations}

We proceed to model the pump-probe response of a twoband superconductor. Analogous to the single-band case we consider non-linear contributions to the optical conductivity and pump the system with an intense pulse. After some time delay $\delta t_{p p}$, the optical conductivity is probed in the linear response regime by a weak probe pulse.

In Fig. 11 we adopt the dirty-dirty limit with $\gamma_{\pi}=$ $100 \mathrm{meV}$ and $\gamma_{\sigma}=50 \mathrm{meV}$ as a potential description of $\mathrm{MgB}_{2}$ with $v=0.2$ and select various pump pulses shown in the leftmost panels. Gray and dark gray areas illustrate the onset of the quasiparticle continuum of the two bands. Lower panels show $A^{2}(\omega)$ where Higgs resonance frequencies are marked by gray vertical lines. The second column shows the gap dynamics $\delta \Delta_{i}(t)$ following the pump pulse. The third column shows the real part of the time-resolved non-linear optical conductivity $\sigma^{\prime}\left(\omega, \delta t_{p p}\right)$. Panels (d), (k), and (r) show the isolated nonlinear contribution of the real optical conductivity.

The first pump has a broad frequency spectrum such that it overlaps with both Higgs resonances. Following the excitation, both gaps oscillate with both frequencies. The overlap of $A(\omega)$ with the quasiparticle continuum induces a small drop of $\delta \Delta^{\prime}$. The optical conductivity shows oscillations in the pump-probe delay $\delta t_{p p}$ with mostly $2 \Delta_{\pi}$ and a small $2 \Delta_{\sigma}$ component. We attribute the subdominance of the $\delta \Delta_{\sigma}$ contribution to the small $\sigma$-band Fermi surface.

For a narrow-band pulse centered at $\omega=\Delta_{\pi}$ (second row), we observe $2 \Delta_{\pi}$ oscillations only. Here, the pulse $A(\omega)$ does not overlap with the quasiparticle continuum. As a result, the gap oscillates around its equilibrium value $\Delta_{\infty}=\Delta$.

When the narrow-band pulse is centered around the second Higgs resonance at $\omega=2 \Delta_{\sigma}$ (third row), the gap performs $2 \Delta_{\sigma}$ oscillations only. However, the nonlinear current response is weak and numerically hard to resolve.

The last three columns of Fig. 11 show the Fourier transforms of panels (d), (k), and (r), which are further separated into Higgs, charge density, and Leggett contributions. In all cases the current is dominated by the Higgs signal, which 

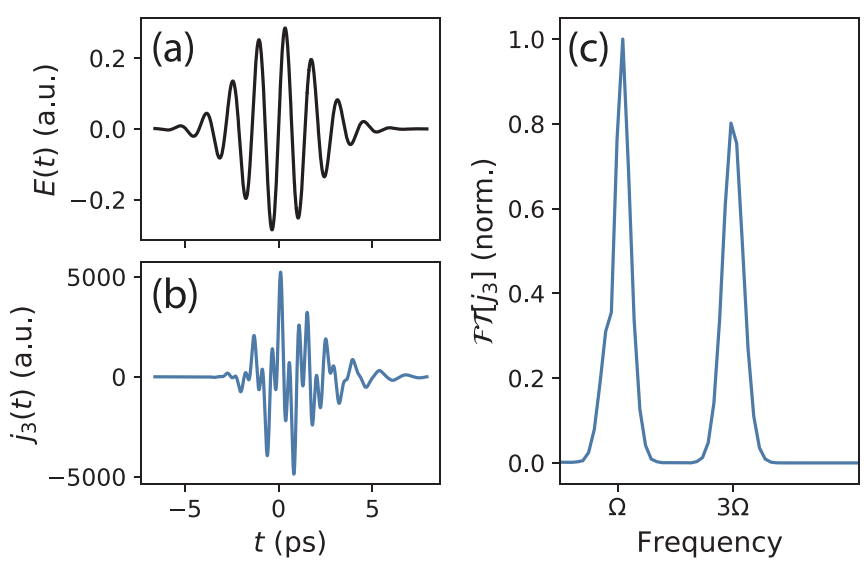

FIG. 12. (a) Realistic multicycle pulse of main frequency $\Omega$ fed into time-dependent density matrix simulation. (b) Simulated thirdorder current $j_{3}(t)$. (c) Next to the original $\Omega$ component, the Fourier transform $\left|j_{3}(\omega)\right|$ reveals an additional $3 \Omega$ component.

exceeds the density fluctuations by roughly one order of magnitude. The Leggett frequency for $v=0.2$ matches the energy of the lower Higgs modes $2 \Delta_{\pi}$. It is, however, always small compared to the Higgs and density fluctuations. In panel Fig. 11(u) the Leggett contribution nearly vanishes, since there is little overlap of the squared pulse $A^{2}(\omega)$ with its resonance frequency.

\section{Third-harmonic generation}

Finally, we simulate the nonlinear response of a multiband superconductor in a THG setup within the time-dependent density-matrix framework. We model a realistic multicycle pulse of frequency $\Omega$, exemplary shown in Fig. 12, and compute the third-order current $\left.j(t)\right|_{3}$. The Fourier transform of $\left.j(t)\right|_{3}$ reveals a $3 \Omega$ third-harmonic (TH) component next to the original first-harmonic $(\mathrm{FH})$ peak.

We adopt the dirty-dirty band description of $\mathrm{MgB}_{2}$ with $\gamma_{\pi}=100 \mathrm{meV}, \gamma_{\sigma}=50 \mathrm{meV}$ and choose two different interband coupling strengths, $v=0.05$ and $v=0.4$. Then, we sweep temperature to investigate the resonant behavior of the TH component. We consider three pulses of frequencies $\Omega=0.5,0.6,0.7 \mathrm{THz}$ and expect the TH component to be resonantly enhanced when $2 \Omega=2 \Delta_{i}$.

Figures 13(a) and 13(b) show the temperature dependence of the BCS gap. Horizontal lines mark pulse frequencies $\Omega$ used in independent simulations. Resonance conditions are satisfied at intersections with a gap. In the second row, Figs. 13(c) and 13(d), the amplitude of the TH peak is found as a function of temperature. In the weak coupling case, $v=$ 0.05 , the THG signal for the lower two frequencies exhibits a pronounced peak at the resonance condition for the lower gap. The THG signal peak of the largest frequency is less pronounced, as this frequency is almost equal to the lower gap for a range of temperatures. We also observe much smaller peaks at temperatures where pulses are in resonance with the larger $\sigma$-gap.

In the strong coupling case, $v=0.4$, we no longer observe a peak-like resonance for the lower $\pi$-band gap. This can be understood as a result of broadening of the Higgs resonance at
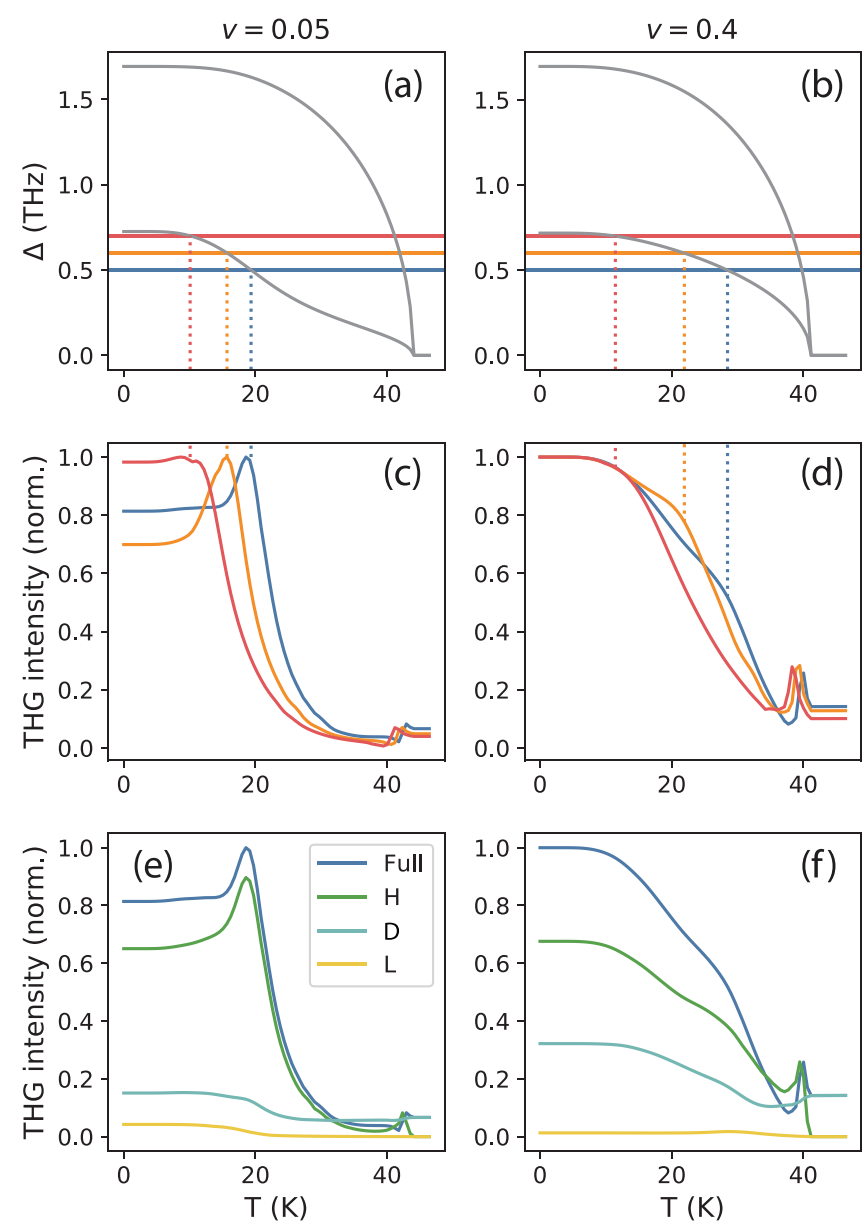

FIG. 13. [(a),(b)] Temperature dependence of the BCS gaps at $v=0.05$ and $v=0.4$. Horizontal lines mark the three pulse frequencies $\Omega=0.5,0.6,0.7 \mathrm{THz}$. [(c),(d)] THG current as a function of temperature for three pulse frequencies $\Omega_{j}$. We take the THG current as $j_{3}(\omega=3 \Omega)$, i.e., the amplitude of the second peak in Fig. 12(c) and sweep temperature. [(e),(f)] Decomposition of the THG signal for pulse of $\Omega=0.5 \mathrm{THz}$ in Higgs $(\mathrm{H})$, density fluctuation (D), and Leggett (L) contributions. The main contribution stems from the collective Higgs mode in both the weak coupling (left) and strong coupling case (right).

large $v$, shown in Fig. 14. Here, we plot the nonlinear Higgs current as a function of temperature $T$ and frequency $\Omega$ computed within the effective action formalism for a sinusoidal excitation. Vertical cuts correspond to the three simulations of Fig. 13. It can be seen that the resonance of the Higgs modes significantly broadens in the strong coupling case $v=0.4$. Thus, the THG signal is already large when driven slightly below the $2 \Delta_{\pi}$ resonance at $T=0$ and no sharp peak occurs when the temperature is increased. This result is further discussed in Appendix D.

The $\sigma$ gap still induces a sharp resonance peak, albeit small in comparison to the low-temperature signal.

Panels (e) and (f) of Fig. 13 decompose the THG signal for the $\Omega=0.5 \mathrm{THz}$ pulse into contributions from the Higgs mode, density fluctuations, and Leggett mode. The Leggett mode contribution is found numerically by considering only the diamagnetic component of the current $\left.j_{D}\right|_{3}$. The 

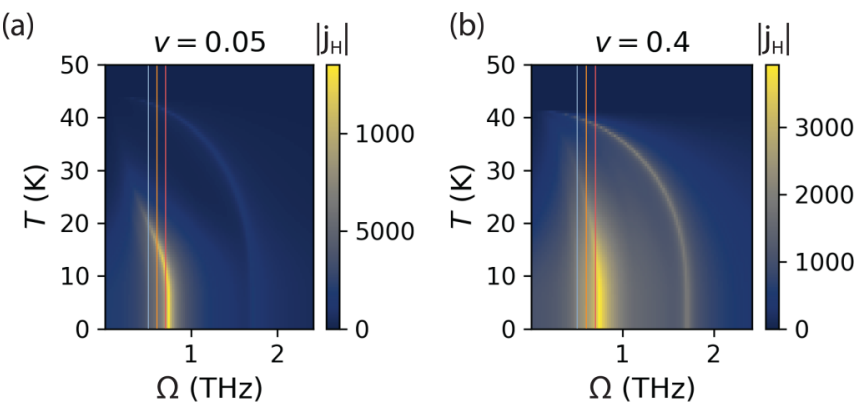

FIG. 14. Nonlinear Higgs current as a function of temperature $T$ and frequency $\Omega$ of a sinusoidal pulse computed within the effective action formalism (a) for $v=0.05$ and (b) for $v=0.4$. Vertical lines represent cuts according to the simulations in Fig. 13.

density-fluctuation contribution is found by forcing $\delta \Delta_{i}=0$ when solving the equations of motion, removing the selfconsistency condition that induces collective modes. In both the weak coupling and large- $v$ case the THG response is dominated by the Higgs mode. The relative contribution of density fluctuations increases in the strong interband coupling regime. The Leggett contribution is vanishingly small.

The present results are interesting when compared to the experimental findings of Ref. [17]. Our results affirm the claim that the THG response is mainly attributed to the Higgs resonance of the $\pi$ band. The small contribution of the the $\sigma$-band Higgs mode and the Leggett mode in our simulation is consistent with the experiment where no signatures of the Leggett or second Higgs mode were observed. We have further computed the THG response in the dirty-clean limit where we found nearly identical results, apart from the absence of the small $\sigma$ resonance peak at temperatures close to $T_{C}$.

The failure of our theory to produce resonance peaks of the $\pi$-Higgs mode at large $v$ suggests that the MB approximation might not correctly describe the THG response in the strong coupling limit as assumed for $\mathrm{MgB}_{2}$ [31,43]. A recent study has found that incorporating impurities beyond Mattis-Bardeen as random onsite-energies in a lattice model shows a stronger contribution of density fluctuations [23]. This, however, is beyond the scope of this paper and will be explored in future investigations.

\section{CONCLUSION}

We have calculated the time-resolved optical response of dirty multiband superconductors. We have incorporated impurity scattering within the Mattis-Bardeen approximation that effectively broadens the photon momentum distribution of the optical pulse. This approach is known to accurately describe superconductors $[11,44]$, yet deviations in the strong disorder regime are possible $[25,45,46]$. The response was calculated within two different frameworks. First, the time evolution of the system after an excitation with a light pulse was calculated explicitly using a time-dependent density-matrix formalism. Here, the Mattis-Bardeen ansatz enters through a replacement of the matrix element of the current operator with a
Lorentzian-shaped momentum transfer distribution [20]. In the second approach, we calculated the relevant susceptibilities in a diagrammatic formalism derived from an effective action approach. Here, impurities enter through a paramagnetic electromagnetic coupling vertex that carries external momentum. As a consequence, additional diagrams arise that usually vanish in the clean limit. While both approaches yield equivalent results, the diagrammatic approach allows to understand the relevant processes in more detail and is numerically more efficient in certain cases.

In accordance with previous literature [19,20,23], we find that the collective Higgs response is drastically enhanced even for small impurity concentrations. The Leggett mode is unaffected by impurity scattering and hence becomes subdominant. This may change slightly when realistic, nonparabolic band structures and weak violations of particle-hole symmetry are taken into account. An interesting further question is the inclusion of Coulomb interactions within the MB approach.

As a first result, we calculated the dynamics of superconducting order parameter of a single-band superconductor in the dirty limit excited by a short $\mathrm{THz}$ quench pulse. Using a second probe-pulse after a variable time delay, we further computed the time-resolved optical conductivity. Both quantities show oscillations with the Higgs frequency $\omega_{H}=2 \Delta$. The optical response is in good agreement to measurements on $\mathrm{NbN}$ [11].

Extending the model to two bands, we studied pump-probe optical conductivities of the model as an effective low-energy description of $\mathrm{MgB}_{2}$ for various impurity limits of the $\pi$ and $\sigma$ band. We found that experimental results are well reproduced either when both bands are dirty, or when only the lower $\pi$ band is dirty. Here, the collective contribution to the nonlinear optical response is always dominated by the amplitude mode of $\Delta_{\pi}$.

Finally, we presented the third-harmonic generation (THG) response of the two-band model. Interestingly, results obtained in the weak interband coupling regime seem to match available experimental data, showing a pronounced THG resonance mostly due to the $\pi$-band Higgs mode [17]. However, our theory shows deviations in the strong interband coupling case, believed to be representative of $\mathrm{MgB}_{2}$, where no obvious $\pi$-Higgs-resonance is present. We additionally calculated the full temperature and frequency THG signal, which allows a better comparison to current experimental setups.

In summary, we have presented simulations of timeresolved optical conductivities and modelled THG resonance experiments within the Mattis-Bardeen approximation using both a diagrammatic approach and a time-dependent densitymatrix formalism for single-band and two-band superconductors. As studies of collective excitations in superconductors with $\mathrm{THz}$ spectroscopy become more and more common, it is important to understand the correct excitation scheme in the presence of impurities. The Mattis-Bardeen approximation, as shown in this paper for either a density-matrix formalism or a diagrammatic calculation, allows to describe the effects of impurities in a simple way. This will help further studies in achieving more realistic models of experimental results. 


\section{ACKNOWLEDGMENTS}

We are indebted to M. Daghofer, M. Puviani, A. Schnyder, and R. Shimano for illuminating discussions. We thank the Max Planck-UBC-UTokyo Center for Quantum Materials for fruitful collaborations and financial support. R.H. acknowl- edges the Joint-PhD program of the University of British Columbia and the University of Stuttgart. P.F. thanks the UBC Science Co-op program for making an internship possible, and acknowledges computational resources provided by Advanced Research Computing at the University of British Columbia.

\section{APPENDIX A: DERIVATION OF THE EFFECTIVE ACTION}

The problem is stated with the partition function

$$
\mathcal{Z}=\int \mathcal{D}\left(c^{\dagger} c\right) e^{-S} \text { with } \quad S=\int_{0}^{\beta} d \tau\left(\sum_{i \mathbf{k} \sigma} c_{i \mathbf{k} \sigma}^{\dagger} \partial_{\tau} c_{i \mathbf{k} \sigma}+\mathcal{H}\right) .
$$

We decouple the interacting term in the pairing channel via the Hubbard Stratonovich transformation

$$
\begin{aligned}
& \exp \left(\int d \tau \sum_{i j}\left(\sum_{\mathbf{k}} c_{i \mathbf{k} \uparrow}^{\dagger} c_{i-\mathbf{k} \downarrow}^{\dagger}\right) U_{i j}\left(\sum_{\mathbf{k}^{\prime}} c_{j-\mathbf{k}^{\prime} \downarrow} c_{j \mathbf{k}^{\prime} \uparrow}\right)\right) \\
& =\int \mathcal{D}\left(\bar{\Delta}_{i} \Delta_{i}\right) \exp \left(-\int d \tau\left[\sum_{i j} \bar{\Delta}_{i} U_{i j}^{-1} \Delta_{j}-\sum_{i \mathbf{k}}\left(\bar{\Delta}_{i} c_{i \mathbf{k} \uparrow} c_{i-\mathbf{k} \downarrow}+\Delta_{i} c_{i-\mathbf{k} \downarrow}^{\dagger} c_{i \mathbf{k} \uparrow}^{\dagger}\right)\right]\right) .
\end{aligned}
$$

The bosonic fields $\Delta_{i}(\tau)$ are complex, i.e., they permits amplitude and phase fluctuations.

Note that $\Delta_{i}(\tau)$ does not depend on momentum. This is because the usual BCS Hamiltonian, Eq. (1), is an approximation of the more general interaction term $\sum_{i j \mathbf{k} \mathbf{k}^{\prime} \mathbf{q}} U_{i j} c_{i \mathbf{k}+\mathbf{q} \uparrow}^{\dagger} c_{i-\mathbf{k} \downarrow}^{\dagger} c_{j-\mathbf{k}^{\prime} \downarrow} c_{j \mathbf{k}^{\prime}+\mathbf{q} \uparrow}$, where the Cooper pair center of mass momentum is incorporated through the summation over the small variable q. It is interesting to note that a supercurrent can be modelled even in the absence of such a center of mass momentum. In that case, the supercurrent is determined by the static component of the electromagnetic vector potential $[47,48]$.

We decompose $\Delta_{i}$ into real fields and additional express fluctuation with respect to the meanfield saddlepoint, $\Delta_{i}(\tau) \rightarrow$ $\left(\Delta_{i}^{e q}+\Delta_{i}(\tau)\right) e^{i \theta_{i}(\tau)}, \bar{\Delta}_{i}(\tau) \rightarrow\left(\Delta_{i}^{e q}+\Delta_{i}(\tau)\right) e^{-i \theta_{i}(\tau)}$. The action is

$$
\begin{aligned}
S= & \sum_{i j} U_{i j}^{-1} \int d \tau\left(\Delta_{i}^{e q} \Delta_{j}^{e q}+\Delta_{i}(\tau) \Delta_{j}(\tau)\right) e^{-i\left(\theta_{i}(\tau)-\theta_{j}(\tau)\right)} \\
& +\sum_{i \mathbf{k} \sigma} \int_{0}^{\beta} d \tau\left(c_{i \mathbf{k} \sigma}^{\dagger}\left[\partial_{\tau}+\xi_{i \mathbf{k}}\right] c_{i \mathbf{k} \sigma}-\left(\Delta_{i}^{e q}+\Delta_{i}(\tau)\right) e^{-i \theta_{i}(\tau)} c_{i \mathbf{k} \uparrow} c_{i-\mathbf{k} \downarrow}-\left(\Delta_{i}^{e q}+\Delta_{i}(\tau)\right) e^{i \theta_{i}(\tau)} c_{i-\mathbf{k} \downarrow}^{\dagger} c_{i \mathbf{k} \uparrow}^{\dagger}\right)+\int_{0}^{\beta} d \tau \mathcal{H}_{1} .
\end{aligned}
$$

Here we have encountered the Josephson coupling term

$$
\sum_{i j} U_{i j}^{-1} \Delta_{i}^{e q} \Delta_{j}^{e q} e^{-i\left(\theta_{i}(\tau)-\theta_{j}(\tau)\right)}=U_{11}^{-1}\left(\Delta_{1}^{e q}\right)^{2}+U_{22}^{-1}\left(\Delta_{2}^{e q}\right)^{2}+2 U_{12}^{-1} \Delta_{1}^{e q} \Delta_{2}^{e q} \cos \left(\theta_{1}-\theta_{2}\right)
$$

that induces the Leggett mode. We express the action in the Nambu basis $\Psi_{i \mathbf{k}}^{\dagger}\left(\omega_{n}\right)=\left(c_{i \mathbf{k} \uparrow}^{\dagger}, c_{i-\mathbf{k} \downarrow}\right)$,

$$
S=\sum_{i j} U_{i j}^{-1} \int d \tau\left(\Delta_{i}^{e q} \Delta_{j}^{e q}+\Delta_{i}(\tau) \Delta_{j}(\tau)\right) e^{-i\left(\theta_{i}-\theta_{j}\right)}-\sum_{i \mathbf{k k}^{\prime}} \int d \tau \Psi_{i \mathbf{k}}^{\dagger}(\tau) G_{i}^{-1}\left(\mathbf{k k}^{\prime}, \tau\right) \Psi_{i \mathbf{k}^{\prime}}(\tau),
$$

where

$$
G_{i}^{-1}=\delta_{\mathbf{k k}^{\prime}}\left(\begin{array}{cc}
-\partial_{\tau}-\xi_{i \mathbf{k}} & \left(\Delta_{i}^{e q}+\Delta_{i}(\tau)\right) e^{i \theta(\tau)} \\
\left(\Delta_{i}^{e q}+\Delta_{i}(\tau)\right) e^{-i \theta(\tau)} & -\partial_{\tau}+\xi_{i \mathbf{k}}
\end{array}\right)+J_{i \mathbf{k k}^{\prime}} \cdot \mathbf{e} A(\tau) \sigma_{0}-\frac{s_{i} e^{2}}{2 m_{i}} A^{2}(\tau) \delta_{\mathbf{k k}^{\prime}} \sigma_{3} .
$$

Integrating out the Fermions gives

$$
S=\sum_{i j} U_{i j}^{-1} \int d \tau\left(\Delta_{i}^{e q} \Delta_{j}^{e q}+\Delta_{i}(\tau) \Delta_{j}(\tau)\right) e^{-i\left(\theta_{i}-\theta_{j}\right)}-\sum_{i} \operatorname{Tr} \ln \left[-G_{i}^{-1}\right],
$$

where the trace is performed over time, momenta, and Nambu indices, but not over band-indices $i$. To separate amplitude $\Delta_{i}(\tau)$ and phase $\theta_{i}(\tau)$ fields, we introduce a local unitary transformation $V_{i}=\exp \left(i \theta_{i}(\tau) \sigma_{3} / 2\right)$ [49],

$$
\operatorname{Tr} \ln \left[-G_{i}^{-1}\right]=\operatorname{Tr} \ln \left[-G_{i}^{-1} V_{i} V_{i}^{\dagger}\right]=\operatorname{Tr} \ln \left[-V_{i}^{\dagger} G_{i}^{-1} V_{i}\right]=\operatorname{Tr} \ln \left[-\tilde{G}_{i}^{-1}\right]
$$


We split $\tilde{G}_{i}^{-1}$ into a meanfield part, $G_{0, i}^{-1}$, and all remaining contributions $\Sigma_{i}$. In frequency space, this gives

$$
\begin{gathered}
\tilde{G}_{i}^{-1}=G_{0, i}^{-1}-\Sigma_{i}, \\
G_{0, i}^{-1}\left(\mathbf{k} \omega_{n}, \mathbf{k}^{\prime} \omega_{m}\right)=\left[i \omega_{n}-\xi_{i \mathbf{k}} \sigma_{3}+\Delta_{i}^{e q} \sigma_{1}\right] \delta_{\mathbf{k} \mathbf{k}^{\prime}} \delta_{\omega_{n}, \omega_{m}}, \\
\Sigma_{i}\left(\mathbf{k} \omega_{n}, \mathbf{k}^{\prime} \omega_{m}\right)=-\Delta_{i}\left(\omega_{n}-\omega_{m}\right) \sigma_{1} \delta_{\mathbf{k} \mathbf{k}^{\prime}}-i \frac{i \omega_{n}-i \omega_{m}}{2} \theta_{i}\left(\omega_{n}-\omega_{m}\right) \sigma_{3} \delta_{\mathbf{k} \mathbf{k}^{\prime}}-J_{i \mathbf{k} \mathbf{k}^{\prime}} \cdot \mathbf{e} A\left(\omega_{n}-\omega_{m}\right) \sigma_{0}+\frac{s_{i} e^{2}}{2 m_{i}} A^{2}\left(\omega_{n}-\omega_{m}\right) \delta_{\mathbf{k} \mathbf{k}^{\prime}} \sigma_{3} .
\end{gathered}
$$

Note that phase fluctuations $\theta_{i}$ live in the $\sigma_{3}$ channel, i.e., the charge channel.

Next, we expand $S$ at Gaussian level. To compute currents $\left.j\right|_{1}$ and $\left.j\right|_{3}$ we additionally keep terms up to fourth order in the classical field $A$. In expanding the trace, we use

$$
\operatorname{Tr} \ln \left(-\tilde{G}^{-1}\right)=\operatorname{Tr} \ln \left(-G_{0}^{-1}\left(1-G_{0} \Sigma\right)\right)=\operatorname{Tr} \ln \left(-G_{0}^{-1}\right)-\operatorname{Tr} \sum_{n=1}^{\infty} \frac{1}{n}\left(G_{0} \Sigma\right)^{n} .
$$

The quadratic action is given by the terms

$$
S\left[\Delta_{i}, \theta_{i}, A\right]=S_{M F}+S_{\Delta}+S_{\Delta, A}+S_{\theta}+S_{\theta, A}+S_{\mathrm{QP}, \mathrm{dia}}+S_{\mathrm{QP}, \mathrm{para}},
$$

which are explicitly

$$
\begin{aligned}
& S_{M F}=\sum_{i j} U_{i j}^{-1} \Delta_{i}^{e q} \Delta_{j}^{e q}-\sum_{i} \operatorname{Tr} \ln \left[-G_{0, i}^{-1}\right] \\
& S_{\Delta}=\frac{1}{2} \sum_{i j \Omega_{m}} \Delta_{i}\left(-\Omega_{m}\right)\left(\begin{array}{cc}
\chi_{1}^{\sigma_{1} \sigma_{1}}\left(\Omega_{m}\right)+2 U_{22} / \operatorname{det} U & -2 U_{12} / \operatorname{det} U \\
-2 U_{21} / \operatorname{det} U & \chi_{2}^{\sigma_{1} \sigma_{1}}\left(\Omega_{m}\right)+2 U_{11} / \operatorname{det} U
\end{array}\right)_{i j} \Delta_{j}\left(\Omega_{m}\right) \\
& =\frac{1}{2} \sum_{i j \Omega_{m}} \Delta_{i}\left(-\Omega_{m}\right) H_{i j}^{-1}\left(\Omega_{m}\right) \Delta_{j}\left(\Omega_{m}\right), \\
& S_{\Delta, A}=-\sum_{i \mathbf{k} \mathbf{k}^{\prime}} \sum_{\Omega_{m} \Omega_{l}}\left|J_{i \mathbf{k} \mathbf{k}^{\prime}}\right|^{2} \chi_{i}^{\sigma_{0} \sigma_{0} \sigma_{1}}\left(\Omega_{m}, \Omega_{l}, \mathbf{k}, \mathbf{k}^{\prime}\right) A\left(\Omega_{l}\right) A\left(-\Omega_{m}-\Omega_{l}\right) \Delta\left(\Omega_{m}\right), \\
& S_{\theta}=-\frac{1}{2} \sum_{i j \Omega_{m}} \frac{-i \Omega_{m}}{2} \theta_{i}\left(-\Omega_{m}\right)\left(\begin{array}{cc}
-\chi_{1}^{\sigma_{3} \sigma_{3}}\left(\Omega_{m}\right)+\frac{\lambda}{\Omega_{m}^{2}} & -\frac{\lambda}{\Omega_{m}^{2}} \\
-\frac{\lambda}{\Omega_{m}^{2}} & -\chi_{2}^{\sigma_{3} \sigma_{3}}\left(\Omega_{m}\right)+\frac{\lambda}{\Omega_{m}^{2}}
\end{array}\right)_{i j} \frac{i \Omega_{m}}{2} \theta_{j}\left(\Omega_{m}\right) \\
& =-\frac{1}{2} \sum_{i j \Omega_{m}} \frac{-i \Omega_{m}}{2} \theta_{i}\left(-\Omega_{m}\right)\left[\left(\begin{array}{ll}
-\chi_{1}^{\sigma_{3} \sigma_{3}}\left(\Omega_{m}\right) & -\chi_{2}^{\sigma_{3} \sigma_{3}}\left(\Omega_{m}\right)
\end{array}\right)_{i j}+J_{i j}\right] \frac{i \Omega_{m}}{2} \theta_{j}\left(\Omega_{m}\right) \\
& =\frac{1}{2} \sum_{i j \Omega_{m}} \frac{-i \Omega_{m}}{2} \theta_{i}\left(-\Omega_{m}\right) L_{i j}^{-1}\left(\Omega_{m}\right) \frac{i \Omega_{m}}{2} \theta_{j}\left(\Omega_{m}\right) \text {, } \\
& S_{\theta, A}=\sum_{i \Omega_{m}} i \frac{s_{i} e^{2}}{2 m_{i}} \mathbf{A}^{2}\left(\Omega_{m}\right) \chi_{i}^{\sigma_{3} \sigma_{3}}\left(\Omega_{m}\right) \frac{-i \Omega_{m}}{2} \theta_{i}\left(-\Omega_{m}\right), \\
& S_{\mathrm{QP}, \mathrm{dia}}^{(2)}=-\sum_{i} \frac{s_{i} e^{2}}{2 m_{i}} \chi_{i}^{\sigma_{3}} A^{2}(0), \\
& S_{\mathrm{QP}, \mathrm{dia}}^{(4)}=\frac{1}{2} \sum_{i \Omega_{m}}\left(\frac{s_{i} e^{2}}{2 m_{i}}\right)^{2} \chi_{i}^{\sigma_{3} \sigma_{3}}\left(\Omega_{m}\right) A^{2}\left(\Omega_{m}\right) A^{2}\left(-\Omega_{m}\right), \\
& S_{\mathrm{QP}, \text { para }}^{(2)}=\frac{1}{2} \sum_{i \mathbf{k} \mathbf{k}^{\prime} \Omega_{m}}\left|J_{i \mathbf{k} \mathbf{k}^{\prime}}\right|^{2} \chi_{i}^{\sigma_{0} \sigma_{0}}\left(\mathbf{k}, \mathbf{k}^{\prime}, \Omega_{m}\right) A\left(\Omega_{m}\right) A\left(-\Omega_{m}\right), \\
& S_{\mathrm{QP}, \mathrm{para}}^{(4)}=\frac{1}{4} \sum_{i \mathbf{k} \mathbf{k}^{\prime} \mathbf{k}^{\prime \prime}} \sum_{\Omega_{m} \Omega_{l} \Omega_{p}}\left|J_{i \mathbf{k} \mathbf{k}^{\prime}}\right|^{2}\left|J_{i \mathbf{k} \mathbf{k}^{\prime \prime}}\right|^{2} \xi_{i}\left(\mathbf{k}, \mathbf{k}^{\prime}, \mathbf{k}^{\prime \prime}, \Omega_{m}, \Omega_{l}, \Omega_{p}\right) A\left(\Omega_{m}\right) A\left(\Omega_{l}\right) A\left(\Omega_{p}\right) A\left(-\Omega_{m}-\Omega_{l}-\Omega_{p}\right) .
\end{aligned}
$$

Here, $\lambda=\frac{8 \Delta_{1} \Delta_{2} v}{U_{22}-v^{2} U_{11}}$. The Higgs and Leggett terms, Eqs. (A16)-(A18), are diagrammatically shown in Fig. 1. The densityfluctuation terms, Eqs. (A19)-(A22) are shown in Fig. 2. The susceptibilities, given by the fermionic bubbles, are

$$
\chi_{i}^{\sigma_{k}}=\sum_{\mathbf{k}} \sum_{\omega_{n}} \operatorname{Tr}\left[G_{0, i}\left(\omega_{n}, \mathbf{k}\right) \sigma_{k}\right]
$$


(a)

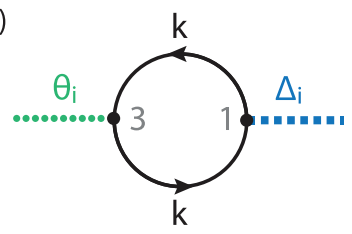

(b)

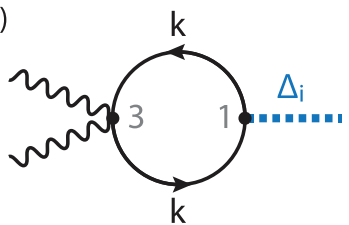

(c)

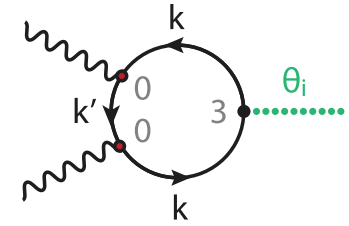

(d)

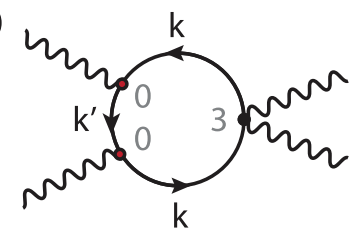

FIG. 15. Additional diagrams following from Eq. (A7) that vanish in the presence of particle-hole symmetry and a parabolic dispersion.

$$
\begin{gathered}
\chi_{i}^{\sigma_{k} \sigma_{l}}\left(\Omega_{m}, \mathbf{k}, \mathbf{k}^{\prime}\right)=\sum_{\omega_{n}} \operatorname{Tr}\left[G_{0, i}\left(\omega_{n}, \mathbf{k}\right) \sigma_{k} G_{0, i}\left(\omega_{n}+\Omega_{m}, \mathbf{k}^{\prime}\right) \sigma_{l}\right], \\
\chi_{i}^{\sigma_{k} \sigma_{l}}\left(\Omega_{m}\right)=\sum_{\mathbf{k}} \chi_{i}^{\sigma_{k} \sigma_{l}}\left(\Omega_{m}, \mathbf{k}, \mathbf{k}\right), \\
\chi_{i}^{\sigma_{0} \sigma_{0} \sigma_{1}}\left(\Omega_{m}, \Omega_{l}, \mathbf{k}, \mathbf{k}^{\prime}\right)=\sum_{\omega_{n}} \operatorname{Tr}\left[G_{0, i}\left(\omega_{n}+\Omega_{m}, \mathbf{k}\right) G_{0, i}\left(\omega_{n}+\Omega_{m}+\Omega_{l}, \mathbf{k}^{\prime}\right) G_{0, i}\left(\omega_{n}, \mathbf{k}\right) \sigma_{1}\right] \\
\xi_{i}\left(\mathbf{k}, \mathbf{k}^{\prime}, \mathbf{k}^{\prime \prime}, \Omega_{m}, \Omega_{l}, \Omega_{p}\right)=\sum_{\omega_{n}} \operatorname{Tr}\left[G_{0, i}\left(\omega_{n}, \mathbf{k}\right) G_{0, i}\left(\omega_{n}+\Omega_{m}, \mathbf{k}^{\prime}\right) G_{0, i}\left(\omega_{n}+\Omega_{m}+\Omega_{l}, \mathbf{k}\right) G_{0, i}\left(\omega_{n}+\Omega_{m}+\Omega_{l}+\Omega_{p}, \mathbf{k}^{\prime \prime}\right)\right],
\end{gathered}
$$

We evaluate Matsubara sums in above expressions analytically using the MatsubaraSum package developed by [50].

Additional diagrams, that vanish due to particle-hole symmetry, are listed in Fig. 15. We proceed to integrate out all collective fields. This gives

$$
S[A]=S_{M F}+\tilde{S}_{\Delta}+\tilde{S}_{\theta}+S_{\mathrm{QP}, \mathrm{dia}}+S_{\mathrm{QP}, \mathrm{para}}
$$

with

$$
\begin{aligned}
\tilde{S}_{\Delta}= & -\frac{1}{2} \sum_{i j \Omega_{m}}\left(\sum_{\mathbf{k k}^{\prime} \Omega_{l}}\left|J_{i \mathbf{k} \mathbf{k}^{\prime}}\right|^{2} \chi_{i}^{\sigma_{0} \sigma_{0} \sigma_{1}}\left(-\Omega_{m}, \Omega_{l}, \mathbf{k}, \mathbf{k}^{\prime}\right) A\left(\Omega_{l}\right) A\left(\Omega_{m}-\Omega_{l}\right)\right) H_{i j}\left(\Omega_{m}\right) \\
& \times\left(\sum_{\mathbf{k} \mathbf{k}^{\prime} \Omega_{l}}\left|J_{j \mathbf{k} \mathbf{k}^{\prime}}\right|^{2} \chi_{j}^{\sigma_{0} \sigma_{0} \sigma_{1}}\left(\Omega_{m}, \Omega_{l}, \mathbf{k}, \mathbf{k}^{\prime}\right) A\left(\Omega_{l}\right) A\left(-\Omega_{m}-\Omega_{l}\right)\right), \\
\tilde{S}_{\theta} & =\frac{1}{2} \sum_{i j \Omega_{m}} \frac{s_{i} e^{2}}{2 m_{i}} \frac{s_{j} e^{2}}{2 m_{j}} A^{2}\left(-\Omega_{m}\right) A^{2}\left(\Omega_{m}\right) \chi_{i}^{\sigma_{3} \sigma_{3}}\left(-\Omega_{m}\right) L_{i j}\left(\Omega_{m}\right) \chi_{j}^{\sigma_{3} \sigma_{3}}\left(\Omega_{m}\right) .
\end{aligned}
$$

We note that phase and fourth-order diamagnetic density-fluctuation terms combine to give the Leggett contribution

$$
\tilde{S}_{L}=S_{Q P, \mathrm{dia}}^{(4)}+\tilde{S}_{\theta}=-\frac{e^{4}}{2} \sum_{\Omega_{m}} \frac{\lambda}{4}\left(\frac{s_{1}}{m_{1}}-\frac{s_{2}}{m_{2}}\right)^{2}\left[\Omega_{n}^{2}-\lambda \frac{\chi_{1}^{\sigma_{3} \sigma_{3}}+\chi_{2}^{\sigma_{3} \sigma_{3}}}{\chi_{1}^{\sigma_{3} \sigma_{3}} \chi_{2}^{\sigma_{3} \sigma_{3}}}\right]^{-1} A^{2}\left(-\Omega_{m}\right) A^{2}\left(\Omega_{m}\right) .
$$

Thus, the zero-energy Goldstone mode does not contribute to the optical response. Above equations, obtained by Gaussian integration, have the diagrammatic representation of an RPA summation shown in Fig. 3. For the Higgs propagator this can be seen by expanding

$$
H=\left[2 U^{-1}+X\right]^{-1}=\frac{U}{2} \sum_{n=0}^{\infty}\left(-X \frac{U}{2}\right)^{n}
$$

where $X_{i j}=\chi_{i}^{\sigma_{1} \sigma_{1}} \delta_{i j}$ corresponds to fermionic bubbles and $U_{i j} / 2$ corresponds to the to dashed lines. The case of the Leggett mode is analogous. The currents can now, after analytic continuation of all external frequencies, be computed by a functional derivative of the action,

$$
j(t)=\frac{\delta}{\delta A(t)} \ln Z[A]=-\frac{\delta}{\delta A(t)} S[A]
$$

In Fourier space this results in

$$
j(-\omega)=-\frac{\delta S[A]}{\delta A(\omega)}
$$


Latter equality follows in full generality from the chain rule of functional derivation,

$$
\frac{\delta S[A]}{\delta \tilde{A}(\omega)}=\frac{\delta}{\delta \tilde{A}(\omega)} S[\mathcal{F} \mathcal{T}[\tilde{A}]]=\int d t \underbrace{\frac{\delta S[A]}{\delta A(t)}}_{-j(t)} \underbrace{\frac{\delta \mathcal{F} \mathcal{T}^{-1}[\tilde{A}](t)}{\delta \tilde{A}(\omega)}}_{e^{-i \omega t}}=-j(-\omega),
$$

where we have denoted the Fourier transform $\tilde{A}(\omega)=\mathcal{F} \mathcal{T}[A](\omega)$ by a tilde.

The Mattis-Bardeen approximation enters by replacing

$$
\sum_{\mathbf{k} \mathbf{k}^{\prime}}\left|J_{i \mathbf{k} \mathbf{k}^{\prime}}\right|=N_{i}(0)^{2} \int d \epsilon_{\mathbf{k}} d \epsilon_{\mathbf{k}^{\prime}} \int \frac{d \Omega_{\mathbf{k}}}{4 \pi} \frac{d \Omega_{\mathbf{k}^{\prime}}}{4 \pi}\left|J_{i \mathbf{k} \mathbf{k}^{\prime}}\right| \approx N_{i}(0)^{2} \int d \epsilon_{\mathbf{k}} d \epsilon_{\mathbf{k}^{\prime}} \frac{\left(e v_{F_{i}}\right)^{2}}{3 N_{i}(0)} W\left(\epsilon_{i \mathbf{k}}, \epsilon_{i \mathbf{k}^{\prime}}\right)
$$

according to Eq. (5). For the fourth-order paramagnetic density-fluctuation contribution, Eq. (A22), we follow Ref. [20] and further approximate

$$
\sum_{\mathbf{k} \mathbf{k}^{\prime} \mathbf{k}^{\prime \prime}}\left|J_{i \mathbf{k} \mathbf{k}^{\prime}}\right|\left|J_{i \mathbf{k} \mathbf{k}^{\prime \prime}}\right| \approx N_{i}(0)^{3} \int d \epsilon_{\mathbf{k}} d \epsilon_{\mathbf{k}^{\prime}} d \epsilon_{\mathbf{k}^{\prime \prime}}\left(\int \frac{d \Omega_{\mathbf{k}}}{4 \pi} \frac{d \Omega_{\mathbf{k}^{\prime}}}{4 \pi}\left|J_{i \mathbf{k} \mathbf{k}^{\prime}}\right|\right)\left(\int \frac{d \Omega_{\mathbf{k}}}{4 \pi} \frac{d \Omega_{\mathbf{k}^{\prime \prime}}}{4 \pi}\left|J_{i \mathbf{k} \mathbf{k}^{\prime \prime}}\right|\right) .
$$

\section{APPENDIX B: EFFECT OF COULOMB INTERACTIONS}

In this section, we will show explicitly that the presence of Coulomb interactions does not alter the results derived above. This is specific to our case of a continuum model with quadratic band dispersion and the assumption of exact particle hole symmetry [19]. When particle hole symmetry is broken, the Coulomb interaction will additionally screen the Higgs mode contribution to the nonlinear current.

We introduce long-range Coulomb interactions with potential $V_{q} \sim 1 / q^{2}$ and decouple the Coulomb action in the density channel by means of the Hubbard-Stratonovich transformation

$$
\begin{aligned}
& \exp \left(\int d \tau \sum_{\mathbf{q}}\left(\sum_{i \mathbf{k} \sigma} c_{i \mathbf{k}+\mathbf{q} \sigma}^{\dagger} c_{i \mathbf{k} \sigma}\right) V_{q}\left(\sum_{i \mathbf{k} \sigma} c_{i \mathbf{k}-\mathbf{q} \sigma}^{\dagger} c_{i \mathbf{k} \sigma}\right)\right) \\
& \quad=\int \mathcal{D} \rho(\mathbf{q}, \tau) \exp \left(-\int d \tau \sum_{\mathbf{q}}\left(\frac{1}{V_{q}} \rho(\mathbf{q}, \tau) \rho(-\mathbf{q}, \tau)+\sum_{i \mathbf{k} \sigma} \rho(\mathbf{q}, \tau) c_{i \mathbf{k}-\mathbf{q} \sigma}^{\dagger} c_{i \mathbf{k} \sigma}\right)\right) .
\end{aligned}
$$

In the presence of long-range Coulomb interactions, the phase action in Eq. (A17) is modified according to

$$
S_{\theta}=-\frac{1}{2} \sum_{\Omega_{n} \mathbf{q}}\left(\begin{array}{c}
\delta_{\mathbf{q}, 0} \frac{-i \Omega_{n}}{2} \theta_{1} \\
\delta_{\mathbf{q}, 0} \frac{-i \Omega_{n}}{2} \theta_{2} \\
\rho\left(-\Omega_{n},-\mathbf{q}\right)
\end{array}\right)\left(\begin{array}{ccc}
-\chi_{1}+\frac{\lambda}{\Omega_{n}^{2}} & -\frac{\lambda}{\Omega_{n}^{2}} & i \chi_{1} \\
-\frac{\lambda}{\Omega_{n}^{2}} & -\chi_{2}+\frac{\lambda}{\Omega_{n}^{2}} & i \chi_{2} \\
-i \chi_{1} & -i \chi_{2} & \frac{1}{\nabla_{q}}-\chi_{1}-\chi_{2}
\end{array}\right)\left(\begin{array}{c}
\delta_{\mathbf{q}, 0} \frac{i \Omega_{n}}{2} \theta_{1} \\
\delta_{\mathbf{q}, 0} \frac{i \Omega_{n}}{2} \theta_{2} \\
\rho\left(\Omega_{n}, \mathbf{q}\right)
\end{array}\right)
$$

Here, we have introduced the short notation $\chi_{i}$ for the susceptibility $\chi_{i}^{\sigma_{3} \sigma_{3}}$ defined in Eq. (A25). The light coupling term of Eq. (A18) becomes

$$
S_{\theta, A}=\sum_{i, \Omega_{m}} \frac{s_{i} e^{2}}{2 m_{i}} A^{2}\left(\Omega_{m}\right) \chi_{i}\left(\rho\left(-\Omega_{m}\right)-i \frac{-i \Omega_{n}}{2} \theta_{i}\left(-\Omega_{n}\right)\right) .
$$

The matrix in Eq. (B2) has a singular eigenvalue corresponding to the eigenvector $(i, i, 1)$. We can therefore reduce the description to

$$
S_{\theta}=-\frac{1}{2} \sum_{\Omega_{n}}\left(\begin{array}{c}
\frac{-i \Omega_{n}}{2} \theta_{L} \\
\rho-i \frac{-i \Omega_{n}}{2} \theta_{G}
\end{array}\right)\left(\begin{array}{cc}
-\chi_{1}-\chi_{2}+\frac{4 \lambda}{\Omega_{n}^{2}} & -\chi_{1}+\chi_{2} \\
-\chi_{1}+\chi_{2} & -\chi_{1}-\chi_{2}
\end{array}\right)\left(\begin{array}{c}
\frac{i \Omega_{n}}{2} \theta_{L} \\
\rho+i \frac{i \Omega_{n}}{2} \theta_{G}
\end{array}\right)
$$

where we have taken the limit $1 / V_{q}=0(q=0)$ and have defined new variables

$$
\begin{gathered}
\theta_{G}=\left(\theta_{1}+\theta_{2}\right) / 2, \\
\theta_{L}=\left(\theta_{1}-\theta_{2}\right) / 2 .
\end{gathered}
$$

The light-coupling term Eq. (B3) becomes

$$
S_{\theta, A}=\sum_{\Omega_{n}} \frac{e^{2} A^{2}}{4}\left(\begin{array}{l}
s_{1} \chi_{1} / m_{1}-s_{2} \chi_{2} / m_{2} \\
s_{1} \chi_{1} / m_{1}+s_{2} \chi_{2} / m_{2}
\end{array}\right)\left(\begin{array}{c}
\frac{-i \Omega_{n}}{2} \theta_{L} \\
\rho-i \frac{-i \Omega_{n}}{2} \theta_{G}
\end{array}\right)
$$



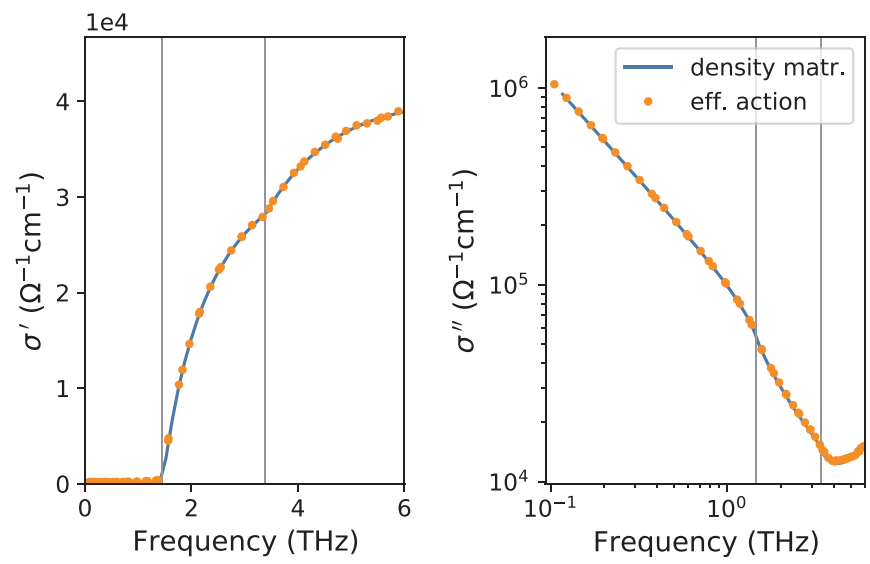

FIG. 16. Real and imaginary part of optical conductivity computed in the time-dependent density-matrix formalism (blue lines) and from diagrams Figs. 2(a) and 2(b) in the effective action approach. There is perfect agreement between the two methods.

and after Gaussian integration we obtain

$$
\tilde{S}_{\theta}=\sum_{\Omega_{n}} \frac{1}{2} \frac{e^{4} A^{4}}{4} \frac{\chi_{1} \chi_{2}\left(\chi_{1} / m_{1}^{2}+\chi_{2} / m_{2}^{2}\right) \Omega_{n}^{2}-\lambda\left(s_{1} \chi_{1} / m_{1}+s_{2} \chi_{2} / m_{2}\right)^{2}}{\lambda\left(\chi_{1}+\chi_{2}\right)-\chi_{1} \chi_{2} \Omega_{n}^{2}} .
$$

Together with the nonlinear diamagnetic density-fluctuation contribution we arrive at the Leggett action $\tilde{S}_{L}=S_{Q P \text {,dia }}^{(4)}+\tilde{S}_{\theta}$, which identically matches Eq. (A31) derived in the absence of Coulomb interactions.

\section{APPENDIX C: FIRST-ORDER CURRENTS AND OPTICAL CONDUCTIVITY}

The paramagnetic first-order current $\left.j_{P}\right|_{1}$ is represented by the diagram in Fig. 2(a) and explicitly given by a functional derivative of Eq. (A21). After analytical continuation and MB substitution one arrives at

$$
\left.j_{P}(-\omega)\right|_{1}=A(\omega) \sum_{i} \frac{v_{F_{i}}^{2}}{3 N_{i}(0)} \int d \epsilon d \epsilon^{\prime} W_{i}\left(\epsilon, \epsilon^{\prime}\right) \chi_{i}^{\sigma_{0} \sigma_{0}}\left(\omega, \epsilon, \epsilon^{\prime}\right) .
$$

The diamagnetic first-order current $\left.j_{P}\right|_{1}$ reads

$$
\left.j_{D}(-\omega)\right|_{1}=-A(-\omega) \sum_{i} \frac{e^{2}}{m_{i}} s_{i} \sum_{\mathbf{k} \sigma}\left\langle c_{i \mathbf{k} \sigma}^{\dagger} c_{i \mathbf{k} \sigma}\right\rangle,
$$

where we have used that $\frac{\delta}{\delta A(\omega)} A^{2}(0)=\frac{\delta}{\delta A(\omega)} \int d \omega^{\prime} A\left(-\omega^{\prime}\right) A\left(\omega^{\prime}\right)=2 A(-\omega)$. Note that the $\mathbf{k}$ sum does not vanish away from the Fermi surface and therefore strongly depends on the numerical cutoff. Here, we follow Murotani [20] and regularize the integral as

$$
\left.j_{D}(-\omega)\right|_{1}=A(\omega) \sum_{i} \frac{e^{2} n_{i}}{m_{i}} \int d \epsilon d \epsilon^{\prime} \frac{f(\epsilon)-f\left(\epsilon^{\prime}\right)}{\epsilon-\epsilon^{\prime}} W_{i}\left(\epsilon, \epsilon^{\prime}\right)
$$

with and Fermi function $f(\epsilon)$ and the band specific carrier density $n_{i}=k_{F_{i}}^{3} / 3 \pi^{2}$. The diagrammatically derived linear order optical conductivity exactly matches the results computed in the time domain using the density matrix formalism, as shown in Fig. 16.

\section{APPENDIX D: THIRD-HARMONIC GENERATION}

For THG experiments, we are interested in the nonlinear current $j(3 \Omega)$ evaluated at $\omega=3 \Omega$ where $\Omega$ is the dominant frequency of the optical pulse $A(\Omega)$ :

$$
j(-3 \Omega)=-\left.\frac{\delta S[A]}{\delta A(\omega)}\right|_{\omega=3 \Omega} .
$$

A diagrammatic representation of Eq. (D1) is shown in Fig. 17. The field $A(\omega)$ with respect to which the functional derivative is performed is colored red. All four choices are equivalent. The functional derivative forces the external frequency of the field $A$ to be $3 \Omega$. In principal one now needs to integrate over all remaining external frequencies, while satisfying energy conservation. This can be numerically challenging. Here, we focus instead on the case of a monochromatic field $A(t)=A_{0} \cos \Omega t, A(\omega)=$ 
(a)

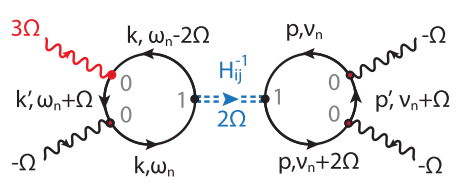

(c)

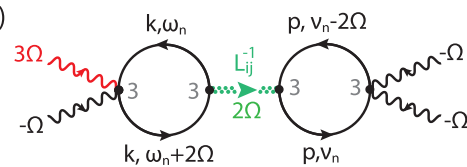

(b)

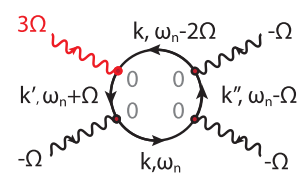

(d)

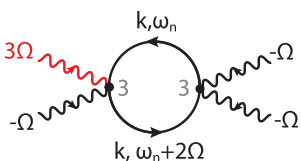

FIG. 17. Diagrammatic representation of THG signal. Red photon legs denote $A$ with respect to which the functional derivative has been performed. Higgs and Leggett propagators (double lines) correspond to an RPA summation shown in Fig. 3.

$\frac{A_{0}}{2}(\delta(\omega-\Omega)+\delta(\omega+\Omega))$ where external fields possess two discrete frequencies $\pm \Omega$. Then energy conservation dictates all remaining external legs to carry frequency $-\Omega$. Note that the energy flow through collective Higgs or Leggett propagators is $2 \Omega$, i.e., THG probes the optical kernel at twice the driving frequency as expected for a nonlinear process.

Figure 18 shows magnitude and phase of the Higgs contribution to the THG current $j_{H}(3 \Omega)$ as a function of $\Omega$ and $T$ for two interband couplings $v=0.05,0.4$. Panels (a), (b), (e), and (f) correspond to the limit of a dirty $\pi$ band and a clean $\sigma$ band, whereas the remaining panels are computed for two dirty bands. Both cases are possible descriptions of $\mathrm{MgB}_{2}$. Yellow spectral lines map out the Higgs resonance that follow $2 \Delta_{\pi}, 2 \Delta_{\sigma}$. In all cases the $\pi$ resonance is dominant, although the relative $\sigma$ contribution is enhanced in the dirty-dirty limit and for strong $v$. Increased interband coupling $v$ decreases and broadens the overall Higgs response.

The Higgs resonance is sharp at small $v$, but much broader in the $v=0.4$ case. Therefore, slices along the $T$ axis for a given drive frequency $\Omega$ do not exhibit a pronounced resonance peak. The observation of a resonance peak in Ref. [17] when experimentally sweeping the temperature would be therefore suggestive of a small $v$ coupling in $\mathrm{MgB}_{2}$. This is in disagreement to Refs. $[14,43]$ that experimentally determined a large $v$ based on evidence of the Leggett mode above $2 \Delta_{\pi}$.

Lower panels in Fig. 18 show a phase jump of $\pi$ in the THG current across the first Higgs resonance along the $\Omega$ direction that is most pronounced at low temperatures. The phase also shows features of the $\sigma$-Higgs resonance, albeit less clearly. Approaching the resonance along the $T$ axis does not yield a phase behavior that is consistently simple to interpret. These results are to be contrasted to the clean case where one expects a phase jump of $\pi / 2$.
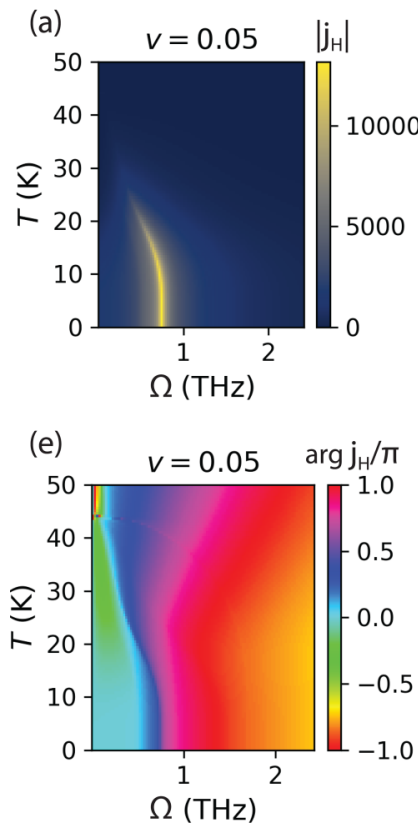

(b)

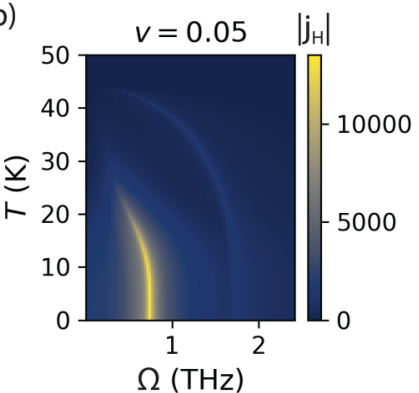

(f)

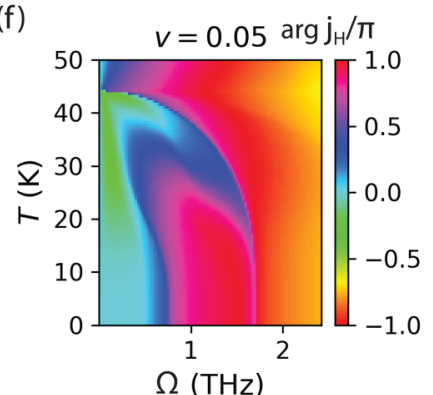

(c)

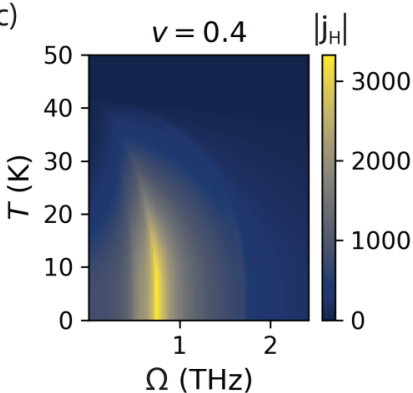

(g)

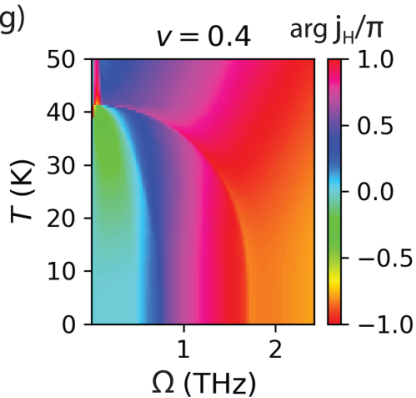

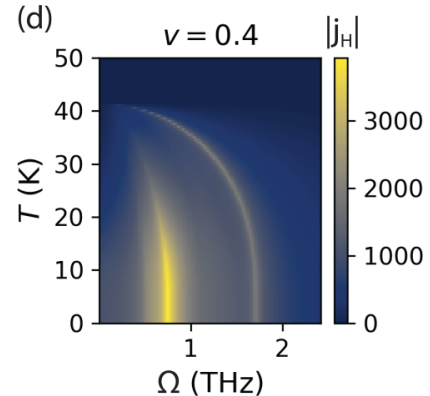

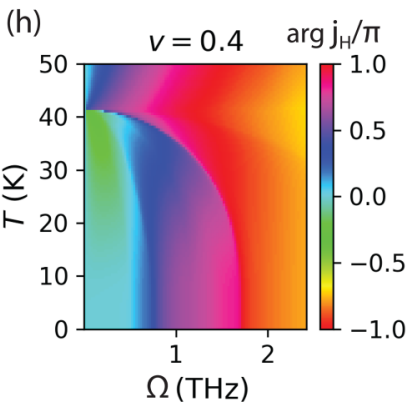

FIG. 18. Magnitude, up to a prefactor, [(a)-(d)] and phase [(e)-(h)] of Higgs contribution to THG current as a function of driving frequency $\Omega$ and temperature $T$. (a),(c),(e),(g) correspond to the dirty-clean case with $\gamma_{\pi}=100 \mathrm{meV}, \gamma_{\sigma}=0.01 \mathrm{meV}$ and [(c),(d),(g),(h)] correspond to the dirty-dirty case with $\gamma_{\pi}=100 \mathrm{meV}, \gamma_{\sigma}=50 \mathrm{meV}$. 

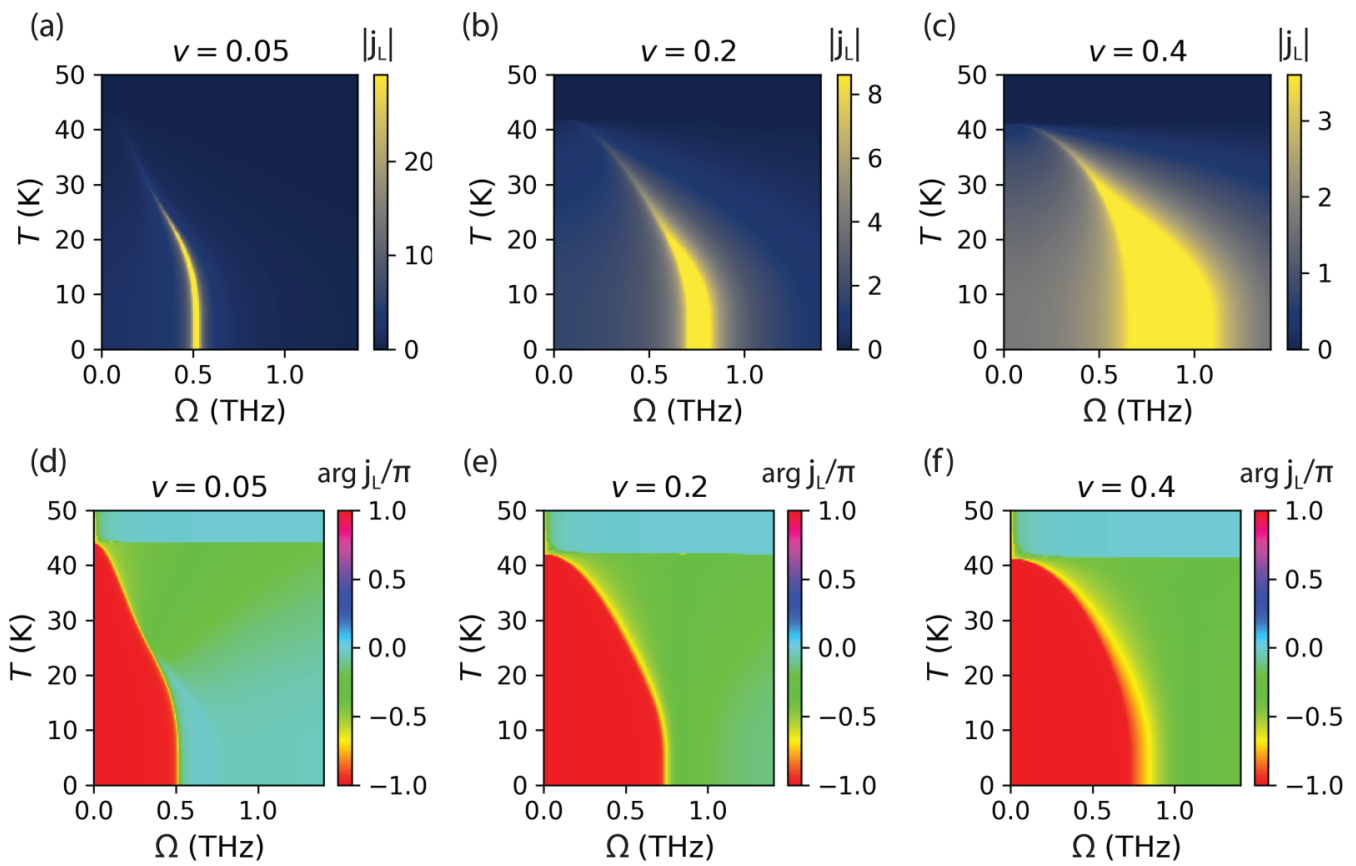

FIG. 19. Magnitude, up to a prefactor, [(a)-(c)] and phase [(d)-(f)] of Leggett contribution to THG current as a function of driving frequency $\Omega$ and temperature $T$ for various interband coupling parameters $v$ as denoted in plot titles.

Figure 19 shows the amplitude and phase response of the Leggett THG signal for three different coupling strengths $v=$ $0.02,0.2,0.5$. The overall contribution is about three magnitudes smaller than the Higgs contribution and therefore negligible. At large coupling, the Leggett resonance is very broad but sharpens at high temperatures. This observation was first reported in Ref. [42]. The phase shows a clear $\pi / 2$ jump across the resonance for all temperatures below $T_{C}$.

The density-fluctuation contribution is shown in Fig. 20 for $v=0.05$ in different impurity cases. Here clean refers to $\gamma=$ $0.01 \mathrm{meV}$ and dirty specifies $\gamma=100 \mathrm{meV}$. Results at different $v$ are nearly identical since the only $v$-dependent quantity in Eq. (A22) is the superconducting order parameter at finite $T$. For all impurity concentrations, the density-fluctuation mediated THG signal is peaked at the onset of the quasiparticle continuum of the $\pi$ band. The signal is about one order of magnitude smaller than the Higgs contribution in the small $v$ case. For $v=0.4$, the density-fluctuation signal remains nearly identical but the

(a)

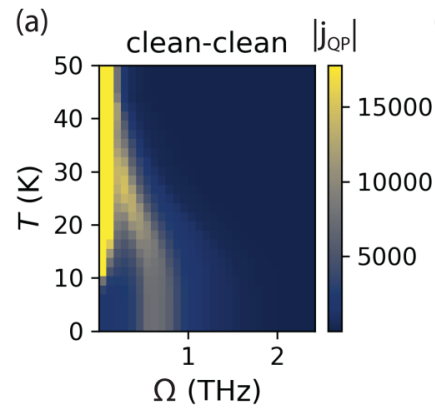

(e)

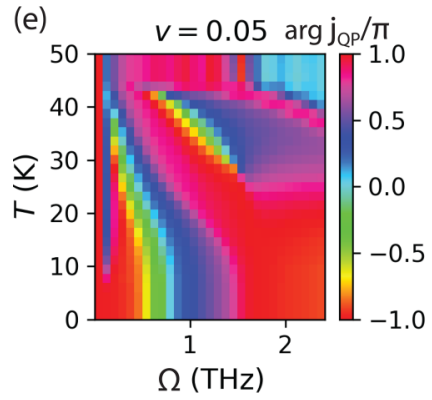

(b)

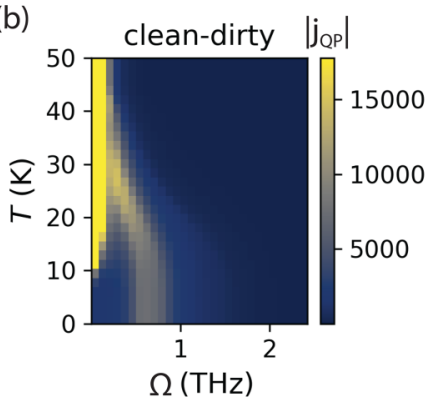

(f)

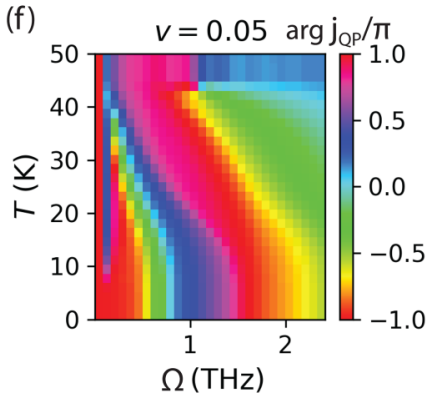

(c)

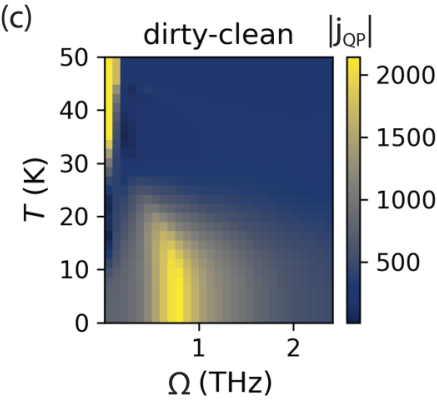

(g)

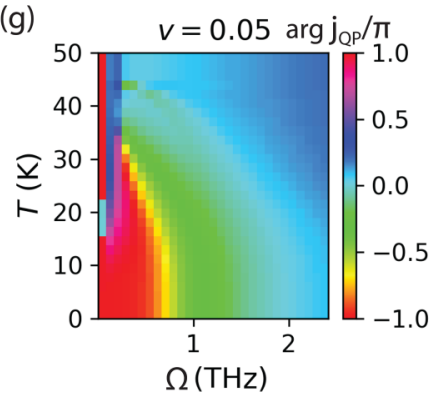

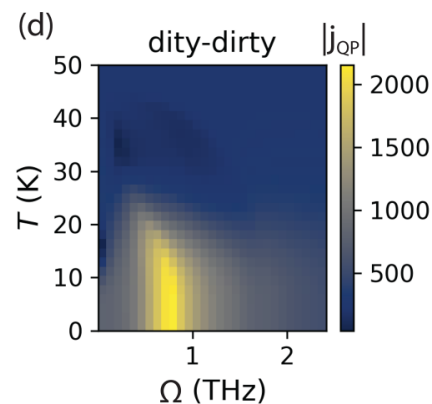

(h)

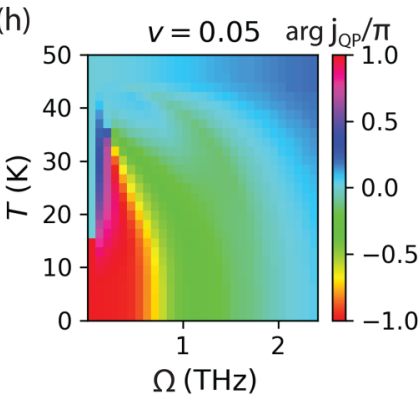

FIG. 20. Magnitude, up to a prefactor, [(a)-(d)] and phase [(e)-(h)] of density-fluctuation contribution to THG current as a function of driving frequency $\Omega$ and temperature $T$ for cases (a) clean-clean (b) clean-dirty case, (c) dirty-clean, and (d) dirty-dirty. 
Higgs signal increases, so that the Higgs contribution is only slightly larger. In all but the dirty-dirty case, the density-fluctuation signal has a large contribution for small $\Omega$ and large $T$.

The bottom row of Fig. 20 shows the phase of the non-linear THG signal. In the dirty-clean and dirty-dirty cases we observe a clear phase jump of $\pi / 2$ across a resonance.

\section{APPENDIX E: DENSITY-MATRIX EQUATION OF MOTION FORMULATION}

The following derivation uses notation similar to [20] and is included to make the present paper self-contained. We start from the mean field BCS Hamiltonian

$$
\mathcal{H}_{0}=\sum_{i \mathbf{k} \sigma} \epsilon_{i \mathbf{k}} c_{i \mathbf{k} \sigma}^{\dagger} c_{i \mathbf{k} \sigma}+\sum_{i \mathbf{k}}\left(\Delta_{i} c_{i-\mathbf{k} \uparrow}^{\dagger} c_{i \mathbf{k} \downarrow}^{\dagger}\right),
$$

where $\epsilon_{i \mathbf{k}}=s_{i}\left(\mathbf{k}^{2} / 2 m_{i}-\epsilon_{F_{i}}\right)$ and the superconducting order parameter is self-consistently determined by $\Delta_{i}=$ $\sum_{j \mathbf{k}} U_{i j}\left\langle c_{j-\mathbf{k} \downarrow} c_{j \mathbf{k} \uparrow}\right\rangle$. To incorporate the pulse, we add paramagnetic and diamagnetic coupling to the laser field through

$$
\mathcal{H}_{1}=-\sum_{i \mathbf{k} \mathbf{k}^{\prime} \sigma} \mathbf{J}_{i \mathbf{k} \mathbf{k}^{\prime}} \cdot \mathbf{A} c_{i \mathbf{k} \sigma}^{\dagger} c_{i \mathbf{k}^{\prime} \sigma}+\sum_{i \mathbf{k} \sigma} \frac{s_{i} e^{2}}{2 m_{i}} \mathbf{A}^{2} c_{i \mathbf{k} \sigma}^{\dagger} c_{i \mathbf{k} \sigma} .
$$

The full Hamiltonian is given by $\mathcal{H}=\mathcal{H}_{0}+\mathcal{H}_{1}$. The current density can be calculated using

$$
\mathbf{j}=-\left\langle\frac{\delta \mathcal{H}}{\delta \mathbf{A}}\right\rangle=\mathbf{j}_{P}+\mathbf{j}_{D}
$$

where the paramagnetic component $\mathbf{j}_{P}$ and diamagnetic component $\mathbf{j}_{D}$ correspond to the first and second terms in $\mathcal{H}_{1}$ respectively. The equilibrium Hamiltonian can be diagonalized through a Bogoliubov transformation, where we introduce the fermionic Bogoliubov quasiparticles in the form of the two-component spinor

$$
\left(\begin{array}{c}
\psi_{i \mathbf{k}}^{1} \\
\psi_{i \mathbf{k}}^{2}
\end{array}\right)=\left(\begin{array}{cc}
u_{i \mathbf{k}} & -v_{i \mathbf{k}} \\
v_{i \mathbf{k}}^{*} & u_{i \mathbf{k}}
\end{array}\right)\left(\begin{array}{c}
c_{i \mathbf{k} \uparrow} \\
c_{i(-\mathbf{k}) \downarrow}^{\dagger}
\end{array}\right),
$$

where

$$
E_{i k}=\sqrt{\epsilon_{i k}^{2}+\left|\Delta_{i}^{\mathrm{eq}}\right|^{2}}, \quad u_{i k}^{2}=\frac{1}{2}\left(1+\frac{\epsilon_{i k}}{E_{i k}}\right), \quad\left|v_{i k}\right|^{2}=\frac{1}{2}\left(1-\frac{\epsilon_{i k}}{E_{i k}}\right),
$$

and $\Delta_{i}^{\text {eq }}$ is the equilibrium value of the gap. We are free to choose the initial phase so that $u_{i \mathbf{k}}$ and $v_{i \mathbf{k}}$ are both real. Next, we construct the density matrix $\rho$ in the Bogoliubov quasiparticle basis

$$
\rho=\left|\psi_{0}\right\rangle\left\langle\psi_{0}\right|=\left(\begin{array}{cc}
\rho_{i \mathbf{k} \mathbf{k}^{\prime}}^{11} & \rho_{i \mathbf{k} \mathbf{k}^{\prime}}^{12} \\
\rho_{i \mathbf{k} \mathbf{k}^{\prime}}^{21} & \rho_{i \mathbf{k} \mathbf{k}^{\prime}}^{22}
\end{array}\right)=\left(\begin{array}{cc}
\left\langle\psi_{i \mathbf{k}}^{1 \dagger} \psi_{i \mathbf{k}^{\prime}}^{1}\right\rangle & \left\langle\psi_{i \mathbf{k}}^{1 \dagger} \psi_{i \mathbf{k}^{\prime}}^{2}\right\rangle \\
\left\langle\psi_{i \mathbf{k}}^{2 \dagger} \psi_{i \mathbf{k}^{\prime}}^{1}\right\rangle & \left\langle\psi_{i \mathbf{k}}^{2 \dagger} \psi_{i \mathbf{k}^{\prime}}^{2}\right\rangle
\end{array}\right)
$$

We can now rewrite the Hamiltonian in terms of the quasiparticles and their expectation values

$$
\mathcal{H}_{0}=\sum_{i \mathbf{k}}\left(\begin{array}{ll}
\psi_{i \mathbf{k}}^{1 \dagger} & \psi_{i \mathbf{k}}^{2 \dagger}
\end{array}\right)\left(\begin{array}{cc}
\epsilon_{i \mathbf{k}}\left(u_{i \mathbf{k}}^{2}-v_{i \mathbf{k}}^{2}\right)+u_{i \mathbf{k}} v_{i \mathbf{k}}\left(\Delta_{i}+\Delta_{i}^{*}\right) & 2 u_{i \mathbf{k}} v_{i \mathbf{k}} \epsilon_{i \mathbf{k}}+\Delta_{i}^{*} v_{i \mathbf{k}}^{2}-\Delta_{i} u_{i \mathbf{k}}^{2} \\
2 u_{i \mathbf{k}} v_{i \mathbf{k}} \epsilon_{i \mathbf{k}}+\Delta_{i} v_{i \mathbf{k}}^{2}-\Delta_{i}^{*} u_{i \mathbf{k}}^{2} & -\epsilon_{i \mathbf{k}}\left(u_{i \mathbf{k}}^{2}-v_{i \mathbf{k}}^{2}\right)-u_{i \mathbf{k}} v_{i \mathbf{k}}\left(\Delta_{i}+\Delta_{i}^{*}\right)
\end{array}\right)\left(\begin{array}{c}
\psi_{i \mathbf{k}}^{1} \\
\psi_{i \mathbf{k}}^{2}
\end{array}\right) .
$$

In equilibrium, where $\Delta_{i}=\Delta_{i}^{\text {eq }}$, this reduces to

$$
\mathcal{H}_{0}^{\mathrm{eq}}=\sum_{i \mathbf{k}}\left(\psi_{i \mathbf{k}}^{1 \dagger} \quad \psi_{i \mathbf{k}}^{2 \dagger}\right)\left(\begin{array}{cc}
E_{i \mathbf{k}} & 0 \\
0 & -E_{i \mathbf{k}}
\end{array}\right)\left(\begin{array}{l}
\psi_{i \mathbf{k}}^{1} \\
\psi_{i \mathbf{k}}^{2}
\end{array}\right) .
$$

In non-equilibrium, we can write the time-dependent gap as $\Delta_{i}(t)=\Delta_{i}^{\mathrm{eq}}+\delta \Delta_{i}(t)$. Furthermore we can decompose $\delta \Delta_{i}(t)=$ $\delta \Delta_{i}^{\prime}(t)+i \delta \Delta_{i}^{\prime \prime}(t)$ into real and imaginary parts. Then, we find

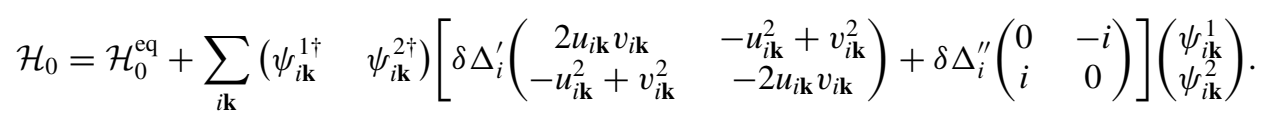

The coupling to the pulse becomes

$$
\begin{aligned}
\mathcal{H}_{1}= & -\sum_{i \mathbf{k} \mathbf{k}^{\prime}} \mathbf{J}_{i \mathbf{k} \mathbf{k}^{\prime}} \cdot \mathbf{A}\left(\begin{array}{ll}
\psi_{i \mathbf{k}}^{1 \dagger} & \psi_{i \mathbf{k}}^{2 \dagger}
\end{array}\right)\left(\begin{array}{cc}
l_{i \mathbf{k} \mathbf{k}^{\prime}} & -p_{i \mathbf{k} \mathbf{k}^{\prime}} \\
p_{i \mathbf{k} \mathbf{k}^{\prime}} & l_{i \mathbf{k} \mathbf{k}^{\prime}}
\end{array}\right)\left(\begin{array}{l}
\psi_{i \mathbf{k}^{\prime}}^{1} \\
\psi_{i \mathbf{k}^{\prime}}^{2}
\end{array}\right) \\
& +\sum_{i \mathbf{k}} s_{i} \frac{e^{2} \mathbf{A}^{2}}{2 m_{i} E_{i \mathbf{k}}}\left(\begin{array}{ll}
\psi_{i \mathbf{k}}^{1 \dagger} & \psi_{i \mathbf{k}}^{2 \dagger}
\end{array}\right)\left(\begin{array}{cc}
\epsilon_{i \mathbf{k}} & \Delta_{i}^{\mathrm{eq}} \\
\Delta_{i}^{\mathrm{eq}} & -\epsilon_{i \mathbf{k}}
\end{array}\right)\left(\begin{array}{l}
\psi_{i \mathbf{k}}^{1} \\
\psi_{i \mathbf{k}}^{2}
\end{array}\right),
\end{aligned}
$$


where

$$
\begin{aligned}
l_{i \mathbf{k} \mathbf{k}^{\prime}} & =u_{i \mathbf{k}} u_{i \mathbf{k}^{\prime}}+v_{i \mathbf{k}} v_{i \mathbf{k}^{\prime}}, \\
p_{i \mathbf{k} \mathbf{k}^{\prime}} & =v_{i \mathbf{k}} u_{i \mathbf{k}^{\prime}}-u_{i \mathbf{k}} v_{i \mathbf{k}^{\prime}}
\end{aligned}
$$

Notice the total Hamiltonian can be expressed as

$$
\mathcal{H}_{0}+\mathcal{H}_{1}=\sum_{a b} \sum_{i \mathbf{k} \mathbf{k}^{\prime}} \psi_{i \mathbf{k}}^{a \dagger} h_{i \mathbf{k} \mathbf{k}^{\prime}}^{a b} \psi_{i \mathbf{k}^{\prime}}^{b} .
$$

Next, we decompose the gap into real and imaginary parts $\Delta_{i}=\Delta_{i}^{\prime}+i \Delta_{i}^{\prime \prime}$. After the transformation, the gap equation becomes

$$
\begin{gathered}
\Delta_{i}^{\prime}=\sum_{j} U_{i j} \sum_{\mathbf{k}}\left[-u_{j \mathbf{k}} v_{j \mathbf{k}}\left(\rho_{j \mathbf{k} \mathbf{k}}^{11}-\rho_{j \mathbf{k} \mathbf{k}}^{22}\right)+\frac{1}{2}\left(u_{j \mathbf{k}}^{2}-v_{j \mathbf{k}}^{2}\right)\left(\rho_{j \mathbf{k} \mathbf{k}}^{21}+\rho_{j \mathbf{k k}}^{12}\right)\right], \\
\Delta_{i}^{\prime \prime}=\sum_{j} U_{i j} \sum_{\mathbf{k}} \frac{1}{2 i}\left(\rho_{j \mathbf{k k}}^{21}-\rho_{j \mathbf{k k}}^{12}\right) .
\end{gathered}
$$

Finally, we write the paramagnetic and diamagnetic current densities in terms of density matrices

$$
\begin{gathered}
\mathbf{j}_{P}=\sum_{i \mathbf{k} \mathbf{k}^{\prime}} \mathbf{J}_{i \mathbf{k} \mathbf{k}^{\prime}}\left[l_{i \mathbf{k} \mathbf{k}^{\prime}}\left(\rho_{i \mathbf{k} \mathbf{k}^{\prime}}^{11}+\rho_{i \mathbf{k} \mathbf{k}^{\prime}}^{22}\right)+p_{i \mathbf{k} \mathbf{k}^{\prime}}\left(\rho_{i \mathbf{k} \mathbf{k}^{\prime}}^{21}-\rho_{i \mathbf{k} \mathbf{k}^{\prime}}^{12}\right)\right] \\
\mathbf{j}_{D}=-\sum_{i \mathbf{k}} s_{i} \frac{e^{2} \mathbf{A}}{m_{i} E_{i \mathbf{k}}}\left[\left(u_{i \mathbf{k}}^{2}-v_{i \mathbf{k}}^{2}\right)\left(\rho_{i \mathbf{k k}}^{11}-\rho_{i \mathbf{k k}}^{22}\right)+2 u_{i \mathbf{k}} v_{i \mathbf{k}}\left(\rho_{i \mathbf{k k}}^{21}+\rho_{i \mathbf{k k}}^{12}\right)\right] .
\end{gathered}
$$

We use Heisenberg's equation of motion to find the time dependence of the density matrix. Writing the density matrix in vector form,

$$
\rho_{i \mathbf{k} \mathbf{k}^{\prime}}=\left(\begin{array}{c}
\rho_{i \mathbf{k} \mathbf{k}^{\prime}}^{11} \\
\rho_{i \mathbf{k} \mathbf{k}^{\prime}}^{12} \\
\rho_{i \mathbf{k} \mathbf{k}^{\prime}}^{21} \\
\rho_{i \mathbf{k} \mathbf{k}^{\prime}}^{22}
\end{array}\right)
$$

Heisenberg's equation of motion is stated in form of a Liouville equation

$$
i \partial_{t} \rho_{i \mathbf{k} \mathbf{k}^{\prime}}=\sum_{\mathbf{q}}\left[H_{i \mathbf{k}^{\prime} \mathbf{q}}^{(1)} \rho_{i \mathbf{k q}}-H_{i \mathbf{q} \mathbf{k}}^{(2)} \rho_{i \mathbf{q} \mathbf{k}^{\prime}}\right]
$$

where we have defined the following two matrices:

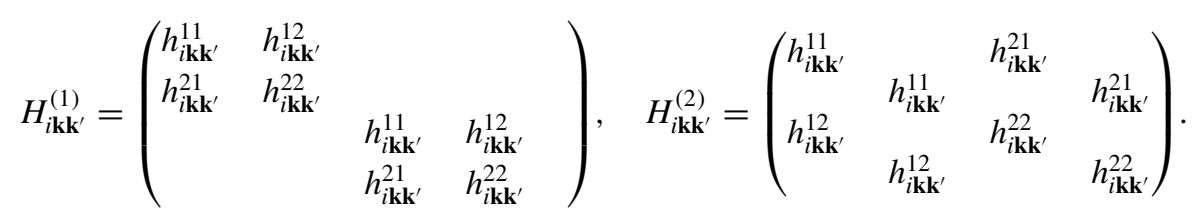

Note that we work in natural units where $\hbar=1$.

In order to solve the equation of motion with the impurity scattering replacement, we proceed using a perturbative approach with respect to $\mathbf{A}$. This involves expanding all relevant quantities in powers of $\mathbf{A}$. For instance, we expand $\rho_{i \mathbf{k k}^{\prime}}$ as $\rho_{i \mathbf{k} \mathbf{k}^{\prime}}=$ $\left.\rho_{i \mathbf{k} \mathbf{k}^{\prime}}\right|_{0}+\left.\rho_{i \mathbf{k} \mathbf{k}^{\prime}}\right|_{1}+\left.\rho_{i \mathbf{k} \mathbf{k}^{\prime}}\right|_{2}+\ldots$, where $\left.\rho_{i \mathbf{k} \mathbf{k}^{\prime}}\right|_{1}$ is proportional to $\mathbf{A},\left.\rho_{i \mathbf{k} \mathbf{k}^{\prime}}\right|_{2}$ is proportional to $\mathbf{A}^{2}$, etc.

We now solve each order separately. The zeroth-order components are simply the equilibrium values, where the quasiparticles are occupied according to Fermi statistics

$$
\begin{aligned}
& \left.\rho_{i \mathbf{k} \mathbf{k}^{\prime}}\right|_{0}=\delta_{k k^{\prime}}\left(\begin{array}{c}
f_{i \mathbf{k}} \\
0 \\
0 \\
1-f_{i \mathbf{k}}
\end{array}\right) \\
& \left.H_{i \mathbf{k} \mathbf{k}^{\prime}}^{(1)}\right|_{0}=\delta_{\mathbf{k} \mathbf{k}^{\prime}} E_{i \mathbf{k}}\left(\begin{array}{cccc}
1 & & & \\
& -1 & & \\
& & 1 & \\
& & & -1
\end{array}\right),\left.\quad H_{i \mathbf{k} \mathbf{k}^{\prime}}^{(2)}\right|_{0}=\delta_{\mathbf{k k}^{\prime}} E_{i \mathbf{k}}\left(\begin{array}{cccc}
1 & & & \\
& 1 & & \\
& & -1 & \\
& & & -1
\end{array}\right) \text {. }
\end{aligned}
$$


We can also use these values to calculate the equilibrium value of the gap, exchanging the sum in the gap equation with an integral over the energy:

$$
\Delta_{i}^{\mathrm{eq}}=\sum_{j} U_{i j} N_{j}(0) \Delta_{j}^{\mathrm{eq}} \int_{-\omega_{d}}^{\omega_{d}} \frac{d \epsilon}{2 \sqrt{\epsilon^{2}+\left(\Delta_{i}^{\mathrm{eq}}\right)^{2}}} \tanh \left(\frac{\beta}{2} \sqrt{\epsilon^{2}+\left(\Delta_{i}^{\mathrm{eq}}\right)^{2}}\right) .
$$

Now we proceed with the first order. Considering only the terms proportional to $\mathbf{A}$, the equation of motion becomes

$$
\left.i \partial_{t} \rho_{i \mathbf{k} \mathbf{k}^{\prime}}\right|_{1}=\left.\left(\left.H_{i \mathbf{k}^{\prime} \mathbf{k}^{\prime}}^{(1)}\right|_{0}-\left.H_{i \mathbf{k} \mathbf{k}}^{(2)}\right|_{0}\right) \rho_{i \mathbf{k} \mathbf{k}^{\prime}}\right|_{1}+\left(\left.\left.H_{i \mathbf{k}^{\prime} \mathbf{k}}^{(1)}\right|_{1} \rho_{i \mathbf{k} \mathbf{k}}\right|_{0}-\left.\left.H_{i \mathbf{k}^{\prime} \mathbf{k}}^{(2)}\right|_{1} \rho_{i \mathbf{k}^{\prime} \mathbf{k}^{\prime}}\right|_{0}\right)
$$

where

$$
\begin{array}{r}
\left.H_{i \mathbf{k} \mathbf{k}^{\prime}}^{(1)}\right|_{1}=-\mathbf{J}_{i \mathbf{k} \mathbf{k}^{\prime}} \cdot \mathbf{A}\left(\begin{array}{cccc}
l_{i \mathbf{k} \mathbf{k}^{\prime}} & -p_{i \mathbf{k} \mathbf{k}^{\prime}} & & \\
p_{i \mathbf{k} \mathbf{k}^{\prime}} & l_{i \mathbf{k} \mathbf{k}^{\prime}} & & \\
& & l_{i \mathbf{k} \mathbf{k}^{\prime}} & -p_{i \mathbf{k} \mathbf{k}^{\prime}} \\
& & p_{i \mathbf{k} \mathbf{k}^{\prime}} & l_{i \mathbf{k} \mathbf{k}^{\prime}}
\end{array}\right), \\
\left.H_{i \mathbf{k} \mathbf{k}^{\prime}}^{(2)}\right|_{1}=-\mathbf{J}_{i \mathbf{k} \mathbf{k}^{\prime}} \cdot \mathbf{A}\left(\begin{array}{cccc}
l_{i \mathbf{k} \mathbf{k}^{\prime}} & & p_{i \mathbf{k} \mathbf{k}^{\prime}} & \\
-p_{i \mathbf{k} \mathbf{k}^{\prime}} & l_{i \mathbf{k} \mathbf{k}^{\prime}} & l_{i \mathbf{k} \mathbf{k}^{\prime}} & p_{i \mathbf{k} \mathbf{k}^{\prime}} \\
& -p_{i \mathbf{k} \mathbf{k}^{\prime}} & & l_{i \mathbf{k} \mathbf{k}^{\prime}}
\end{array}\right) .
\end{array}
$$

We solve the equation by writing it in terms of new functions $F_{i \mathbf{k} \mathbf{k}^{\prime}}^{a b}$. The first-order expression of $\rho_{i \mathbf{k} \mathbf{k}^{\prime}}$ becomes

$$
\left.\rho_{i \mathbf{k} \mathbf{k}^{\prime}}\right|_{1}=\mathbf{J}_{i \mathbf{k}^{\prime} \mathbf{k}} \cdot \mathbf{e}\left(\begin{array}{c}
l_{i \mathbf{k} \mathbf{k}^{\prime}} F_{i \mathbf{k} \mathbf{k}^{\prime}}^{11} \\
p_{i \mathbf{k} \mathbf{k}^{\prime}} F_{i \mathbf{k} \mathbf{k}^{\prime}} \\
-p_{i \mathbf{k} \mathbf{k}^{\prime}} F_{i \mathbf{k} \mathbf{k}^{\prime}}^{21} \\
l_{i \mathbf{k} \mathbf{k}^{\prime}} F_{i \mathbf{k k}^{\prime}}^{22}
\end{array}\right),
$$

where $F_{i}^{a b}$ is defined according to

$$
\begin{gathered}
{\left[i \hbar \frac{\partial}{\partial t}-\left(E^{\prime}-E\right)\right] F_{i}^{11}\left(\epsilon, \epsilon^{\prime}\right)=\left(f^{\prime}-f\right) A,} \\
{\left[i \hbar \frac{\partial}{\partial t}-\left(E^{\prime}+E\right)\right] F_{i}^{21}\left(\epsilon, \epsilon^{\prime}\right)=-\left(1-f-f^{\prime}\right) A,} \\
F_{i}^{12}\left(\epsilon, \epsilon^{\prime}\right)=F_{i}^{21}\left(\epsilon, \epsilon^{\prime}\right)^{*} \\
F_{i}^{22}\left(\epsilon, \epsilon^{\prime}\right)=F_{i}^{11}\left(\epsilon, \epsilon^{\prime}\right)^{*} .
\end{gathered}
$$

We have introduced a simplified notation $\epsilon=\epsilon_{i \mathbf{k}}, E^{\prime}=E_{i \mathbf{k}^{\prime}}, F_{i \mathbf{k} \mathbf{k}^{\prime}}^{a b}=F_{i}^{a b}\left(\epsilon, \epsilon^{\prime}\right)$ etc. The collective modes do not couple linearly to light, and correspondingly $\left.\delta \Delta_{i}\right|_{1}=0$. This can be confirmed by using Eq. (E26) in the gap equation. Hence, we only examine the response of the current. By substituting Eq. (E26) into Eq. (E15), changing the momentum sums into integrals over energy, and making the Mattis-Bardeen replacement (5), we find

$$
\left.\mathbf{j}_{P}\right|_{1}=\mathbf{e} \sum_{i} \frac{e^{2} n_{i}}{m_{i}} \int d \epsilon d \epsilon^{\prime} W_{i}\left(\epsilon, \epsilon^{\prime}\right)\left[l_{i}\left(\epsilon, \epsilon^{\prime}\right)^{2} \operatorname{Re} F_{i}^{11}\left(\epsilon, \epsilon^{\prime}\right)+p_{i}\left(\epsilon, \epsilon^{\prime}\right)^{2} \operatorname{Re} F_{i}^{21}\left(\epsilon, \epsilon^{\prime}\right)\right] .
$$

We can also derive the induced diamagnetic current,

$$
\left.\mathbf{j}_{\mathrm{D}}(t)\right|_{1}=-\mathbf{A} \sum_{i} \frac{e^{2} n_{i}}{m_{i}}
$$

This derivation is carefully discussed in [20].

We now consider the second-order solution. Keeping only terms proportional to $\mathbf{A}$, we find that the off-diagonal terms in the equation of motion vanish, and as a result (E18) becomes

$$
i \partial_{t} \rho_{i \mathbf{k k}}=\left.\left.\left(H_{i \mathbf{k k}}^{(1)}-H_{i \mathbf{k k}}^{(2)}\right)\right|_{0} \rho_{i \mathbf{k k}}\right|_{2}+\sum_{\mathbf{q}}\left(\left.\left.H_{i \mathbf{k q}}^{(1)}\right|_{1} \rho_{i \mathbf{k q}}\right|_{1}-\left.\left.H_{i \mathbf{q} \mathbf{k}}^{(2)}\right|_{1} \rho_{i \mathbf{q} \mathbf{k}}\right|_{1}\right)+\left.\left.\left(H_{i \mathbf{k k}}^{(1)}-H_{i \mathbf{k k} \mathbf{k}}^{(2)}\right)\right|_{2} \rho_{i \mathbf{k k}}\right|_{0} .
$$

We can decompose $\left.H_{i \mathbf{k k}}^{1}\right|_{2}$ and $\left.H_{i \mathbf{k k}}^{2}\right|_{2}$ into contributions from the diamagnetic current mediated by density fluctuations, Higgs mode, and Leggett mode as follows:

$$
\left.H_{i \mathbf{k k}}^{(1,2)}\right|_{2}=\left.H_{i \mathbf{k} \mathbf{k}}^{(1,2)}\right|_{2, D}+\left.H_{i \mathbf{k k}}^{(1,2)}\right|_{2, H}+\left.H_{i \mathbf{k k}}^{(1,2)}\right|_{2, L}
$$


where the diamagnetic density-fluctuation current contribution is

$$
\left.H_{i \mathbf{k} \mathbf{k}^{\prime}}^{(1)}\right|_{2, D}=\delta_{\mathbf{k} \mathbf{k}^{\prime}} \frac{s_{i} e^{2}}{2 m_{i} E_{i \mathbf{k}}} \mathbf{A}^{2}\left(\begin{array}{cccc}
\epsilon_{i \mathbf{k}} & \Delta_{i} & & \\
\Delta_{i} & -\epsilon_{i \mathbf{k}} & & \\
& & \epsilon_{i \mathbf{k}} & \Delta_{i} \\
& & \Delta_{i} & -\epsilon_{i \mathbf{k}}
\end{array}\right),\left.\quad H_{i \mathbf{k} \mathbf{k}^{\prime}}^{(2)}\right|_{2, D}=\delta_{\mathbf{k} \mathbf{k}^{\prime}} \frac{s_{i} e^{2}}{2 m_{i}} \mathbf{A}^{2}\left(\begin{array}{cccc}
\epsilon_{i \mathbf{k}} & & \Delta_{i} & \\
& \epsilon_{i \mathbf{k}} & & \Delta_{i} \\
\Delta_{i} & & -\epsilon_{i \mathbf{k}} & \\
& \Delta_{i} & & -\epsilon_{i \mathbf{k}}
\end{array}\right),
$$

the Higgs contribution is

$$
\left.H_{i \mathbf{k} \mathbf{k}^{\prime}}^{(1)}\right|_{2, H}=\delta_{\mathbf{k} \mathbf{k}^{\prime}} \frac{\left.\delta \Delta_{i}^{\prime}\right|_{2}}{E_{i \mathbf{k}}}\left(\begin{array}{cccc}
\Delta_{i} & -\epsilon_{i \mathbf{k}} & & \\
-\epsilon_{i \mathbf{k}} & -\Delta_{i} & & \\
& & \Delta_{i} & -\epsilon_{i \mathbf{k}} \\
& & -\epsilon_{i \mathbf{k}} & -\Delta_{i}
\end{array}\right),\left.\quad H_{i \mathbf{k} \mathbf{k}^{\prime}}^{(2)}\right|_{2, H}=\left.\delta_{\mathbf{k} \mathbf{k}^{\prime}} \delta \Delta_{i}^{\prime}\right|_{2}\left(\begin{array}{cccc}
\Delta_{i} & & -\epsilon_{i \mathbf{k}} & \\
& \Delta_{i} & & -\epsilon_{i \mathbf{k}} \\
-\epsilon_{i \mathbf{k}} & & -\Delta_{i} & \\
& -\epsilon_{i \mathbf{k}} & & -\Delta_{i}
\end{array}\right),
$$

and the Leggett contribution is

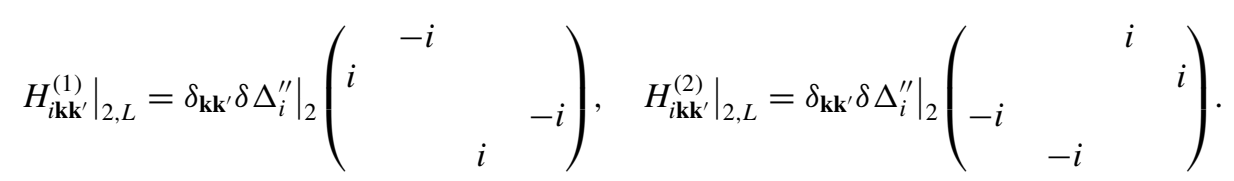

To simplify the equation of motion, we introduce a new angle-averaged quantity $r_{i}^{a b}(\epsilon)$ defined by

$$
r_{i}^{a b}(\epsilon)=\left.\int \frac{d \Omega_{\mathbf{k}}}{4 \pi} \rho_{i \mathbf{k k}}^{a b}\right|_{2} .
$$

The equations of motion can then be written as

$$
\begin{gathered}
i \partial_{t} r_{i}^{11}(\epsilon)=-2 i A \frac{\left(e v_{F i}\right)^{2}}{3} \int d \epsilon^{\prime}\left[l_{i}\left(\epsilon, \epsilon^{\prime}\right)^{2} \operatorname{Im} F_{i}^{11}\left(\epsilon, \epsilon^{\prime}\right)-p_{i}\left(\epsilon, \epsilon^{\prime}\right)^{2} \operatorname{Im} F_{i}^{21}\left(\epsilon, \epsilon^{\prime}\right)\right] W_{i}\left(\epsilon, \epsilon^{\prime}\right), \\
r_{i}^{22}(\epsilon)=-r_{i}^{11}(\epsilon)
\end{gathered}
$$

The terms $r_{i}^{11}$ and $r_{i}^{22}$ correspond to density fluctuations. The remaining $r_{i}^{a b}$ terms correspond to the collective modes. We break up the remaining terms into odd and even components, which are responsible for the Higgs and Leggett modes, respectively:

$$
\begin{gathered}
r_{i}^{21}(\epsilon)=r_{i}^{21, \text { odd }}(\epsilon)+r_{i}^{21, \text { even }}(\epsilon), \\
r_{i}^{21, \text { odd }}(-\epsilon)=-r_{i}^{21, \text { odd }}(\epsilon), \\
r_{i}^{21, \text { even }}(\epsilon)=r_{i}^{21, \text { even }}(-\epsilon) .
\end{gathered}
$$

The equations of motion for these terms are

$$
\begin{gathered}
{\left[i \partial_{t}-2 E\right] r_{i}^{21, \mathrm{odd}}(\epsilon)=-\left.(1-2 f)\left(u_{i}(\epsilon)^{2}-v_{i}(\epsilon)^{2}\right) \delta \Delta_{i}^{\prime}\right|_{2}-2 A \frac{\left(e v_{F i}\right)^{2}}{3} \int d \epsilon^{\prime} W_{i}\left(\epsilon, \epsilon^{\prime}\right) l_{i}\left(\epsilon, \epsilon^{\prime}\right) p_{i}\left(\epsilon, \epsilon^{\prime}\right)\left[F_{i}^{21}\left(\epsilon, \epsilon^{\prime}\right)-F_{i}^{22}\left(\epsilon, \epsilon^{\prime}\right)\right]} \\
{\left[i \partial_{t}-2 E\right] r_{i}^{21, \mathrm{even}}(\epsilon)=(1-2 f) \frac{\Delta_{i}^{\mathrm{eq}} s_{i} e^{2} \mathbf{A}^{2}}{2 m_{i} E}-\left.i(1-2 f) \delta \Delta_{i}^{\prime \prime}\right|_{2}}
\end{gathered}
$$

After exchanging momentum sums into energy integrals, the gap equations written in terms of the angle averaged quantities become

$$
\begin{gathered}
\left.\delta \Delta_{i}^{\prime}\right|_{2}=\sum_{j} U_{i j} N_{j}(0) \int d \epsilon\left\{-u_{j}(\epsilon) v_{j}(\epsilon)\left(r_{j}^{11}(\epsilon)-r_{j}^{22}(\epsilon)\right)+\frac{1}{2}\left(u_{j}(\epsilon)^{2}-v_{j}(\epsilon)^{2}\right)\left(r_{j}^{21}(\epsilon)+r_{j}^{12}(\epsilon)\right)\right\}, \\
\left.\delta \Delta_{i}^{\prime \prime}\right|_{2}=\sum_{j} U_{i j} N_{j}(0) \int d \epsilon \frac{1}{2 i}\left(r_{j}^{21}(\epsilon)-r_{j}^{12}(\epsilon)\right) .
\end{gathered}
$$

The equations of motion are solved numerically, and must be solved self-consistently with the gap equations at each time step. This condition induces the collective modes.

Finally, to consider pump-probe simulations and THG, we must go to third order. First, the diamagnetic third-order current can be directly calculated from the angle-averaged quantities $r_{i}^{a b}$.

$$
\mathbf{j}_{D}=-\sum_{i} \int d \epsilon \frac{s_{i} e^{2} \mathbf{A}}{m_{i}}\left[\left(u_{i}(\epsilon)^{2}-v_{i}(\epsilon)^{2}\right)\left(r_{i}^{11}(\epsilon)-r_{i}^{22}(\epsilon)\right)+2 u(\epsilon)_{i} v(\epsilon)_{i}\left(r_{i}^{21}(\epsilon)+r_{i}^{12}(\epsilon)\right)\right] .
$$


To find the paramagnetic third-order current, we start from the third-order equation of motion

$$
\left.i \partial_{t} \rho_{i \mathbf{k} \mathbf{k}^{\prime}}\right|_{3}=\left.\left.\left(H_{i \mathbf{k}^{\prime} \mathbf{k}^{\prime}}^{(1)}-H_{i \mathbf{k} \mathbf{k}}^{(2)}\right)\right|_{0} \rho_{i \mathbf{k} \mathbf{k}^{\prime}}\right|_{3}+\sum_{\mathbf{q}}\left(\left.\left.H_{i \mathbf{k}^{\prime} \mathbf{q}}^{(1)}\right|_{1} \rho_{i \mathbf{k q}}\right|_{2}-\left.\left.H_{i \mathbf{q} \mathbf{k}}^{(2)}\right|_{1} \rho_{i \mathbf{q} \mathbf{k}^{\prime}}\right|_{2}\right)+\left.\left.\left(H_{i \mathbf{k}^{\prime} \mathbf{k}^{\prime}}^{(1)}-H_{i \mathbf{k} \mathbf{k}}^{(2)}\right)\right|_{2} \rho_{i \mathbf{k} \mathbf{k}^{\prime}}\right|_{1} .
$$

We proceed by computing the equation explicitly for $\rho_{i \mathbf{k}^{\prime}}^{11}$. Here, we do not consider any contributions from the Leggett mode (i.e. terms involving $\delta \Delta_{i}^{\prime \prime}$ ) or the $\mathbf{A}^{2}$ part of the EM field. These terms vanish because of particle-hole symmetry. The remaining contributions, consisting of density fluctuations and Higgs mode, are

$$
\begin{aligned}
{\left.\left[i \partial_{t}-\left(E_{i \mathbf{k}^{\prime}}-E_{i \mathbf{k}}\right)\right] \rho_{i \mathbf{k} \mathbf{k}^{\prime}}^{11}\right|_{3}=} & \mathbf{J}_{i \mathbf{k}^{\prime} \mathbf{k}} \cdot \mathbf{e} l_{i \mathbf{k}^{\prime} \mathbf{k}}\left\{A\left[\left.\rho_{i \mathbf{k}^{\prime} \mathbf{k}^{\prime}}^{11}\right|_{2}-\left.\rho_{i \mathbf{k} \mathbf{k}}^{11}\right|_{2}\right]+\left.\delta \Delta_{i}^{\prime}\right|_{2}\left(\frac{\Delta_{i}}{E_{i \mathbf{k}^{\prime}}}-\frac{\Delta_{i}}{E_{i \mathbf{k}}}\right) F_{i \mathbf{k} \mathbf{k}^{\prime}}^{11}\right\} \\
& +\mathbf{J}_{i \mathbf{k}^{\prime} \mathbf{k}} \cdot \mathbf{e} p_{i \mathbf{k}^{\prime} \mathbf{k}}\left\{A\left[\left.\rho_{i \mathbf{k}^{\prime} \mathbf{k}^{\prime}}\right|_{2}+\left.\rho_{i \mathbf{k} \mathbf{k}}^{12}\right|_{2}\right]+\left.\delta \Delta_{i}^{\prime}\right|_{2}\left(-\frac{\epsilon_{i \mathbf{k}^{\prime}}}{E_{i \mathbf{k}}} F_{i \mathbf{k} \mathbf{k}^{\prime}}^{12}-\frac{\epsilon_{i \mathbf{k}}}{E_{i \mathbf{k}}} F_{i \mathbf{k} \mathbf{k}^{\prime}}^{21}\right)\right\} .
\end{aligned}
$$

The next step is to insert this into the expression for the paramagnetic current (E15). Replacing summation over $\mathbf{k}$ with integrals over energy, we find

$$
\begin{aligned}
\left.\mathbf{j}_{P}\right|_{3}= & \mathbf{e} \sum_{i \mathbf{k} \mathbf{k}^{\prime}} \mathbf{e} \cdot \mathbf{J}_{i \mathbf{k} \mathbf{k}^{\prime}}\left[l_{i \mathbf{k} \mathbf{k}^{\prime}}\left(\left.\rho_{i \mathbf{k} \mathbf{k}^{\prime}}^{11}\right|_{3}+\left.\rho_{i \mathbf{k} \mathbf{k}^{\prime}}^{22}\right|_{3}\right)+p_{i \mathbf{k} \mathbf{k}^{\prime}}\left(\left.\rho_{i \mathbf{k} \mathbf{k}^{\prime}}^{21}\right|_{3}-\left.\rho_{i \mathbf{k} \mathbf{k}^{\prime}}^{12}\right|_{3}\right)\right] \\
= & \mathbf{e} \sum_{i} N_{i}(0) \int d \epsilon d \epsilon^{\prime}\left[l_{i}\left(\epsilon, \epsilon^{\prime}\right)\left(\left.\left\langle\mathbf{e} \cdot \mathbf{J}_{i \mathbf{k} \mathbf{k}^{\prime}} \rho_{i \mathbf{k} \mathbf{k}^{\prime}}^{11}\right\rangle_{\mathrm{Av}}\right|_{3}+\left.\left\langle\mathbf{e} \cdot \mathbf{J}_{i \mathbf{k} \mathbf{k}^{\prime}} \rho_{i \mathbf{k} \mathbf{k}^{\prime}}^{22}\right\rangle_{\mathrm{Av}}\right|_{3}\right)\right. \\
& \left.+p_{i}\left(\epsilon, \epsilon^{\prime}\right)\left(\left.\left\langle\mathbf{e} \cdot \mathbf{J}_{i \mathbf{k} \mathbf{k}^{\prime}} \rho_{i \mathbf{k} \mathbf{k}^{\prime}}^{21}\right\rangle_{\mathrm{Av}}\right|_{3}-\left.\left\langle\mathbf{e} \cdot \mathbf{J}_{i \mathbf{k} \mathbf{k}^{\prime}} \rho_{i \mathbf{k} \mathbf{k}^{\prime}}^{12}\right\rangle_{\mathrm{Av}}\right|_{3}\right)\right]
\end{aligned}
$$

We see that in fact only the angle averaged quantities

$$
\left\langle\mathbf{e} \cdot \mathbf{J}_{i \mathbf{k} \mathbf{k}^{\prime}} \rho_{i \mathbf{k} \mathbf{k}^{\prime}}^{a b}\right\rangle_{\mathrm{Av}}=\int \frac{d \Omega_{\mathbf{k}}}{4 \pi} \frac{d \Omega_{\mathbf{k}^{\prime}}}{4 \pi} \mathbf{e} \cdot \mathbf{J}_{i \mathbf{k} \mathbf{k}^{\prime}} \rho_{i \mathbf{k} \mathbf{k}^{\prime}}^{a b}
$$

occur. The differential equation for these quantities is

$$
\begin{aligned}
{\left.\left[i \partial_{t}-\left(E^{\prime}-E\right)\right]\left\langle\mathbf{e} \cdot \mathbf{J}_{i \mathbf{k} \mathbf{k}^{\prime}} \rho_{i \mathbf{k} \mathbf{k}^{\prime}}^{11}\right\rangle_{\mathrm{Av}}\right|_{3}=} & \left\langle\left.\left|\mathbf{J}_{i \mathbf{k}^{\prime} \mathbf{k}} \cdot \mathbf{e}\right|^{2} l_{i \mathbf{k}^{\prime} \mathbf{k}}\left\{A\left[\left.\rho_{i \mathbf{k}^{\prime} \mathbf{k}^{\prime}}^{11}\right|_{2}-\left.\rho_{i \mathbf{k} \mathbf{k}}^{11}\right|_{2}\right]+\left.\delta \Delta_{i}^{\prime}\right|_{2}\left(\frac{\Delta_{i}}{E_{i \mathbf{k}^{\prime}}}-\frac{\Delta_{i}}{E_{i \mathbf{k}}}\right) F_{i \mathbf{k} \mathbf{k}^{\prime}}^{11}\right\}\right|_{\mathrm{Av}}\right. \\
& +\left\langle\left|\mathbf{J}_{i \mathbf{k}^{\prime} \mathbf{k}} \cdot \mathbf{e}\right|^{2} p_{i \mathbf{k}^{\prime} \mathbf{k}}\left\{A\left[\left.\rho_{i \mathbf{k}^{\prime} \mathbf{k}^{\prime}}\right|_{2}+\left.\rho_{i \mathbf{k} \mathbf{k}}^{12}\right|_{2}\right]+\left.\delta \Delta_{i}^{\prime}\right|_{2}\left(-\frac{\epsilon_{i \mathbf{k}^{\prime}}}{E_{i \mathbf{k}}} F_{i \mathbf{k} \mathbf{k}^{\prime}}^{12}-\frac{\epsilon_{i \mathbf{k}}}{E_{i \mathbf{k}}} F_{i \mathbf{k} \mathbf{k}^{\prime}}^{21}\right)\right\}\right\rangle_{\mathrm{Av}}
\end{aligned}
$$

Now we make the final approximation

$$
\left.\left\langle\left|\mathbf{J}_{i \mathbf{k}^{\prime} \mathbf{k}} \cdot \mathbf{e}\right|^{2} \rho_{i \mathbf{k} \mathbf{k}^{\prime}}^{a b}\right\rangle_{2}\right\rangle\left._{\mathrm{Av}} \approx\left\langle\left|\mathbf{J}_{i \mathbf{k}^{\prime} \mathbf{k}} \cdot \mathbf{e}\right|^{2}\right\rangle_{\mathrm{Av}}\left\langle\rho_{i \mathbf{k} \mathbf{k}^{\prime}}^{a b}\right\rangle_{\mathrm{Av}}\right|_{2} .
$$

With this, the differential equation becomes

$$
\begin{aligned}
{\left[i \partial_{t}-\left(E^{\prime}-E\right)\right] \frac{\left.\left\langle\mathbf{e} \cdot \mathbf{J}_{i \mathbf{k} \mathbf{k}^{\prime}} \rho_{i \mathbf{k} \mathbf{k}^{\prime}}^{11}\right\rangle_{\mathrm{Av}}\right|_{3}}{\left\langle\left|\mathbf{J}_{i \mathbf{k} \mathbf{k}^{\prime}} \cdot \mathbf{e}\right|^{2}\right\rangle_{\mathrm{Av}}}=} & l_{i}\left(\epsilon^{\prime}, \epsilon\right)\left\{A\left[\left.\left\langle\rho_{i \mathbf{k}^{\prime} \mathbf{k}^{\prime}}^{11}\right\rangle_{\mathrm{Av}}\right|_{2}-\left.\left\langle\rho_{i \mathbf{k k} \mathbf{k}}^{11}\right\rangle_{\mathrm{Av}}\right|_{2}\right]+\left.\delta \Delta_{i}^{\prime}\right|_{2}\left(\frac{\Delta_{i}}{E^{\prime}}-\frac{\Delta_{i}}{E}\right) F_{i}\left(\epsilon, \epsilon^{\prime}\right)^{11}\right\} \\
& +p_{i}\left(\epsilon^{\prime}, \epsilon\right)\left\{A\left[\left.\left\langle\rho_{i \mathbf{k}^{\prime} \mathbf{k}^{\prime}}^{21}\right\rangle_{\mathrm{Av}}\right|_{2}+\left.\left\langle\rho_{i \mathbf{k k}}^{12}\right\rangle_{\mathrm{Av}}\right|_{2}\right]+\left.\delta \Delta_{i}^{\prime}\right|_{2}\left(-\frac{\epsilon^{\prime}}{E^{\prime}} F_{i}\left(\epsilon, \epsilon^{\prime}\right)^{12}-\frac{\epsilon}{E} F_{i}\left(\epsilon, \epsilon^{\prime}\right)^{21}\right)\right\} .
\end{aligned}
$$

Note that $\left\langle\left|\mathbf{J}_{i \mathbf{k}^{\prime} \mathbf{k}} \cdot \mathbf{e}\right|^{2}\right\rangle_{\mathrm{Av}}=\left\langle\left|\mathbf{J}_{i \mathbf{k} \mathbf{k}^{\prime}} \cdot \mathbf{e}\right|^{2}\right\rangle_{\mathrm{Av}}$. Defining

$$
R_{i}^{a b}\left(\epsilon, \epsilon^{\prime}\right)=\left.\frac{1}{\left\langle\left|\mathbf{J}_{i \mathbf{k} \mathbf{k}^{\prime}} \cdot \mathbf{e}\right|^{2}\right\rangle_{\mathrm{Av}}}\left\langle\mathbf{e} \cdot \mathbf{J}_{i \mathbf{k} \mathbf{k}^{\prime}} \rho_{i \mathbf{k} \mathbf{k}^{\prime}}^{a b}\right\rangle_{\mathrm{Av}}\right|_{3},
$$

and noting

$$
r_{i}^{a b}(\epsilon)=\left.\left\langle\rho_{i \mathbf{k k}}^{a b}\right\rangle_{\mathrm{Av}}\right|_{2},
$$

we rewrite this as

$$
\begin{aligned}
{\left.\left[i \partial_{t}-\left(E^{\prime}-E\right)\right] R_{i}^{11}\left(\epsilon, \epsilon^{\prime}\right)\right|_{3}=} & l_{i}\left(\epsilon^{\prime}, \epsilon\right)\left\{A\left[r_{i}^{11}\left(\epsilon^{\prime}\right)-r_{i}^{11}(\epsilon)\right]+\left.\delta \Delta_{i}^{\prime}\right|_{2}\left(\frac{\Delta_{i}}{E^{\prime}}-\frac{\Delta_{i}}{E}\right) F_{i}^{11}\left(\epsilon, \epsilon^{\prime}\right)\right\} \\
& +p_{i}\left(\epsilon^{\prime}, \epsilon\right)\left\{A\left[r_{i}^{21}\left(\epsilon^{\prime}\right)+r_{i}^{12}(\epsilon)\right]+\left.\delta \Delta_{i}^{\prime}\right|_{2}\left(-\frac{\epsilon^{\prime}}{E^{\prime}} F_{i}^{12}\left(\epsilon, \epsilon^{\prime}\right)-\frac{\epsilon}{E} F_{i}^{21}\left(\epsilon, \epsilon^{\prime}\right)\right)\right\}
\end{aligned}
$$

which precisely gives Eq. 48 in [20]. Similar equations can be derived for the remaining $R_{i}^{a b}$. 
Rewriting the expression for the paramagnetic current $\mathbf{j}_{P}$ in terms of the $R_{i}^{a b}\left(\epsilon, \epsilon^{\prime}\right)$, and then making the Mattis-Bardeen impurity replacement (5), yields

$$
\left.\mathbf{j}_{P}\right|_{3}=\mathbf{e} \sum_{i} \frac{e^{2} n_{i}}{2 m_{i}} \int d \epsilon d \epsilon^{\prime} W_{i}\left(\epsilon, \epsilon^{\prime}\right)\left[l_{i}\left(\epsilon, \epsilon^{\prime}\right)\left(R_{i}^{11}\left(\epsilon, \epsilon^{\prime}\right)+R_{i}^{22}\left(\epsilon, \epsilon^{\prime}\right)\right)+p_{i}\left(\epsilon, \epsilon^{\prime}\right)\left(R_{i}^{21}\left(\epsilon, \epsilon^{\prime}\right)-R_{i}^{22}\left(\epsilon, \epsilon^{\prime}\right)\right)\right] .
$$

[1] C. M. Varma, Higgs Boson in superconductors, J. Low Temp. Phys. 126, 901 (2002).

[2] D. Pekker and C. Varma, Amplitude/Higgs modes in condensed matter physics, Annu. Rev. Condens. Matter Phys. 6, 269 (2015).

[3] P. W. Anderson, Random-phase approximation in the theory of superconductivity, Phys. Rev. 112, 1900 (1958).

[4] A. J. Leggett, Number-phase fluctuations in two-band superconductors, Prog. Theor. Phys. 36, 901 (1966).

[5] T. Papenkort, V. M. Axt, and T. Kuhn, Coherent dynamics and pump-probe spectra of BCS superconductors, Phys. Rev. B 76, 224522 (2007).

[6] H. Krull, D. Manske, G. S. Uhrig, and A. P. Schnyder, Signatures of nonadiabatic BCS state dynamics in pump-probe conductivity, Phys. Rev. B 90, 014515 (2014).

[7] N. Tsuji and H. Aoki, Theory of Anderson pseudospin resonance with Higgs mode in superconductors, Phys. Rev. B 92, 064508 (2015).

[8] H. Krull, N. Bittner, G. S. Uhrig, D. Manske, and A. P. Schnyder, Coupling of Higgs and Leggett modes in nonequilibrium superconductors, Nat. Commun. 7, 11921 (2016).

[9] A. Kumar and A. F. Kemper, Higgs oscillations in time-resolved optical conductivity, Phys. Rev. B 100, 174515 (2019).

[10] L. Schwarz, B. Fauseweh, N. Tsuji, N. Cheng, N. Bittner, H. Krull, M. Berciu, G. S. Uhrig, A. P. Schnyder, S. Kaiser, and D. Manske, Classification and characterization of nonequilibrium Higgs modes in unconventional superconductors, Nat. Commun. 11, 287 (2020).

[11] R. Matsunaga, Y. I. Hamada, K. Makise, Y. Uzawa, H. Terai, Z. Wang, and R. Shimano, Higgs Amplitude Mode in the BCS Superconductors $\mathrm{Nb}_{1-x} \mathrm{Ti}_{x} \mathbf{N}$ Induced by Terahertz Pulse Excitation, Phys. Rev. Lett. 111, 057002 (2013).

[12] R. Matsunaga, N. Tsuji, H. Fujita, A. Sugioka, K. Makise, Y. Uzawa, H. Terai, Z. Wang, H. Aoki, and R. Shimano, Lightinduced collective pseudospin precession resonating with Higgs mode in a superconductor, Science 345, 1145 (2014).

[13] K. Katsumi, N. Tsuji, Y. I. Hamada, R. Matsunaga, J. Schneeloch, R. D. Zhong, G. D. Gu, H. Aoki, Y. Gallais, and R. Shimano, Higgs Mode in the $d$-Wave Superconductor $\mathrm{Bi}_{2} \mathrm{Sr}_{2} \mathrm{CaCu}_{2} \mathrm{O}_{8+x}$ Driven by an Intense Terahertz Pulse, Phys. Rev. Lett. 120, 117001 (2018).

[14] F. Giorgianni, T. Cea, C. Vicario, C. P. Hauri, W. K. Withanage, $\mathrm{X}$. Xi, and L. Benfatto, Leggett mode controlled by light pulses, Nat. Phys. 15, 341 (2019).

[15] H. Chu, M.-J. Kim, K. Katsumi, S. Kovalev, R. D. Dawson, L. Schwarz, N. Yoshikawa, G. Kim, D. Putzky, Z. Z. Li et al., Phase-resolved Higgs response in superconducting cuprates, Nat. Commun. 11, 1793 (2020).

[16] K. Katsumi, Z. Z. Li, H. Raffy, Y. Gallais, and R. Shimano, Superconducting fluctuations probed by the Higgs mode in
$\mathrm{Bi}_{2} \mathrm{Sr}_{2} \mathrm{CaCu}_{2} \mathrm{O}_{8+x}$ thin films, Phys. Rev. B 102, 054510 (2020).

[17] S. Kovalev, T. Dong, L.-Y. Shi, C. Reinhoffer, T.-Q. Xu, H.-Z. Wang, Y. Wang, Z.-Z. Gan, S. Germanskiy, J.-C. Deinert, et al., Band-selective third-harmonic generation in superconducting $\mathrm{MgB}_{2}$ : Evidence for Higgs amplitude mode in the dirty limit, arXiv:2010.05019.

[18] C. Vaswani, J. Kang, M. Mootz, L. Luo, X. Yang, C. Sundahl, D. Cheng, C. Huang, R. Kim, et al., Light quantum control of persisting Higgs modes in iron-based superconductors, Nat. Commun. 12, 258 (2021).

[19] T. Cea, C. Castellani, and L. Benfatto, Nonlinear optical effects and third-harmonic generation in superconductors: Cooper pairs versus higgs mode contribution, Phys. Rev. B 93, 180507(R) (2016).

[20] Y. Murotani and R. Shimano, Nonlinear optical response of collective modes in multiband superconductors assisted by nonmagnetic impurities, Phys. Rev. B 99, 224510 (2019).

[21] M. Silaev, Nonlinear electromagnetic response and Higgs-mode excitation in BCS superconductors with impurities, Phys. Rev. B 99, 224511 (2019).

[22] N. Tsuji and Y. Nomura, Higgs-mode resonance in third harmonic generation in $\mathrm{NbN}$ superconductors: Multiband electronphonon coupling, impurity scattering, and polarization-angle dependence, Phys. Rev. Research 2, 043029 (2020).

[23] G. Seibold, M. Udina, C. Castellani, and L. Benfatto, Third harmonic generation from collective modes in disordered superconductors, Phys. Rev. B 103, 014512 (2021).

[24] D. C. Mattis and J. Bardeen, Theory of the anomalous skin effect in normal and superconducting metals, Phys. Rev. 111, 412 (1958).

[25] G. Seibold, L. Benfatto, and C. Castellani, Application of the mattis-bardeen theory in strongly disordered superconductors, Phys. Rev. B 96, 144507 (2017).

[26] H. Suhl, B. T. Matthias, and L. R. Walker, Bardeen-CooperSchrieffer Theory of Superconductivity in the Case of Overlapping Bands, Phys. Rev. Lett. 3, 552 (1959).

[27] A. Van Otterlo, D. S. Golubev, A. D. Zaikin, and G. Blatter, Dynamics and effective actions of BCS superconductors, Eur. Phys. J. B 10, 131 (1999).

[28] S. G. Sharapov, V. P. Gusynin, and H. Beck, Effective action approach to the Leggett's mode in two-band superconductors, Eur. Phys. J. B 30, 45 (2002).

[29] L. Benfatto, A. Toschi, and S. Caprara, Low-energy phase-only action in a superconductor: A comparison with the $\mathrm{XY}$ model, Phys. Rev. B 69, 184510 (2004).

[30] T. Cea, P. Barone, C. Castellani, and L. Benfatto, Polarization dependence of the third-harmonic generation in multiband superconductors, Phys. Rev. B 97, 094516 (2018). 
[31] T. Cea and L. Benfatto, Signature of the Leggett mode in the $A_{1 g}$ Raman response: From $\mathrm{MgB}_{2}$ to iron-based superconductors, Phys. Rev. B 94, 064512 (2016).

[32] K. R. Babu and G.-Y. Guo, Electron-phonon coupling, superconductivity, and nontrivial band topology in $\mathrm{NbN}$ polytypes, Phys. Rev. B 99, 104508 (2019).

[33] W. Zimmermann, E. H. Brandt, M. Bauer, E. Seider, and L. Genzel, Optical conductivity of BCS superconductors with arbitrary purity, Physica C 183, 99 (1991).

[34] E. A. Yuzbashyan and M. Dzero, Dynamical Vanishing of the Order Parameter in a Fermionic Condensate, Phys. Rev. Lett. 96, 230404 (2006).

[35] M. Udina, T. Cea, and L. Benfatto, Theory of coherentoscillations generation in terahertz pump-probe spectroscopy: From phonons to electronic collective modes, Phys. Rev. B 100, 165131 (2019).

[36] R. Matsunaga and R. Shimano, Nonequilibrium BCS State Dynamics Induced by Intense Terahertz Pulses in a Superconducting NbN Film, Phys. Rev. Lett. 109, 187002 (2012).

[37] J. Kortus, I. I. Mazin, K. D. Belashchenko, V. P. Antropov, and L. L. Boyer, Superconductivity of Metallic Boron in $\mathrm{MgB}_{2}$, Phys. Rev. Lett. 86, 4656 (2001).

[38] F. Giubileo, D. Roditchev, W. Sacks, R. Lamy, D. X. Thanh, J. Klein, S. Miraglia, D. Fruchart, J. Marcus, and P. Monod, Two-Gap State Density in $\mathrm{MgB}_{2}$ : A True Bulk Property or a Proximity Effect? Phys. Rev. Lett. 87, 177008 (2001).

[39] M. Iavarone, G. Karapetrov, A. E. Koshelev, W. K. Kwok, G. W. Crabtree, D. G. Hinks, W. N. Kang, E.-M. Choi, H. J. Kim, H.-J. Kim, and S. I. Lee, Two-Band Superconductivity in $\mathrm{MgB}_{2}$, Phys. Rev. Lett. 89, 187002 (2002).

[40] S. Tsuda, T. Yokoya, Y. Takano, H. Kito, A. Matsushita, F. Yin, J. Itoh, H. Harima, and S. Shin, Definitive Experimental Evidence for Two-Band Superconductivity in $\mathrm{MgB}_{2}$, Phys. Rev. Lett. 91, 127001 (2003).
[41] R. A. Kaindl, M. A. Carnahan, J. Orenstein, D. S. Chemla, H. M. Christen, H.-Y. Zhai, M. Paranthaman, and D. H. Lowndes, Far-Infrared Optical Conductivity Gap in Superconducting $\mathrm{MgB}_{2}$ Films, Phys. Rev. Lett. 88, 027003 (2001).

[42] Y. Murotani, N. Tsuji, and H. Aoki, Theory of light-induced resonances with collective Higgs and Leggett modes in multiband superconductors, Phys. Rev. B 95, 104503 (2017).

[43] G. Blumberg, A. Mialitsin, B. S. Dennis, M. V. Klein, N. D. Zhigadlo, and J. Karpinski, Observation of Leggett's Collective Mode in a Multiband $\mathrm{MgB}_{2}$ Superconductor, Phys. Rev. Lett. 99, 227002 (2007).

[44] N. M. Rugheimer, A. Lehoczky, and C. V. Briscoe, Microwave transmission- and reflection-coefficient ratios of thin superconducting films, Phys. Rev. 154, 414 (1967).

[45] E. F. C. Driessen, P. C. J. J. Coumou, R. R. Tromp, P. J. de Visser, and T. M. Klapwijk, Strongly Disordered TiN and NbTiN $s$-Wave Superconductors Probed by Microwave Electrodynamics, Phys. Rev. Lett. 109, 107003 (2012).

[46] P. C. J. J. Coumou, E. F. C. Driessen, J. Bueno, C. Chapelier, and T. M. Klapwijk, Electrodynamic response and local tunneling spectroscopy of strongly disordered superconducting tin films, Phys. Rev. B 88, 180505(R) (2013).

[47] A. Moor, A. F. Volkov, and K. B. Efetov, Amplitude Higgs Mode and Admittance in Superconductors with a Moving Condensate, Phys. Rev. Lett. 118, 047001 (2017).

[48] M. Puviani, L. Schwarz, X.-X. Zhang, S. Kaiser, and D. Manske, Current-assisted Raman activation of the Higgs mode in superconductors, Phys. Rev. B 101, 220507(R) (2020).

[49] I. J. R. Aitchison, P. Ao, D. J. Thouless, and X.-M. Zhu, Effective Lagrangians for BCS superconductors at $t=0$, Phys. Rev. B 51, 6531 (1995).

[50] EverettYou/MatsubaraSum: A Mathematica package to perform Matsubara summation analytically, available at https://github. com/EverettYou/MatsubaraSum. 